\title{
Review \\ The Forerunners on Heritage Stones Investigation: Historical Synthesis and Evolution
}

\author{
David M. Freire-Lista ${ }^{1,2}$ (D) \\ 1 Departamento de Geologia, Escola de Ciências da Vida e do Ambiente, \\ Universidade de Trás-os-Montes e Alto Douro, 5001-801 Vila Real, Portugal; davidfreire@utad.pt \\ 2 Centro de Geociências, Universidade de Coimbra, 3030-790 Coimbra, Portugal
}

Citation: Freire-Lista, D.M. The Forerunners on Heritage Stones Investigation: Historical Synthesis and Evolution. Heritage 2021, 4, 1228-1268. https://doi.org/10.3390/ heritage 4030068

Academic Editors: Carlos Alves,

Carlos Figueiredo and

Jorge Sanjurjo-Sánchez

Received: 11 May 2021

Accepted: 1 July 2021

Published: 12 July 2021

Publisher's Note: MDPI stays neutral with regard to jurisdictional claims in published maps and institutional affiliations.

Copyright: (C) 2021 by the author. Licensee MDPI, Basel, Switzerland. This article is an open access article distributed under the terms and conditions of the Creative Commons Attribution (CC BY) license (https:// creativecommons.org/licenses/by/ $4.0 /)$.

\begin{abstract}
Human activity has required, since its origins, stones as raw material for carving, construction and rock art. The study, exploration, use and maintenance of building stones is a global phenomenon that has evolved from the first shelters, manufacture of lithic tools, to the construction of houses, infrastructures and monuments. Druids, philosophers, clergymen, quarrymen, master builders, naturalists, travelers, architects, archaeologists, physicists, chemists, curators, restorers, museologists, engineers and geologists, among other professionals, have worked with stones and they have produced the current knowledge in heritage stones. They are stones that have special significance in human culture. In this way, the connotation of heritage in stones has been acquired over the time. That is, the stones at the time of their historical use were simply stones used for a certain purpose. Therefore, the concept of heritage stone is broad, with cultural, historic, artistic, architectural, and scientific implications. A historical synthesis is presented of the main events that marked the use of stones from prehistory, through ancient history, medieval times, and to the modern period. In addition, the main authors who have written about stones are surveyed from Ancient Roman times to the middle of the twentieth century. Subtle properties of stones have been discovered and exploited by artists and artisans long before rigorous science took notice of them and explained them.
\end{abstract}

Keywords: archeology; geology; fortification; durability; conservation; quarries; restoration; architecture

\section{Introduction}

Stones have been the subject of analysis since humans have sought the best shelters such as escarpments, caves and inverted slopes to protect themselves from inclement weather [1]. Therefore, mankind has explored and exploited building stones throughout history [2-4]. The best-preserved records of human activity are those carved on this material. Much of the current knowledge of the oldest civilizations is due to the study of their heritage stones. The stones that today are considered heritage stones were used by humans mainly as building stones and sometimes they were used for purposes other than those they have today.

The importance of stones is evidenced by the pre-Roman roots Mor (r), Mur (r), Mour present in numerous place names of Iberian Peninsula. For example, La Moraleja, Matamoros, Moralzarzal, Moratalla, Montemuro, Moreiras, Morro, Mouçós, Moura, Mourelle, Murgi and Valdemoro, are names of localities linked to stone; material with which muros "walls" and moradas "dwellings" have been built since prehistoric times. The first stones used as construction material came from the vicinity of the place where they would be used. They were used as prehistoric tools [5,6], amulets [7], support for petroglyphs [8,9], projectiles [10], support for inscriptions of important events or laws [11], walls [12], monuments, dikes, warehouses, paving [13] and all kinds of constructions [14]. Stones have also been used to manufacture mortars [15-17], ceramics [18,19], bricks [20,21] and mosaics [22].

Many ancient cities were built directly on quarries, resulting in harmony in between the landscape and the inhabited environment $[23,24]$. Exploitation of stones generated large 
quarries from which they were extracted for export [25-27]. Some stones were transported long distances and traded for their rarity and durability, [28,29] and some prehistoric quarries have survived to this day.

The study of building stones has evolved from a practical way to make ornaments, tools, houses, temples and infrastructures, among many other constructions to being the subject of scientific research as we know it today. The study of heritage stones cannot be understood without the contribution of druids, clergymen, quarrymen, builders, naturalists, architects, archaeologists, physicists, chemists, curators, restorers, engineers, and geologists, who have all contributed to enriching our current knowledge of heritage stones. Which have been the subject of exposure and study since the first museums started to conserve them, such as the Ennigaldi-Nanna museum in Mesopotamia, dating from 530 BC [30].

Some examples of heritage stones have a special relevance in human culture. For example, the porphyries from Sweden and Russia [31,32], "Petit granit" and Lede stones from Belgium [33,34], slates from the Iberian peninsula [35], Bath and Purbeck limestones from England [36], Sydney sandstone [37], granites such as Piedra Berroqueña, of Madrid (Spain) [38-40] and marbles, such as the one from Carrara in Italy [41,42]. These stones have been widely used and with them important buildings have been built that have marked the history of humankind [43].

This work is a historical synopsis of the main events that marked the history of the use of heritage stones. It contains references of the main authors who have studied stones in the ages of human history, focusing on southern European countries, and without missing the important development that research has had in the United States from the end of the 19 th century to the 20th century. Scientists from a wide variety of disciplines contributed to the current understanding of heritage stones, and the most important are mentioned in this work.

\section{Stones and Religion}

Many myths compare stones to the "bones" of Mother Earth. Stone extraction has been accompanied by complex rituals in different cultures [44]. Stones have also been used in healing therapies and in numerous festivities and pilgrimages. The Holy Black Stone (called al-Hayar-ul-Aswad in Arabic) is a relic that according to Islamic tradition dates to the times of Adam (Adam) and Hawa (Eve). It is a meteorite that the archangel Gabriel (Jibril) gave to Abraham (Ibrahim), who, together with his son Ishmael (Ismail), placed it in the eastern corner of the Kaaba, in the center of the Great Mosque of Mecca, in Saudi Arabia [45-47]. Pedro (Petro- $\pi \dot{\varepsilon} \tau \rho o \varsigma)$ is the masculinization of the Greek word $\pi \dot{\varepsilon} \tau \rho \alpha$ (Petra), that is to say "stone". Paul the Apostle always called Peter by the name "Cephas", a Hellenized Hebrew word from the Aramaic N'כ, meaning "stone" and nowadays many stonemason villages keep their patronal festivals in honor of Saint Peter.

Jade objects and amulets, symbolizing nobility, perfection, constancy, and immortality have been very valued in Chinese culture [48,49]. Jade was also used to make weapons and tools due to its resistance and hardness. Jade was the stone of creation; it meant life, fertility and power for pre-Hispanic cultures of Mesoamerica such as the Olmec, Mayan, Toltec, Quiche, Mixtec, Zapotec, Aztec (Chalchiúhuitl) and Nicoya. It was obtained mainly from the Motagua Valley (Guatemala). This region has been the source of the jade used by Mesoamericans for thousands of years [50].

Quartz has been used as an amulet, acquiring a sacred character in Neolithic, possibly due to its resistance to weathering, frequent transparency and chromatic homogeneity. As well as due to its crystallization in the hexagonal and trigonal system, being able to form hexagonal bipyramids [51]. Quartz has also been a mineral used to create fire. There are cultures in which the word quartz retains a meaning related to the sun. The Irish word for quartz is grian cloch, which means "stone of the sun" An example of the use of flint as a solar baetylus (sacred stone) is found in the southern temple of Mnajdra (Malta) [52-55]. It is one of the oldest free-standing structures in the world (approximately $3000 \mathrm{BC}$ ), even predating the pyramids of Egypt and Stonehenge. The entire temple is built with limestone, 
except for an ashlar located on the threshold of the main door, where there is a vein of flint (Figure 1). The intersection point of the dawn light beams of the equinox and summer and winter solstices coincides with the center of the vein of flint at the threshold of the main gate of the southern temple of Mnajdra. Using flint as a baetylus of solar character.

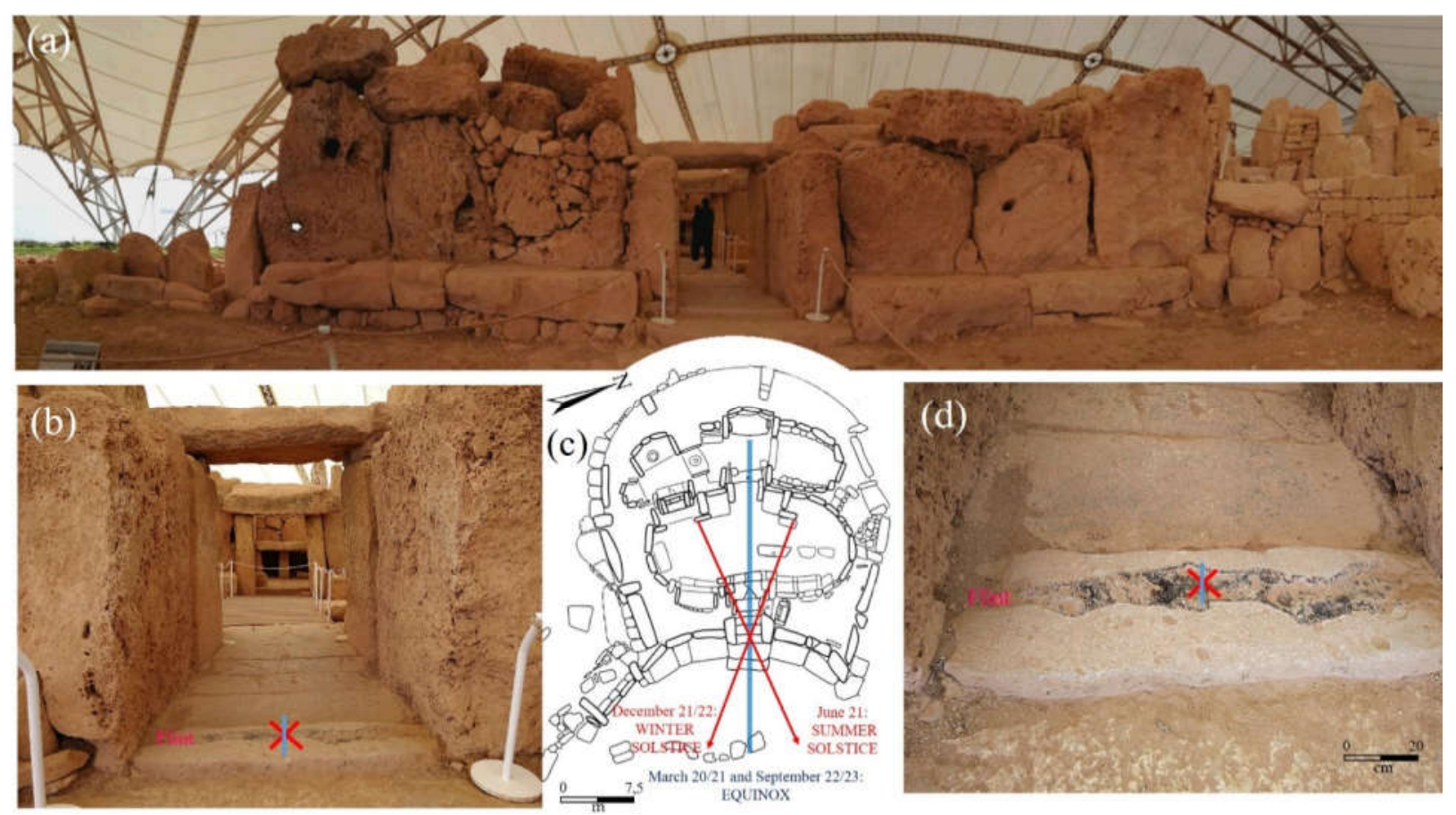

Figure 1. South temple of Mnajdra (Malta). (a) General view of the main façade. (b) Main door. Note that the threshold has a vein of flint. (c) Plan of the temple with the projection of the light beams corresponding to the equinox (blue) and the summer and winter solstices (red). Note that the intersection point corresponds to the threshold with the flint vein. Modified from J.D. Evans, The Prehistoric Antiquities of the Maltese Islands: A Survey, University of London, Athlone Press, 1971. (d) Threshold of the main door with presence of a vein of flint.

Prehistoric tools were made with basalt, diabase, andesite, quartzites, flint, quartz, obsidian and other stones [56]. These tools (Figure 2) were inseparable companions in the daily life of their manufacturers, even after death. Stone axes and arrowheads have been found attached as amulets in necklaces excavated from Egyptian and Etruscan tombs, and from Chaldean temples. Animistic religions associated these objects with spirits or gods, and attributed powers related to atmospheric phenomena and divinities to them. For example, the fall of lightning created stone axes that were called "astropelekia" (sky axes) in Greece [57]. Prehistoric axes (Figure 2), polished or not, were called "cerauni" by Romans and "lightning stones", or simply "lightning bolts" in many Western European countries, for example "Arrows of God" in Hungary, "fairy darts" in Ireland, "thunder stone" in Iceland, "stones of Ukko" (god of lightning) in Finland, "arrows of thunder" in Siberia.
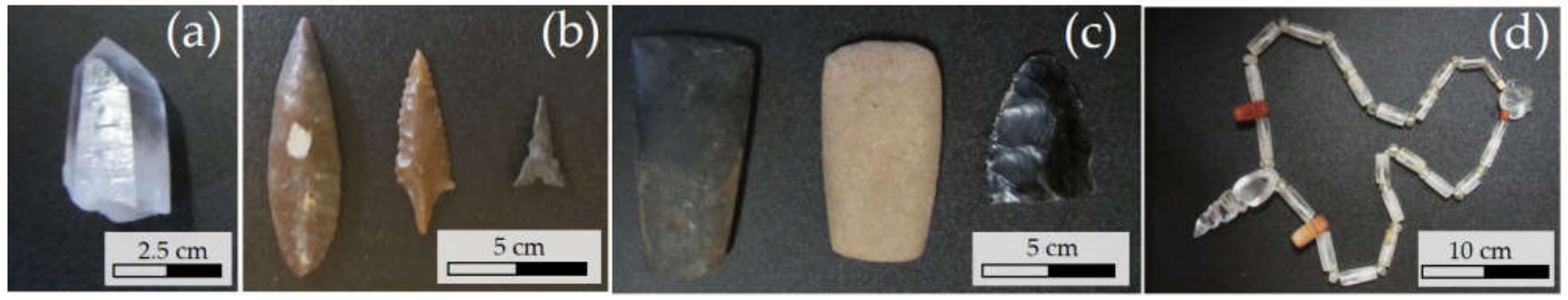

Figure 2. (a) Hyaline quartz baetylus found in a Neolithic site; (b) flint arrowheads; (c) axes of basalt, quartzite and obsidian; (d) necklace of hyaline quartz beads. 
Vikings saw the primitive tools as lightning repellent. Prehistoric "thunderstones" resembled the Norse god Thor's hammerhead (Mjöllnir) and they were purposely placed as good-luck talismans in tombs of Vikings. Additionally, a popular belief exists in some islands in Southeast Asia that prehistoric stone axes (and/or adzes) are natural objects generated by lightning. They are called "teeth of lightning" in Java and "arrows of lightning" in India or Japan. Elongated stones, the work of the first inhabitants of Borneo, were venerated and received the name "Silum Baling Go" (nail of the big toe of Baling Go) (god of thunder), because the natives believed that they had fallen from the sky. All of them are names that indicate the importance of stones in different civilizations since prehistory [58].

The Tuaregs of North Africa believed that polished axes were stones dropped from the sky, and that they had beneficial health properties. The importance of meteoric origin stones is evidenced by the presence of laminated metal composites in daggers and other ritual objects such as those found in the Great Pyramid of Giza and Tutankhamun's iron dagger blade $[59,60]$. Perhaps the earliest known reference to improved properties in a laminated metal composites can be found in The Iliad of Homer (800 BC) which describes Achilles' shield. In addition, Elagabalium temple (218-222 AC) was dedicated to the Sun god in the northeast corner of the Palatine Hill, in Rome (Italy). This temple had a baetylus at center, that is, Sol Invictus (Unconquered Sun) was represented by a conical black stone, which has been suggested to be a piece of a meteorite (Figure 3).

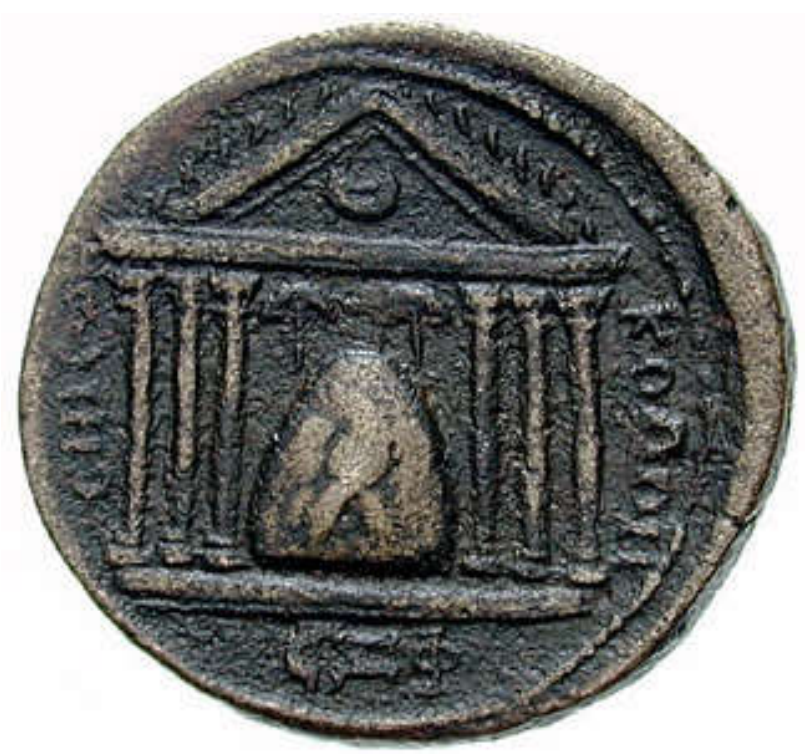

Figure 3. Roman coin of 3rd century AD. The Emesa temple to the sun god Elagabalus with a baetylus at center.

\section{Prehistory}

Humans soon discovered that microcrystalline and vitreous stones fragment conchoidally, producing cutting edges [61] (Figure 3). The Stone Age, or lithic stage, is the first period of prehistory that ranges from the time when human beings began to make tools and vessels with stones, to the discovery and use of pottery and metals [62,63].

The profound change that characterizes the outset of the Neolithic period affected human lives at various levels, e.g., subsistence, technology, environment, social organization, [64] and stones were part of all these levels. They were essential for the development of agriculture, as tools to plow the land and to hunt animals which were represented in painted caves. It is possible that the Neolithic cave painters suffered hypoxia and hallucinations due to lack of oxygen in deep caves [65], which endowed the caves with mystical properties. Pigments extracted from stones were also used for rock-art decoration [66,67].

Building stones specialized as building techniques became more sophisticated. For example, the use of domes or vaults required a suitable type of stone, usually lighter [68]. 
Khirokitia in Cyprus dates from (ca. 6800-ca. 5200 BC). The circular buildings of this archaeological site supported beehive-shaped corbel domed vaults of unfired mud-bricks and stones, and they also represent some of the earliest evidence of dwellings with an upper floor. Similar beehive-shaped tombs, called tholoi, exist in Crete and Northern Iraq. Cornelian necklaces (a variety of brown chalcedony) have also been found in Khirokitia, indicating exploration, trade, and transportation of this material $[69,70]$.

The first walled settlements of the Old Iron Age were areas surrounded by stone barriers driven into the ground and wide walls of adobe masonry. Vitrified walls were made with masonry and fired adobes [71-73] composed mainly of bones, rubble and earth that served to compact the dry-laid masonry from the late Bronze Age (7th-6th centuries $\mathrm{BC}$ ) to that of the late Iron Age (2nd century BC).

Stones from several kilometers far-away quarries were used (Figure 4) for the construction of megalithic structures. These early quarries had optimal cleat spacing and exfoliation microcracks for the extraction of blocks with the appropriate dimensions [74].
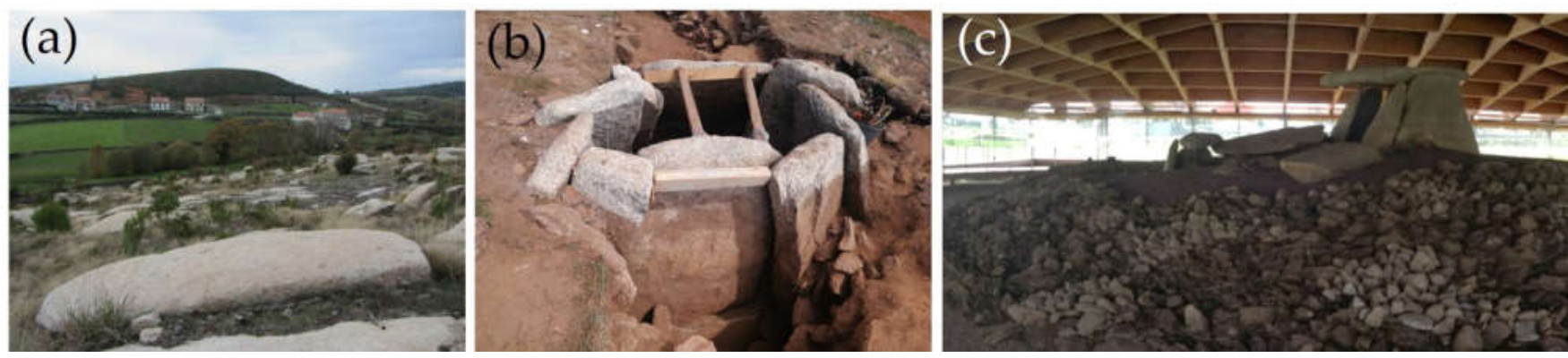

Figure 4. (a) Slab quarries of the Alto do Cotorino dolmen (northern Portugal); (b) Alto do Cotorino dolmen; (c) Dombate dolmen, Borneiro (northern Spain).

Walls of caissons, in a compartmentalized way, were built in modules in the 4th century BC $[75,76]$. As human activities became more specialized, construction materials had to meet the increasingly specialized needs and thus, for example, the most abrasive stones were used for the construction of mills. Stones that resisted high temperatures together with clays were used in smelting furnaces [77]. Humans also selected the material for firing ceramics $[78,79]$. This attests to a knowledge of the quality and properties of the stones.

Granite slabs with highly developed exfoliation microcracks.

\section{Ancient History}

\subsection{Mesopotamia}

Even though adobe was the main building material used in Mesopotamia, building stone exploration, cutting, carving and polishing techniques were developed in this area. In fact, the first mentions written about stones as eternal material, are in the Epic of Gilgamesh (ca. 2500-2000 BC). Land and river routes and the role of nomads were critical to understanding the use of heritage stones. Obsidian was used for arrowheads, blades, burins, and sickles. It came from Tell Mozan (Urkesh, Syria) and from other sites [80].

Syrian, Anatolian, and Mesopotamian cultures have interacted and mixed through history. The increased use of stone in southern Mesopotamia appears to be directly linked to the increasing significance of the temples. At different times, northern Mesopotamia and Anatolia, the Iranian Plateau, the Persian Gulf (Dilmun and Magan), and the distant Indus Valley (Melubba) were centers from which southern Mesopotamia obtained stone resources.

The first civilizations appeared in Sumeria [81] where adobe was commonly used for construction. The rocks were reserved for temples and palaces and in any case, walls. A lapis lazuli foundation tablet, preserved in the British museum, records that king LugalSilasi built the courtyard wall of a temple complex for the gods An and Inanna temple in 
Uruk [82]. Colonies of this city could be the agents responsible for the increased shipment of exotic stones into southern Mesopotamia. The temple of the Storm God was discovered beneath Aleppo's Ottoman citadel [83]. It was first constructed by Early Bronze Age peoples with a neat row of carved hard stone ashlars, with gods and mythical creatures. These ashlars have sharply chiseled surfaces show the marks of the sculptors.

An expansive variety of objects as beads, amulets, pendants, vessels, statuary and inlay were produced from amber (and other resins), agate, alabaster, aragonite, azurite, basalt, beryl (aquamarine, emerald), calcite, corundum (emery), quartz (citrine, crystal rock, smoky quartz), cornelian, chalcedony (agates, jasper), heliotrope, flint, onyx, ruby, sapphire, diorite, feldspar, garnet, granite, grindstones (nephrite, jadeite), gypsum, jet, lapis lazuli, limestone, malachite, marble, steatite, chlorite, serpentine, and turquoise among other stones. These objects were placed in temples as votive offerings. From the Late Uruk period to the end of the Early Dynastic period, light colored stones predominated in manufactured statuary, wall plaques, rare royal monuments, maceheads, and even cylinder seals. Toward the end of the Early Dynastic II and throughout the Akkadian and Ur III periods, there was a significant shift to dark stones: diorites, gabbros, and dolerites that were used principally for the manufacture of items for rulers and their high officials. These dark stones, no doubt expressive of the elite status of their owners are believed to have been sourced from the Persian Gulf, although it should be noted that they were also extensively found in the Makkran Range of south eastern Iran. The earlier lighter stones came from the nearby chains of the Zagros Mountains.

Serpentine was the most used stone to produce seals in the Late Uruk and Akkadian periods in southern Mesopotamia. In post-Akkadian and Ur III times, serpentine was replaced by chlorite to produce seals. Additionally, there were several workshops producing decorated vessels in chlorite/steatite coming from different sources. Darre Goodmordane Ashin and Sardare Noe Ashin were the main quarries. The outcrops of Konar Sandal and Tepe Yahya represented a significant stone workshop within the Iranian plateau [84].

Lapis lazuli is one of the first stones used for decorative artefacts and has been found in prehistoric tombs in Asia, Africa, and Europe. Lapis lazuli was imported to Mesopotamia in a semi-processed state from Afghanistan and, in less quantity, fom Pakistan [85]. The raw lumps of lapis lazuli recovered from fourth-millennium BC Djebel Aruda in Syria and fourth-millennium BC Mehrgarh in Pakistan indicated both the widespread and early use of this stone. It remained throughout the third millennium a highly prized stone. Throughout the recorded use of lapis lazuli, its principal source has been the Badakshan district of Afghanistan, which has been functioning as a quarry for approximately six thousand years and has been mentioned frequently in historical documents [86].

King Nabonidus (556-539 BC), of the Neo-Babylonian Empire (612-539 BC), discovered and analyzed the foundation deposits of the Akkadian Empire ruler Naram-Sin (ca. 2200 BC) in Ancient Mesopotamia. This king is thus known as the "first archaeologist" [87]. The Mesopotamians developed methods for cutting and polishing stones. Drill bits of diamond were first used in the Sassanian period (224-651 AC) and were procured from India.

\subsection{Ancient Egypt}

The Egyptians used copper tools with abrasive stones to cut rocks, a technique probably used first for quarrying stone blocks and later in excavating temple rooms inside rock mass. Slaves extracted large blocks of high-quality stones from quarries that were well organized $[88,89]$. Crushed stones and gypsum were also used to make plaster and mortar [90]. The sculptors chose the type of stone to carve according to the importance or durability they wanted to give to the finished work. In this way, statues of kings were carved mainly in igneous stones, and the bas-reliefs inside temples in softer stones such as sandstones and marl [91,92] (Figure 5). Some of the stone of the pyramids at Giza were quarried from the Giza Plateau. Other parts, such as casing stones, were quarried from the Tura area [93]. 

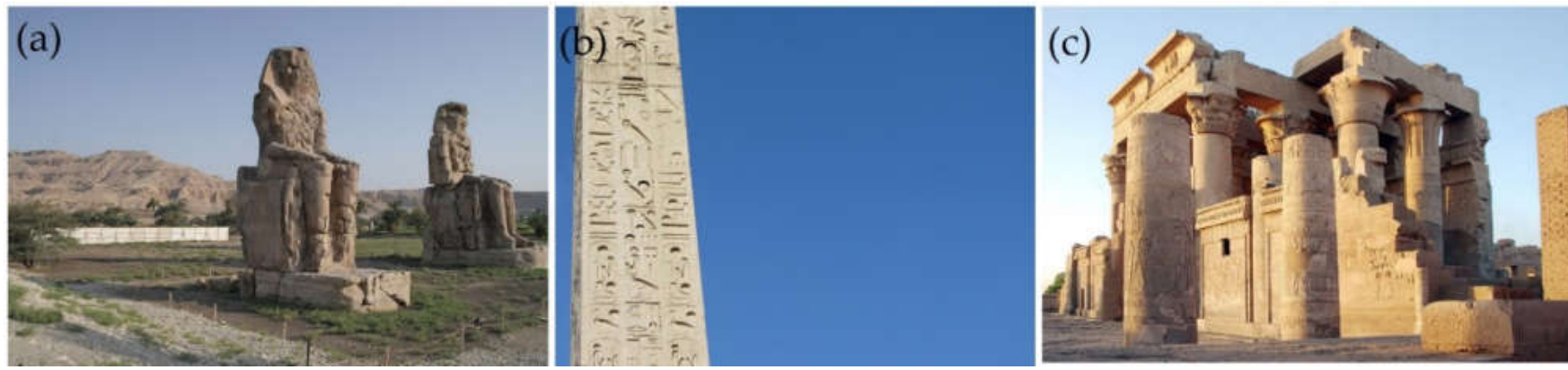

Figure 5. (a) Colossi of Memnon in front of Luxor city, near Medinet Habu (Egypt) carved in quartzite sandstone which was quarried at el-Gabal el-Ahmar (near modern-day Cairo) and transported 675 km. (b) Flamínio Obelisk, reign of Ramesses II and Merneptá (13th century BC) carved in granite. It moved to Rome by order of the Emperor Augustus and was placed in the spina of the Circus Maximus in 10 BC. It was abandoned and broken into three pieces after the fall of the Roman Empire and discovered in 1587. It was restored and erected in the Piazza del Popolo in 1589. (c) Kom-Ombo temple (Egypt) The construction of the temple with sandstone blocks was initiated by Ptolemy VI Philometor (180-145 BC).

Imhotep or Imutes (ca. 2690-ca. 2610 BC) was the "first known architect and engineer". He was the author of the funerary complex of Saqqara which has a wall of approximately $1500 \mathrm{~m}$ in perimeter, with various buildings and towards the center a stepped pyramid of six tiers with an approximate height of $60 \mathrm{~m}$. It was built with relatively small blocks of limestone, easy to transport and to handle. Previously, adobe bricks were used. The tomb of Djoser (Zoser) and galleries to store funerary vessels and clay figures were excavated under the pyramid [94]. A son of Ramesses II, Khaemweset (ca. 1281-ca. 1225 BC) is called "the first Egyptologist". He was interested in identifying and restoring the monuments of Egypt's past, such as Djoser's pyramid [95].

\section{Classical Antiquity}

Classical Antiquity comprises the Greco-Roman world, that is civilizations of ancient Greece and ancient Rome. These civilizations flourished and wielded great influence throughout Europe, North Africa and the Middle East.

\subsection{Classic Greece}

The extensive use of stones as building material by the ancient Greeks began during the 7th century BC. The raw materials were mostly marbles, travertines (" $\pi \omega \rho \rho \varsigma^{\prime \prime}$ ), sandstones, conglomerates, gneisses, schists, serpentinites, magmatic rocks, limestones and many others [96]. The ancient quarrymen selectively mined marbles of high quality and purity. The columns were extracted from quarries with few fractures or with joins that would favor extraction. Ancient Greek writers used various names for the quarries. Plato (ca. 427-ca. 347 BC), Strabo (64/63 BC-ca. 19/4 BC) and Plutarch (ca. 46-119 AC) called them " $\lambda \alpha \tau o \mu i \varepsilon \varsigma^{\prime}$ ", and Thucydides (ca. 460-ca. 400 BC), Herodotus (484-425 BC), Xenophon (ca. 431-354 BC), Theophrastus (ca. 431-354 BC) and Pausanias (480 BC-?)

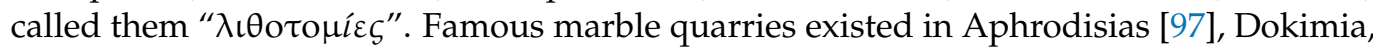
Doliana, Ephesos, Euboea, Hymettus, Naxos, Paros, Pentelikon, Prokonnesus (Marmara sea), Skyros and Thassos.

The Greeks had a better knowledge than Egyptians did of the mechanical and petrophysical properties of stones, and they built temples and sculptures with harmonious proportions. The first stone ashlar blocks of Greek architecture are those of the mid-7th century temples at Isthmia and Corinth. Their ashlars have grooves that were plausibly used to move the blocks with ropes. Forerunners of the crane appeared in Greece well before the late 6th century BC [98]. 
It is thought that the Greek philosopher Aristotle (384 BC-322 BC) described seven hundred stones giving their color, greatness, virtue and location [99], but the first preserved written references to the subject of building stones and their decay were made by the Greek philosopher Theophrastus (ca. 372 BC-287 BC), who described numerous stones used in classical Greece in Peri liton [100,101]. Theophrastus wrote about the regular use of mortar to cement stones in Cyprus and Phoenicia. Later, the geographer Strabo (ca. 64/63 BC-ca. 19/4 BC) made a description of the world as it was known in ancient times in Geography, making references to building stones. Claudius Ptolemy (ca. 100 AC-ca. $170 \mathrm{AC}$ ) wrote another work titled Geography, with a description of the known world, although with serious errors in distances. Ptolemaeus described, among other cities, Mecca, on the Arabian Peninsula.

\subsection{Classic Roman Empire}

The expansive Roman Empire and its taste for building stones of different colors [102,103] demanded a large supply of good quality stones (Figure 6). Paved roads were developed, and the most suitable stones for their elaboration were volcanic or philonian stones such as basalt, porphyry and diabase $[104,105]$, due to their low abrasion index. These communication routes allowed the transport of heritage stones to cities and villages $[106,107]$ to build walls, bridges, aqueducts, dams, stelae, sarcophagi, mosaics [108], mills [109] and ornamental elements [110]. Quarries were scattered throughout the Roman Empire [111-113] and a trade in ornamental stones was created, mostly from Egypt [114-116].
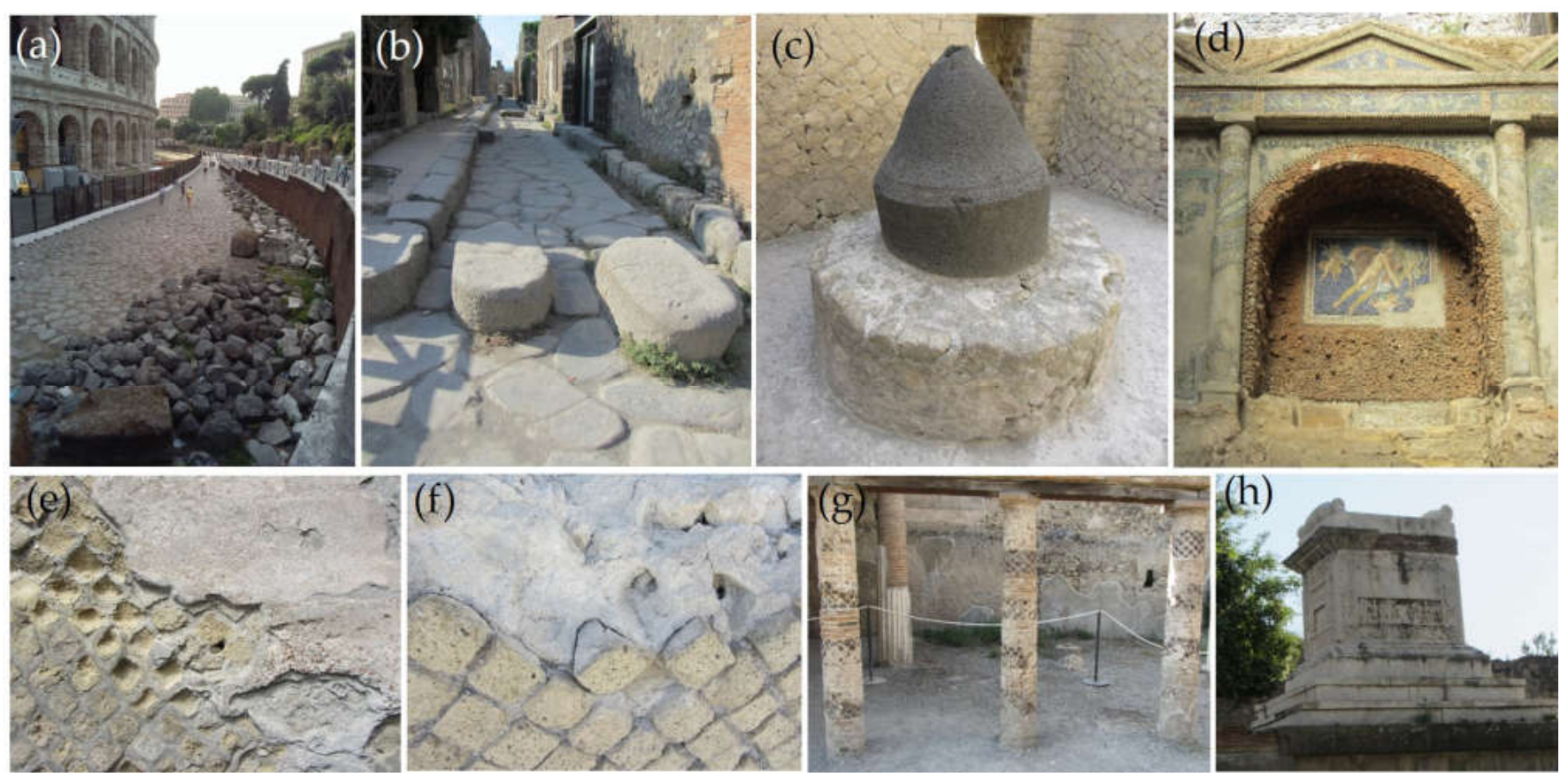

Figure 6. (a) Basalt pavement, Rome (Italy); (b) cobbled street with basalt, Pompeii (Italy); (c) basalt mill, Herculaneum (Italy); (d) nymphaeum with marble, pumice and other stones, Pompeii (Italy); (e) opus reticulatum, Herculaneum (Italy); (f) opus reticulatum, Herculaneum (Italy); (g) opus mixtum, Pompeii (Italy); (h) Pompeii marble tomb (Italy).

The Collegia Fabrorum were the groups of stonemasons, master builders and architects who obtained their knowledge about the cutting, carving and working of stones and their properties from Phoenician and Greek architects, who in turn had received it from the architects of Ancient Egypt. A Roman bas-relief of 3rd century AD shows a hydraulic mill to cut stones. It is possibly the first representation of this type of machine used to cut stones [117]. 
The stones were classified into four types according to their use: lapis vivus or franchus, hard stones for quality works and sculptures; lapis villanus, soft stone for lower quality constructions; lapis maceralis, stones for masonry and interior filling of the walls; and lapis columnarios, stones of great resistance for pillars [118]. Generally, white marble was used to make column capitals, bases and entablatures. Colored stones were used for column shafts, while both white and colored stones were used as wall veneer and floor paving. Small fragments of colored stones (called tesserae) were used to create mosaics.

The Roman mortars were a mixture of lime and a volcanic ash called pozzolana. Different materials were used as aggregates, primarily broken bricks and tiles and roughly fist-sized pieces of a volcanic stones called tufa. Concrete was mainly used to make foundations, walls and vaults. Concrete walls were faced with either brick or stones. Brick-faced concrete was called opus testaceum. Concrete faced with irregularly shaped stones was referred to as opus incertum. Opus reticulatum was made up of stone pieces in the shape of a quadrangular pyramid. The square faces were laid out, forming a diagonal grid (Figure 6). Walls with both brick and reticulate facing were called opus mixtum. Walls faced with alternating rows of bricks and small rectangular stone blocks were called opus vittatum.

The ancient Roman work that most closely resembles a scientific book was De rerum natura (On the nature of things) by Titus Lucretius Carus (ca. 99 BC-ca. 55 BC), a poet and philosopher, but this author hardly touches on geology and stones. The architect Marco Vitruvio Pollione ( $80 \mathrm{BC}-15 \mathrm{BC}$ ) described quarries and building materials, addressing their durability in De Architectura Libri Decem (The Ten Books of Architecture) [119]. Later, Gaius Plinius Secundus (Pliny the Elder) (23/24-79 AC) wrote the 37 books that make up Naturalis Historia (Natural History) based on what he witnessed: local people, animals, plants and stones. Pliny dealt with aspects related to building stones in the last three books. The book XXXV describes the uses of the land, pigments, sulfur and the art of painting. The book XXXVI describes lapidary, sculpture, architecture, obelisks, pyramids, Cretan labyrinths, clay, sand, stone, glass and the use of fire. Finally, the book XXXVII quotes extensively from a variety of literary sources concerning stones from all over the Roman Empire as rock crystal, amber, diamonds, gems and semi-precious stones [120].

Knowledge of building stones and carving techniques spread throughout the Mediterranean basin and Europe in recent prehistoric times and, especially, during the Iron Age. The walls of emplekton, two parallel faces with a core filled with finer material, had a high constructive quality (Figure $7 \mathrm{~b}$ ). For example, the fortified village of Castroeiro (Mondín de Basto, north Portugal) which was occupied from the 4th century BC to the first 1st century BC [121] conserves some very regular ashlars with a trapezoidal plan and pseudo-rectangular front (Figure 7), which certifies skill in carving granite ashlars.

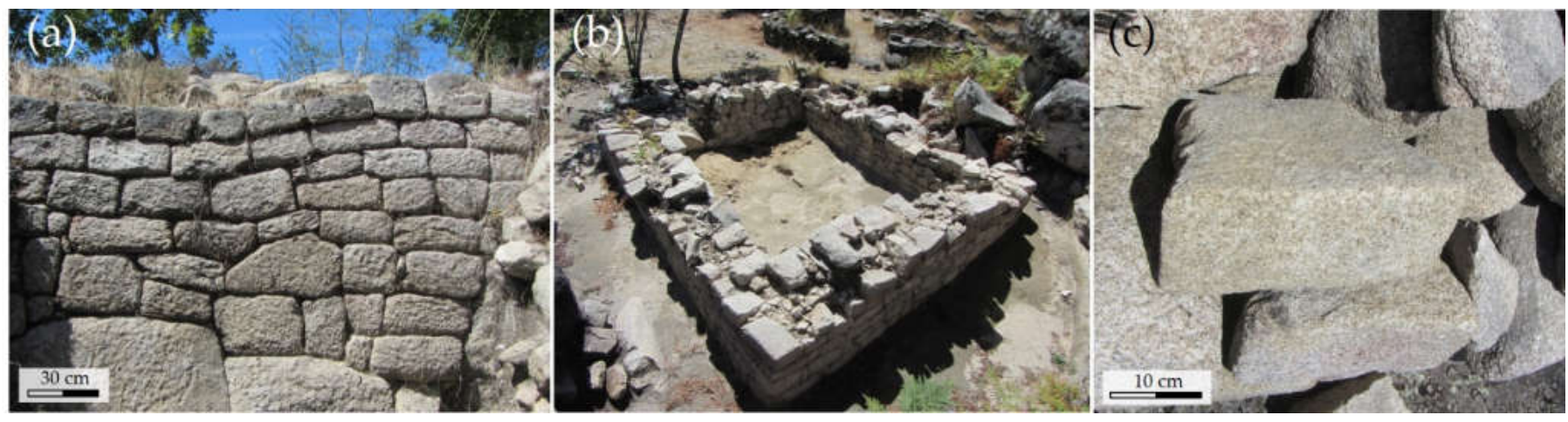

Figure 7. (a) Perimeter wall with granite ashlars carved in the shape of a trapezoidal prism; (b) wall of emplekton with granite ashlars in a house; (c) detail of granite ashlar carved in the shape of a trapezoidal prism. 


\section{Post-Classical History \\ 6.1. Middle Ages \\ 6.1.1. Early Middle Ages}

After the disappearance of the Roman Empire (476 AC), settlement patterns showed hill forts occupations and a new form of peasant site with the abandonment, dismantling or reorganization of Roman villas [122]. The idea of monasteries spread across the Byzantine Empire and then to Western Europe, where they adopted their own distinct practices based on the teachings of the Italian abbot Saint Benedict of Nursia (ca. 480-ca. 543 AC), regarded as the founder of the European monastery model. The reuse of Roman building stones was common and widespread in this period [123]. Many of Byzantine churches were attached to Roman temples and were built with reused Roman elements, such as the Saints Cosmas and Damien basilica, in the Roman Forum (Italy); the Santa Maria in Cosmedin basilica (or Schola Graeca), in Rome (Italy). The basilicas of Saint Apollinare the New and Saint Apollinare in Classe (Italy), the church of San Vital de Ravenna in Ravenna (Italy), Nostra Segnora de Mesumundu (Our Lady of Mesumundu) in Sardinia (Italy) and Chiesa di San Giovanni degli Eremiti (church of Saint John of the Hermits) in Palermo (Italy) are some examples of Byzantine churches built with bricks and they have austere façades. The stones, which were more expensive, were destined for more relevant buildings, such as the Mausoleum of Theodoric in Ravenna (Italy) (ca. 520 AC), built with white Istrian limestone (Croatia) [124]. However, in the Iberian Peninsula, especially in the north, and more western countries, building stones continued to be mainly used as a load bearing element [125].

The basilica of Hagia Sophia in Constantinople (Istanbul) 532-537 AC is the most representative building of this time. The marble used for the floor and ceiling was quarried from Anatolia (present-day eastern Turkey) and Syria, while bricks (used in the walls and parts of the floor) are from different construction phases with different clays composition [126]. The interior of Hagia Sophia is lined with enormous stone slabs sourced from multilple places: Green marble of Karystos (Greece), rose-colored marble from Phrygia (Turkey) [127], red Imperial porphyry from Egypt [128], Green porphyry from Sparta (Greece), buff lassikos from Caria (Turkey), white-yellowish marble from Lydia (Turkey), gold-colored marble from Libya, chunky black and white Celticum breccia (so-called marmum celticum) from France, honey-colored onyx from Pamukkale (Turkey), green Verde Antique serpentinite breccia from Thessaly (Greece) [129,130], white marble from Proconnesos (Turkey) [131] and the grey-colored marble from Vosporos (Greece) [132]. Hagia Sophia's columns were brought from the temple of Artemis in Ephesus (Turkey), from Egypt, and from other locations of the Byzantine Empire.

Starting in the 8th century, the Medieval Warm Period influenced the development of large numbers of quarries to build the great monasteries that exploited increasingly extensive land holdings and churches $[133,134]$ where stone was essential for construction. Ancient churches were rebuilt throughout Europe from the 9th century on to build great cathedrals that required the best available building materials.

In $793 \mathrm{AC}$, the Viking era began with the looting of the Lindisfarne monastery in north-eastern England [135,136]. Beginning with this event and until approximately 1100 AC, the Vikings looted monasteries, abbeys, and cities throughout Europe. Viking balances, weights and touchstones, tools to test the quality of non-ferrous metal, became widespread in the northern part of Europe as a sign of the buried individual's access to precious metals $[137,138]$. The cities were reinforced in anticipation of new attacks. Hard stones were used to reinforce city walls beginning in the 9th century, as a response to the development of counterbalanced powered war machines and Viking attacks. Thus, for example, coastal defensive towers were built, such in Catoria (Galicia, Spain) (Figure 8) and Santiago de Compostela was walled with stones [139]. 
Compositiones ad tingenda musiva, approximately from the 8th century, is the oldest surviving recipe book for sumptuary arts. It is not specifically dedicated to stones, although there are references to them. Similar in content to this recipe book is the one known as Mappae Clavicula, which must have circulated since the 9th century. This book contains the recipe of a glue for stones and for the manufacture of artificial stones.
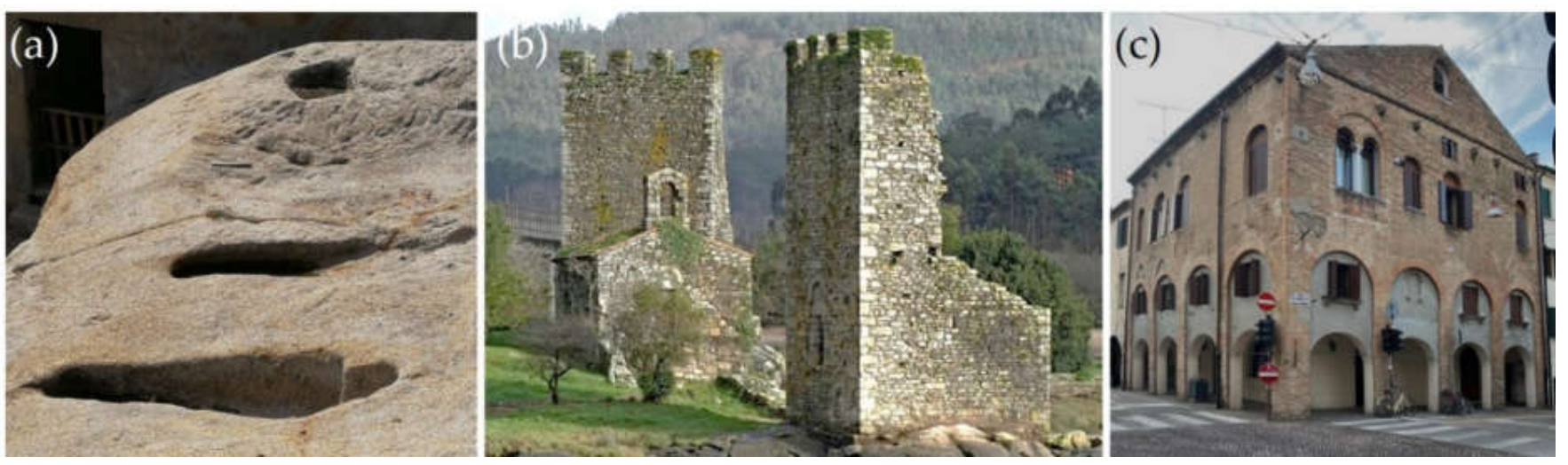

Figure 8. (a) Anthropomorphic medieval graves in Pena (Vila Real, north Portugal); (b) coastal defensive towers and Chapel of Santiago (9th and 12th centuries, respectively) in Catoria (Galicia, Spain); (c) example of the 13th century typology of porticoed urban building. Casa della Dogana (Padua, Italy).

\subsubsection{High Middle Ages}

The construction of the cathedral of Santiago de Compostela (Spain) began in 1098 [140]; new pilgrimage routes were opened, and the mendicant orders of Dominicans and Franciscans were born. For example, just in the city of Segovia (Spain), more than 20 Romanesque churches were built between 1180 and 1250 . Thus, specialized workforce was necessary. The master builders controlled the quality of the stones and they oversaw the construction and maintenance of the buildings. Some of the master builders were international experts and their recognition spread throughout Europe, transmitting their knowledge orally to the trade guilds. For example, the French master builder Giral Fruchel introduced the Gothic of the Île-de-France into the Iberian Peninsula, and he worked in the construction of several cathedrals in Spain [141].

Each artisan was member of a trade guild, which protected the artisans' interests and fixed the price of their products. The stonemason marks used to mark the work carried out by stonemasons are very frequent in churches and castles of this time (Figure 9). Members of the guild were classified as: "Apprentices" who were maintained trained by the Master for approximately 6 years; "Officers" who perfected their skills and charged for their work; and "Master builders" who would continue to improve their skills and could open their own workshop. The artisan guilds got the right to maintain a lodge, a place to meet and keep the plans of the building and other documents of the guilds. 

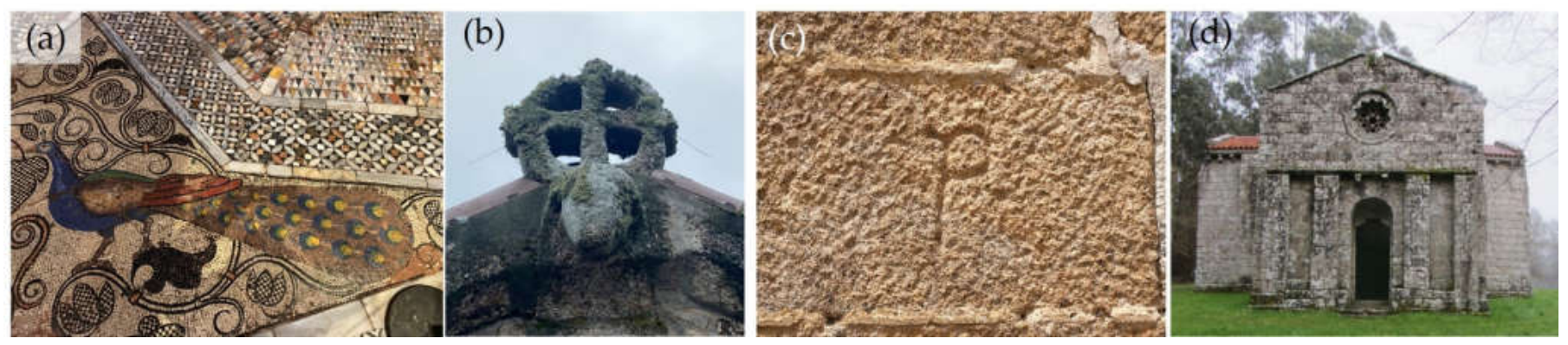

Figure 9. (a) Mosaic floor with peacocks in the Saint Mark's Basilica of Venice(12-13th century); (b) cross in the Romanesque apse of Sao Tiago church in Folhadela (Vila Real, northern Portugal) built with granite in the 12th century; (c) Stonemason marks in the Romanesque church of Nossa Senhora de Guadalupe of Mouçós (Vila Real, north Portugal); (d) San Miguel de Breamo Romanesque church (A Coruña, Spain) built with granite ashlars in the 12th century.

Romanesque architecture was characterized by new models of sculptural representation with great symbolic content (Figure 9b). At the beginning of the 12th century, two architectural innovations, the buttress and the pointed arch, made it possible to prop up walls and make them thinner, allowing them to support a greater weight. This transformation is visible in the Cistercian monasteries, which are in the transition between the Romanesque and Gothic styles. The construction of the first Gothic cathedrals began at the end of the 12th century. The complexity of Gothic buildings [142,143] has been a driving force behind the study of building stones, especially its mechanical resistance. Their columns and nerves had to withstand great pressure.

The Persian scholar Abu Rayhan al-Biruni (973-1048 AC) described the petrophysical properties of 100 known minerals, developing the hydrostatic weighing method to calculate the density of both ornamental as precious stones [144]. The most prominent lapidary is that of the Iranian aristocrat Muhammad Ibn Mansur. It was written in the 12th century and dedicated to Sultan Abbasi of Persia. Weight, hardness, variety and location of stones were described with precision in this treatise. Going back to Europe, the Toledo school of translators of Spain was founded at the beginning of the 12th century and important books written in Arabic were translated into Latin, which contributed to the dissemination of numerous books.

\subsubsection{Late Middle Ages}

The first translation of a lapidary was commissioned by Alfonso X "El Sabio" of Castille (1221-1284). Yehuda ben Moshe ha-Kohen translated it between 1243 and 1250. This lapidary deals with the magical properties of stones in relation to astrology. Albertus Magnus (1193/1206-1280) wrote De mineralibus (Minerals) consisting of five books on stones. These books describe the astrological influences and hidden powers of stones and the "chemistry" of the alchemists. It attempted to relate the limited and basic knowledge of the time about nature to the properties of stones. The Tafasi treatise (13th century) is divided into 24 chapters. It is based on the dictates of Aristotle and Belinas, on the genesis of each stone; and it describes their qualities, prices, varieties and defects. Books written in vernacular language (not in Latin) began to appear also in the 13th century. The best-known work is the travel notebook, dated between 1220 and 1240, of the French master builder Villard de Honnecourt (ca. 1200-ca. 1250), where there is a set of drawings, texts and teachings on building stones. This master builder worked on the construction of the Cistercian abbey of Vaucelles; on several cathedrals in France, such as Notre Dame de Reims, Meaux, Saint-Étienne, Chartres, and Lausanne; and on the cathedral of Košice (Hungary) [145]. 
A succession of rains and poor harvests led to famines in much of Europe between 1315 and 1318. Territorial struggles and revolts in all of Europe together with the Black Death that reached Europe in 1346 caused the death of approximately $60 \%$ of the population in the first half of the 14th century. Due to this crisis, a great deal of knowledge on building stone extraction and construction techniques was lost, with technical limitations in important buildings becoming apparent. Beauvais Cathedral collapsed at the end of the 13th century, and the Gothic cathedrals of Cologne (Germany), Narbonne (France), Siena (Italy) and Oviedo (Spain) were left unfinished due to the lack of funds.

Until the end of the Middle Ages, the use of building stones in the civil construction of cities was not generalized. This material was mostly reserved for religious and military buildings. The population built their houses with combustible materials for economic reasons; as a result, cities often suffered catastrophic fires. To combat this danger, the authorities of some countries enacted, from the end of the 14th century on, laws to impose stone construction.

\section{Modern History}

The economic collapse was a crucial cause for the development of the Renaissance in northern Italy [146]. The construction of the dome of Santa Maria del Fiore cathedral, in Florence (Italy), between 1420 and 1436 by Filippo Brunelleschi (1377-1446) is considered the beginning of the Renaissance. Brunelleschi drew up one of the first contracts in which a master builder specified the most important aspects of the construction and assumed responsibility for it in 1420 . The masonry dome over the Florentine cathedral is composed of eight ribs and eight webs: octagonal ribbed pointed dome. The lantern, which is a belvedere, is made of marble [147]. This stone has been widely used during the Italian Renaissance by sculptors and architects [148]. The Italian Leon Battista Alberti (1404-1472) designed the upper part of Santa Maria delle Carceri church in Prato (Italy) with inlaid green marble, also called "serpentino". He also designed the polychrome marble inlay façade of the church of Santa Maria Novella in Florence (Italy) and restored Santo Stefano Rotondo in Rome. Battista Alberti wrote De Re Ædificatoria (On the Art of Building) in 1452, a treatise on the theoretical and practical aspects of architecture, based on the work of Vitruvius. The second volume is dedicated to building materials.

\subsection{Early Modern Period}

Towns and cities had grown under the cover of old medieval castles built to withstand an attack with spears and non-powder propelled projectiles. However, the fall of Constantinople to the Turks (1453) and the invasion of Naples by French troops in 1492 marked the widespread use of gunpowder-propelled artillery. From then on, the villages and towns were vulnerable and needed to reinforce their walls [149]. These issues, together with the drop in temperatures $[150,151]$ led to the to the building of more solid walls and to a greater exploitation of quarries. Intensive construction activity and many publications on building materials, architecture and military urban planning were generated throughout Europe. The construction of forts required knowledge of quarries, of properties of building stones, on how the foundations, walls, pillars, columns and other stone elements should be, of mixtures of mortars, and of tips to save money [152]. Almost a score of treatises was published between 1554 and 1599 just in Italy (Table 1). The main European academies were created in the Renaissance (Table 2) due to the growing interest in the study of architecture. 
Table 1. Main treatises on fortification and architecture.

\begin{tabular}{|c|c|c|c|c|}
\hline Publication & Author & Author's Life & Title of the Book & Country \\
\hline ca. 1450 & $\begin{array}{l}\text { Leon Battista } \\
\text { Alberti }\end{array}$ & $1404-1472$ & De Re Ædificatoria & Italy \\
\hline 1465 & $\begin{array}{c}\text { Antonio di } \\
\text { Pietro Averlino }\end{array}$ & $1400-1469$ & Trattato di Architettura & Italy \\
\hline 1472 & Roberto Valturio & $1405-1475$ & De re militari libri XII & Italy \\
\hline 1493 & $\begin{array}{c}\text { Antonio } \\
\text { Cornazzano }\end{array}$ & $1429-1484$ & $\begin{array}{l}\text { Opera bellissima de l'arte } \\
\text { militare }\end{array}$ & Italy \\
\hline ca. 1478-1481 & \multirow{2}{*}{$\begin{array}{l}\text { Francesco di } \\
\text { Giorgio Martini }\end{array}$} & \multirow{2}{*}{ 1439-1501 } & $\begin{array}{c}\text { Trattato I (Architettura, } \\
\text { ingegneria e arte militare) }\end{array}$ & \multirow{2}{*}{ Italy } \\
\hline ca. 1490 & & & $\begin{array}{l}\text { Trattato II (Architettura } \\
\text { civile e militare) }\end{array}$ & \\
\hline$?$ & $\begin{array}{l}\text { Leonardo da } \\
\text { Vinci }\end{array}$ & $1452-1519$ & $\begin{array}{c}\text { Manuscripts (Codex } \\
\text { Madrid II) }\end{array}$ & Italy \\
\hline 1527 & Jacopo di Porcia & $1462-1538$ & De Re Militari & Italy \\
\hline 1527 & Albrecht Dürer & $1471-1528$ & $\begin{array}{l}\text { Etliche Underricht zu } \\
\text { Befestigung der Stett, } \\
\text { Schloss und Flecken }\end{array}$ & Germany \\
\hline 1529 & $\begin{array}{l}\text { Giovan Battista } \\
\text { della Valle }\end{array}$ & $1470-1550$ & $\begin{array}{c}\text { Vallo Libro continente } \\
\text { appertinentie à capitanii } \\
\ldots \text {. fortificare una citta ... }\end{array}$ & Italy \\
\hline 1536 & Daniel Specklin & ca. 1536-1589 & $\begin{array}{c}\text { Architectura von } \\
\text { Vestungen .... . } \\
\text { Bollwerken, Cavalieren, } \\
\text { Streichen ... }\end{array}$ & Germany \\
\hline 1536 & Diego de Salazar & $?$ & De Re Militari & Spain \\
\hline 1547 & Enrico Rivio & $?$ & $\begin{array}{l}\text { L'architettura delle } \\
\text { fabriche }\end{array}$ & Germany \\
\hline 1537-ca. 1575 & Sebastiano Serlio & $1475-1554$ & $\begin{array}{c}\text { I sette libri } \\
\text { dell'architettura-Della } \\
\text { castrametatione di Polibio } \\
\text { ridotta ... }\end{array}$ & Italy \\
\hline 1554 & \multirow{2}{*}{$\begin{array}{l}\text { Pietro di } \\
\text { Giacomo } \\
\text { Cataneo }\end{array}$} & \multirow{2}{*}{$\begin{array}{l}\text { ca. } 1510-c a . \\
1574\end{array}$} & $\begin{array}{l}\text { I quattro primi libri di } \\
\text { architettura }\end{array}$ & \multirow{2}{*}{ Italy } \\
\hline 1567 & & & $\begin{array}{l}\text { L'Architettura di Pietro } \\
\text { Cataneo Sanese }\end{array}$ & \\
\hline 1557 & $\begin{array}{l}\text { Giacomo Lanteri } \\
\text { di Paratico }\end{array}$ & & $\begin{array}{l}\text { Dil modo di fare le } \\
\text { fortificationi di terra ... } \\
\text { Città \& alle castella per } \\
\text { fortificarle }\end{array}$ & Italy \\
\hline 1558 & Jacopo Aconcio & 1492-ca.. 1566 & De Methodo & Italy \\
\hline 1559 & $\begin{array}{l}\text { Giacomo Lanteri } \\
\text { (Lantieri) }\end{array}$ & & $\begin{array}{l}\text { Due libri del modo di fare } \\
\text { le fortificationi di terra } \\
\text { intorno alle città \& alle } \\
\text { castella ... }\end{array}$ & Italy \\
\hline $1575-1591$ & $\begin{array}{l}\text { Alonso de } \\
\text { Vandelvira y } \\
\text { Luna }\end{array}$ & $1544-1626$ & $\begin{array}{l}\text { Tratado de arquitectura } \\
\text { sobre el arte de cortar la } \\
\text { piedra }\end{array}$ & Spain \\
\hline 1598 & $\begin{array}{l}\text { Cristóbal de } \\
\text { Rojas }\end{array}$ & 1555-1611 & $\begin{array}{l}\text { Teórica y práctica de } \\
\text { fortificación }\end{array}$ & Spain \\
\hline
\end{tabular}


Table 1. Cont.

\begin{tabular}{|c|c|c|c|c|}
\hline Publication & Author & Author's Life & Title of the Book & Country \\
\hline 1560 & $\begin{array}{c}\text { Giovanni } \\
\text { Battista Zanchi }\end{array}$ & $1515-1586$ & $\begin{array}{c}\text { Del modo di fortificar le } \\
\text { citta }\end{array}$ & Italy \\
\hline \multirow[b]{2}{*}{1564} & Girolamo Maggi & \multirow[b]{2}{*}{ ca. 1523-1572 } & \multirow[b]{2}{*}{$\begin{array}{l}\text { Della fortificatione delle } \\
\text { città }\end{array}$} & \multirow[b]{2}{*}{ Italy } \\
\hline & $\begin{array}{l}\text { Giacomo } \\
\text { Castriotto } \\
\text { (Japoco) }\end{array}$ & & & \\
\hline 1564 & \multirow{4}{*}{$\begin{array}{l}\text { Girolamo } \\
\text { Cataneo }\end{array}$} & \multirow{4}{*}{$1540-1584$} & $\begin{array}{l}\text { Opera nuova di fortificare, } \\
\text { offendere et difendere }\end{array}$ & \multirow{4}{*}{ Italy } \\
\hline 1571 & & & $\begin{array}{l}\text { Nuovo ragionamento del } \\
\text { fabricare le fortezze; si per } \\
\text { prattica }\end{array}$ & \\
\hline 1567 & & & Dell'Arte militare & \\
\hline 1600 & & & De Arte Bellica & \\
\hline 1567 & Domenico Mora & 1536-1586 & $\begin{array}{l}\text { In Dialogo sopra il Fare } \\
\text { batterie, foritificare una } \\
\text { citta. }\end{array}$ & Italy \\
\hline 1569 & & & $\begin{array}{l}\text { Discorsi delle } \\
\text { fortificazioni }\end{array}$ & \\
\hline 1584 & Carlo Theti & 1529-1589 & $\begin{array}{l}\text { Istruzione per i } \\
\text { Bombardieri }\end{array}$ & Italy \\
\hline 1585 & & & $\begin{array}{l}\text { Dell'Espugnazione e } \\
\text { difesa delle fortezze }\end{array}$ & \\
\hline 1570 & Galaaso Alghisi & $1523-1573$ & $\begin{array}{c}\text { Delle Fortificazioni ... } \\
\text { Libri Tre }\end{array}$ & Italy \\
\hline 1571 & $\begin{array}{l}\text { Girolamo } \\
\text { Cataneo }\end{array}$ & $1540-1584$ & $\begin{array}{l}\text { Nuovo rogionomento del } \\
\text { fabricare le fortezze }\end{array}$ & Italy \\
\hline 1572 & $\begin{array}{l}\text { Giacomo Fusto } \\
\text { Castriotto } \\
\text { (Jacopo) }\end{array}$ & $1510-1562$ & $\begin{array}{l}\text { Della fortificatione delle } \\
\text { città }\end{array}$ & Italy \\
\hline 1573 & $\begin{array}{l}\text { Jan van Schille } \\
\text { (Hans Schille) }\end{array}$ & $1510-1586$ & $\begin{array}{c}\text { Form und Weis zu } \\
\text { bauwen ... Vestung, } \\
\text { Schlosser, Burgen und } \\
\text { Stedt... }\end{array}$ & $\begin{array}{l}\text { The } \\
\text { Nether- } \\
\text { lands }\end{array}$ \\
\hline 1575 & $\begin{array}{l}\text { Vincenzo } \\
\text { Locatelli }\end{array}$ & ca. 1526-1584 & Invito Generale & Italy \\
\hline 1576 & $\begin{array}{l}\text { António } \\
\text { Rodrigues }\end{array}$ & $1525-1590$ & $\begin{array}{l}\text { Tratado de Arquitectura } \\
\text { (Manuscrito) }\end{array}$ & Portugal \\
\hline 1578 & Filippo Terzi & $1520-1597$ & $\begin{array}{c}\text { Estudos sobre } \\
\text { embadometria, } \\
\text { estereometria e ordens de } \\
\text { arquitectura }\end{array}$ & Portugal \\
\hline 1579 & $\begin{array}{l}\text { Marc Aurelio di } \\
\text { Passino }\end{array}$ & $?$ & $\begin{array}{c}\text { Discours sur ... de } \\
\text { l'architecture de guerre: } \\
\text { concernants ... l'artillerie }\end{array}$ & France \\
\hline 1580 & \multirow[t]{2}{*}{ Gabriello Busca } & \multirow[t]{2}{*}{ 1540-1605 } & $\begin{array}{l}\text { Della espugnatione et } \\
\text { difesa delle fortezze }\end{array}$ & \multirow[t]{2}{*}{ Italy } \\
\hline 1619 & & & L'Architettura Militare & \\
\hline
\end{tabular}


Table 1. Cont.

\begin{tabular}{|c|c|c|c|c|}
\hline Publication & Author & Author's Life & Title of the Book & Country \\
\hline 1582 & $\begin{array}{l}\text { Walter Herman } \\
\text { Ryff }\end{array}$ & $1500-1548$ & $\begin{array}{c}\text { Baukunst oder } \\
\text { Architektur aller } \\
\text { fürnemsten ... Künsten, } \\
\text { eygentlicher ... }\end{array}$ & Germany \\
\hline 1582 & \multirow{4}{*}{ Antonio Lupicini } & \multirow{4}{*}{ 1530-1592 } & $\begin{array}{l}\text { Architettura militare con } \\
\text { altri avvertimenti } \\
\text { appartenenti alla guerra }\end{array}$ & \multirow{4}{*}{ Italy } \\
\hline 1583 & & & $\begin{array}{c}\text { Discorsi militari } \\
\text { dell'eccellentissimo ... } \\
\text { Della Rovere duca } \\
\text { d'Urbino ... }\end{array}$ & \\
\hline 1583 & & & Diálogos del arte militar & \\
\hline 1587 & & & $\begin{array}{l}\text { Discorsi di architettura } \\
\text { militare }\end{array}$ & \\
\hline 1583 & $\begin{array}{l}\text { Diego García de } \\
\text { Palacio }\end{array}$ & $1540-1595$ & Diálogos militares & Spain \\
\hline 1585 & $\begin{array}{l}\text { Giacomo } \\
\text { Aconcio }\end{array}$ & $1492-1584$ & $\begin{array}{l}\text { Ars muniendorum } \\
\text { oppidorum }\end{array}$ & Italy \\
\hline 1587 & \multirow[b]{2}{*}{ Ambroise Bachot } & \multirow[b]{2}{*}{$\begin{array}{l}\text { ca. } 1540-c a . \\
1600\end{array}$} & Le Timon & \multirow[b]{2}{*}{ France } \\
\hline 1598 & & & $\begin{array}{l}\text { Le gouvernail ... } \\
\text { l'architecture des } \\
\text { fortifications ... }\end{array}$ & \\
\hline 1588 & $\begin{array}{l}\text { Agostino } \\
\text { Ramelli }\end{array}$ & $1531-1608$ & $\begin{array}{l}\text { Le diverse et artificiose } \\
\text { machine }\end{array}$ & Italy \\
\hline 1589 & Paul Ive & $?-1604$ & $\begin{array}{l}\text { The practise of } \\
\text { fortification }\end{array}$ & England \\
\hline 1594 & $\begin{array}{l}\text { Giulio di } \\
\text { Savorgnan }\end{array}$ & 1510-1595 & $\begin{array}{l}\text { Venticinque regole per la } \\
\text { fortificazione }\end{array}$ & Italy \\
\hline 1594 & \multirow{4}{*}{ Simon Stevin } & \multirow{4}{*}{$1548-1620$} & De Sterktenbouwing & \multirow{4}{*}{$\begin{array}{l}\text { The } \\
\text { Nether- } \\
\text { lands }\end{array}$} \\
\hline 1649 & & & $\begin{array}{l}\text { Van de oirdeningh der } \\
\text { steden }\end{array}$ & \\
\hline 1617 & & & $\begin{array}{l}\text { Nieuwe Maniere van } \\
\text { Stercktebou door } \\
\text { Spilsluysen }\end{array}$ & \\
\hline 1649 & & & $\begin{array}{c}\text { Materiæ Politicæ, } \\
\text { Burgherlicke Stoffen }\end{array}$ & \\
\hline 1596 & Giovanni Scala & 1547-1599 & Delle fortificationi & Italy \\
\hline 1597 & Bonaiuto Lorini & $1540-1611$ & $\begin{array}{l}\text { Delle fortificationi libri } \\
\text { cinque }\end{array}$ & Italy \\
\hline 1598 & $\begin{array}{l}\text { Giovanni } \\
\text { Battista Bellucci }\end{array}$ & $1506-1554$ & $\begin{array}{l}\text { Nuova inventione di } \\
\text { fabricar fortezze di varie } \\
\text { forme }\end{array}$ & Italy \\
\hline 1599 & $\begin{array}{l}\text { Francesco de } \\
\text { Marchi }\end{array}$ & $1504-1577$ & $\begin{array}{c}\text { Trattato di Architettura } \\
\text { Militare }\end{array}$ & Italy \\
\hline 1599 & $\begin{array}{l}\text { Diego González } \\
\text { de Medina Barba }\end{array}$ & ca.. 1550-1600 & Examen de fortificación & Spain \\
\hline 1599 & Charles de Beste & $?$ & De Architectura & $\begin{array}{l}\text { The } \\
\text { Nether- } \\
\text { lands }\end{array}$ \\
\hline
\end{tabular}


Table 1. Cont.

\begin{tabular}{|c|c|c|c|c|}
\hline Publication & Author & Author's Life & Title of the Book & Country \\
\hline 1599 & $\begin{array}{l}\text { Giovanni } \\
\text { Pomodoro }\end{array}$ & $?$ & $\begin{array}{l}\text { Geometria prattica tratta } \\
\text { dagl' Elementi d' Euclide } \\
\text { et altri autori }\end{array}$ & Italy \\
\hline 1600 & $\begin{array}{l}\text { Jean Errard de } \\
\text { Bar-le-Duc }\end{array}$ & $1554-1626$ & $\begin{array}{c}\text { La fortification } \\
\text { demonstree et reduicte en } \\
\text { art }\end{array}$ & France \\
\hline 1601 & Jacques Perret & $1540-1619$ & $\begin{array}{c}\text { Des Fortifications et } \\
\text { Artifices Architecture et } \\
\text { Perspective }\end{array}$ & France \\
\hline 1602 & Mateo Morán & $?$ & Nuevo modo de fortificar & Spain \\
\hline 1604 & \multirow{2}{*}{$\begin{array}{l}\text { Giovanni } \\
\text { Francesco } \\
\text { Fiammelli }\end{array}$} & \multirow[t]{2}{*}{$1565-1613$} & $\begin{array}{l}\text { Il Principe difeso, nel } \\
\text { quale si tratta di } \\
\text { Fortificazione ... }\end{array}$ & Italy \\
\hline 1606 & & & I quesiti militari & \\
\hline 1607 & \multirow{4}{*}{$\begin{array}{c}\text { Johann Wilhelm } \\
\text { Dilich }\end{array}$} & \multirow{4}{*}{$1571-1650$} & $\begin{array}{l}\text { Kriegsbuch: darin die alte } \\
\text { und neue Militia } \\
\text { eigentlich beschrieben un } \\
\text { alle ... }\end{array}$ & \multirow{4}{*}{ Germany } \\
\hline 1640 & & & $\begin{array}{c}\text { Peribologia } \\
\text { (Vestungsgebewen) }\end{array}$ & \\
\hline 1641 & & & $\begin{array}{c}\text { Peribologia seu } \\
\text { Muniendorum locorum } \\
\text { ratio }\end{array}$ & \\
\hline 1645 & & & $\begin{array}{l}\text { Kurtzer und in Tabulis } \\
\text { verfassete underricht ... } \\
\text { Bollwercke ... Anzulegen }\end{array}$ & \\
\hline 1611 & $\begin{array}{l}\text { Cristóbal } \\
\text { Lechuga }\end{array}$ & $1557-1622$ & $\begin{array}{l}\text { Discurso que trata de la } \\
\text { artillería ... con un } \\
\text { tratado de fortificación y } \\
\text { otros ... }\end{array}$ & Spain \\
\hline 1611 & Claude Flamand & $1570-1613$ & $\begin{array}{l}\text { La guide des fortifications } \\
\text { et conduitte millitaire }\end{array}$ & Italy \\
\hline 1627 & \multirow[t]{2}{*}{ Samuel Marolois } & \multirow[t]{2}{*}{$1572-1627$} & $\begin{array}{c}\text { Fortification ou } \\
\text { Architecture militaire tant } \\
\text { offensive que defensive }\end{array}$ & \multirow{2}{*}{$\begin{array}{l}\text { The } \\
\text { Nether- } \\
\text { lands }\end{array}$} \\
\hline 1633 & & & $\begin{array}{l}\text { Fortificationis sive artis } \\
\text { muniendi }\end{array}$ & \\
\hline 1614 & $\begin{array}{c}\text { Mario } \\
\text { Savorgnan }\end{array}$ & $1513-1574$ & $\begin{array}{c}\text { Arte Militare Terrestre, e } \\
\text { Maritima; Secondo la } \\
\text { Ragione ... Itália }\end{array}$ & Italy \\
\hline 1615 & $\begin{array}{l}\text { Vincenzo } \\
\text { Scamozzi }\end{array}$ & $1548-1618$ & $\begin{array}{c}\text { L'Idea della Architettura } \\
\text { Universale }\end{array}$ & Italy \\
\hline 1618 & $\begin{array}{l}\text { Alexander von } \\
\text { Grotte }\end{array}$ & $1599-1637$ & $\begin{array}{c}\text { Neue Manier mit wenigen } \\
\text { Kosten Festungen } \mathrm{zu} \\
\text { bauen }\end{array}$ & Germany \\
\hline
\end{tabular}


Table 1. Cont.

\begin{tabular}{|c|c|c|c|c|}
\hline Publication & Author & Author's Life & Title of the Book & Country \\
\hline 1618 & \multirow{4}{*}{ Pietro Sardi } & \multirow{4}{*}{$1560-1642$} & $\begin{array}{c}\text { Corona imperiale } \\
\text { dell'architettura militare }\end{array}$ & \multirow{4}{*}{ Italy } \\
\hline 1627 & & & $\begin{array}{l}\text { Discorso, per il quale con } \\
\text { vive e certe ragioni si } \\
\text { rifiutano tutte le fortezze }\end{array}$ & \\
\hline 1639 & & & $\begin{array}{l}\text { Corno dogale della } \\
\text { architettura militare }\end{array}$ & \\
\hline 1642 & & & $\begin{array}{c}\text { Discorso sopra la } \\
\text { necessità \& utilità } \\
\text { dell'architettura militare }\end{array}$ & \\
\hline 1620 & $\begin{array}{l}\text { Pietro Antonio } \\
\text { Barca }\end{array}$ & $1586-1636$ & $\begin{array}{c}\text { Avvertimenti' e regole } \\
\text { circa l'architettura civile, } \\
\text { scultura ... et architettura } \\
\ldots\end{array}$ & Italy \\
\hline$?$ & $\begin{array}{c}\text { Giovanni de } \\
\text { Galliano Pieroni }\end{array}$ & $1586-1654$ & $\begin{array}{c}\text { Trattato delle } \\
\text { fortificazioni moderne }\end{array}$ & Italy \\
\hline 1622 & $\begin{array}{l}\text { Leonardo } \\
\text { Torriani } \\
\text { (Turriano) }\end{array}$ & $1559-1628$ & $\begin{array}{c}\text { Dos discursos de } \\
\text { Leonardo Turriano .... }\end{array}$ & Portugal \\
\hline 1624 & Robert Fludd & $1574-1637$ & De arte militari & England \\
\hline 1624 & $\begin{array}{l}\text { Francesco } \\
\text { Tensini }\end{array}$ & $1581-1638$ & $\begin{array}{c}\text { La fortificatione, guardia, } \\
\text { difesa, et espugnatione } \\
\text { delle fortezze... }\end{array}$ & Italy \\
\hline 1626 & $\begin{array}{c}\text { Adriaan } \\
\text { Adriaansz } \\
\text { (Adriaan } \\
\text { Metius/Anthonis }\end{array}$ & on) & $\begin{array}{l}\text { Maet-constigh liniael ofte } \\
\text { proportionalen ry ende } \\
\text { platten passer }\end{array}$ & $\begin{array}{l}\text { The } \\
\text { Nether- } \\
\text { lands }\end{array}$ \\
\hline 1627 & Matteo Oddi & $1576-1626$ & $\begin{array}{l}\text { Precetti di architettura } \\
\text { militare }\end{array}$ & Italy \\
\hline 1630 & $\begin{array}{l}\text { Pietro Paolo } \\
\text { Floriani }\end{array}$ & $1585-1638$ & $\begin{array}{l}\text { Diffesa et offesa delle } \\
\text { piazze }\end{array}$ & Italy \\
\hline 1630 & \multirow{2}{*}{$\begin{array}{c}\text { Joseph } \\
\text { Furttenbach }\end{array}$} & \multirow{2}{*}{$1591-1667$} & Architectura martialis & \multirow{2}{*}{ Germany } \\
\hline 1635 & & & Architectura universalis & \\
\hline 1631 & $\begin{array}{c}\text { Adam Fritach } \\
\text { (Freitag, Freytag) }\end{array}$ & $1608-1650$ & $\begin{array}{c}\text { Architectura militaris } \\
\text { nova et aucta, oder Newe } \\
\text { vermehrte Fortification } \\
\ldots\end{array}$ & \multirow[t]{2}{*}{$\begin{array}{l}\text { The } \\
\text { Nether- } \\
\text { lands }\end{array}$} \\
\hline 1639 & & & $\begin{array}{c}\text { L'Architecture militaire } \\
\text { ou la fortification } \\
\text { nouvelle }\end{array}$ & \\
\hline 1631 & $\begin{array}{l}\text { Matheus do } \\
\text { Couto, O velho }\end{array}$ & $1616-1676$ & Tractado de Architectura & Portugal \\
\hline 1633 & \multirow{2}{*}{$\begin{array}{l}\text { Johann } \\
\text { Faulhaber } \\
\text { (Johannes) }\end{array}$} & \multirow{2}{*}{$1580-1638$} & Academia fortificatoria & \multirow[t]{2}{*}{ Germany } \\
\hline 1637 & & & Ingenieurschul & \\
\hline 1636 & Galileo Galilei & $1564-1642$ & Trattato di fortificazione & \multirow{2}{*}{ Italy } \\
\hline 1639 & Giuseppe Barca & $1595-1639$ & $\begin{array}{l}\text { Breue compendio di } \\
\text { fortificatione moderna }\end{array}$ & \\
\hline
\end{tabular}


Table 1. Cont.

\begin{tabular}{|c|c|c|c|c|}
\hline Publication & Author & Author's Life & Title of the Book & Country \\
\hline 1640 & Antoine de Ville & 1596-1656 & $\begin{array}{l}\text { Les Fortifications du } \\
\text { Chevalier Antoine de } \\
\text { Ville }\end{array}$ & France \\
\hline 1643 & \multirow{3}{*}{$\begin{array}{l}\text { Nicolaus } \\
\text { Goldmann } \\
\text { (Nikolaus, } \\
\text { Nicolas) }\end{array}$} & \multirow{3}{*}{$1611-1665$} & $\begin{array}{c}\text { Elementorum } \\
\text { architecturae militaris } \\
\text { libri IV }\end{array}$ & \multirow{3}{*}{$\begin{array}{l}\text { The } \\
\text { Nether- } \\
\text { lands }\end{array}$} \\
\hline 1643 & & & $\begin{array}{l}\text { La nouvelle fortification } \\
\text { Polônia Holanda }\end{array}$ & \\
\hline 1656 & & & $\begin{array}{l}\text { Tractatus de usu } \\
\text { proportionatorii sive } \\
\text { circini proportionalis }\end{array}$ & \\
\hline 1644 & $\begin{array}{c}\text { Don Juan de } \\
\text { Santans y Tapia }\end{array}$ & $?-1658$ & $\begin{array}{l}\text { Tratado de fortificación } \\
\text { militar de estos tiempos }\end{array}$ & Spain \\
\hline 1645 & $\begin{array}{l}\text { Blaise François } \\
\text { Pagan }\end{array}$ & 1604-1665 & Les Fortifications & France \\
\hline 1647 & Matthias Dögen & 1605-1676 & $\begin{array}{c}\text { Architectura militaris } \\
\text { moderna: variis historiis, } \\
\text { tam veteribus ... }\end{array}$ & $\begin{array}{l}\text { The } \\
\text { Nether- } \\
\text { lands }\end{array}$ \\
\hline 1650 & Cosimo Noferi & $1635-1661$ & $\begin{array}{l}\text { La Travagliata } \\
\text { Architettura }\end{array}$ & Italy \\
\hline 1651 & $\begin{array}{l}\text { Diego Enríquez } \\
\text { de Villegas }\end{array}$ & $1600-1671$ & $\begin{array}{c}\text { Academia de fortificación } \\
\text { de plazas }\end{array}$ & Spain \\
\hline 1654 & Henrik Ruse & $1624-1679$ & $\begin{array}{c}\text { Versterckte vesting, } \\
\text { uitgevonden in velerleij } \\
\text { voorvallen ... }\end{array}$ & $\begin{array}{l}\text { The } \\
\text { Nether- } \\
\text { lands }\end{array}$ \\
\hline 1654 & George Fournier & 1595-1652 & $\begin{array}{c}\text { Traité des fortifications, } \\
\text { ou Architecture militaire } \\
\ldots\end{array}$ & France \\
\hline 1655 & Pierre Bourdin & $1595-1653$ & $\begin{array}{l}\text { L'architecture militaire, } \\
\text { ou l'art de fortifier les } \\
\text { places régulières ... }\end{array}$ & France \\
\hline 1657 & \multirow{3}{*}{$\begin{array}{l}\text { Gennaro Maria } \\
\text { d'Afflitto }\end{array}$} & \multirow{3}{*}{$1618-1673$} & $\begin{array}{l}\text { Compendio de Modernas } \\
\text { Fortificaciones }\end{array}$ & \multirow{3}{*}{ Italy } \\
\hline 1665 & & & $\begin{array}{l}\text { Breve trattato delle } \\
\text { moderne fortificazioni }\end{array}$ & \\
\hline 1667 & & & $\begin{array}{l}\text { Introduzione alla } \\
\text { moderna fortificazione } \\
\text { cavata }\end{array}$ & \\
\hline 1659 & $\begin{array}{l}\text { Christoph } \\
\text { Notnagel }\end{array}$ & 1607-1666 & $\begin{array}{c}\text { Manuale fortificstorium, } \\
\text { oder, Kurtzes } \\
\text { handbuchlein ... }\end{array}$ & Germany \\
\hline 1659 & \multirow{2}{*}{$\begin{array}{l}\text { Luís Serrão } \\
\text { Pimentel }\end{array}$} & $1613-1679$ & $\begin{array}{c}\text { Architectonica Militar ou } \\
\text { Fortificação Moderna }\end{array}$ & \multirow{2}{*}{ Portugal } \\
\hline 1680 & & & $\begin{array}{c}\text { Methodo Lusitanico de } \\
\text { Desenhar as fortificaçoens } \\
\text { das Praças ... }\end{array}$ & \\
\hline 1661 & Juan de Torija & $1624-1672$ & $\begin{array}{l}\text { Breve tratado de todo } \\
\text { genero de bohedas ... }\end{array}$ & Spain \\
\hline 1664 & $\begin{array}{l}\text { Christoph } \\
\text { Heydmann }\end{array}$ & $1635-1707$ & $\begin{array}{c}\text { Fortifikations } \\
\text { manier-Addimentum } \\
\text { Architecturæ militaris }\end{array}$ & Germany \\
\hline
\end{tabular}


Table 1. Cont.

\begin{tabular}{|c|c|c|c|c|}
\hline Publication & Author & Author's Life & Title of the Book & Country \\
\hline 1666 & Jean du Breuil & $1601-1670$ & $\begin{array}{c}\text { L'art ... des fortifications } \\
\text { françaises, hollandoises, } \\
\text { espagnoles, italiennes ... }\end{array}$ & France \\
\hline 1666 & $\begin{array}{l}\text { Johann } \\
\text { Matthäus } \\
\text { Faulhaber }\end{array}$ & $1604-1683$ & $\begin{array}{c}\text { Fortifikation-Manual zu } \\
\text { den Grundregeln der } \\
\text { Ingenieurskunst }\end{array}$ & Germany \\
\hline 1672 & \multirow{2}{*}{$\begin{array}{l}\text { Johann Bernhard } \\
\text { Scheither }\end{array}$} & \multirow[b]{2}{*}{$?-1677$} & Novissima Praxis militaris & \multirow{2}{*}{ Germany } \\
\hline 1688 & & & $\begin{array}{c}\text { Manuale architecturae } \\
\text { militaris }\end{array}$ & \\
\hline$?$ & $\begin{array}{c}\text { Manuel } \\
\text { Fernández de } \\
\text { Villarreal }\end{array}$ & & $\begin{array}{l}\text { Architetura militar o } \\
\text { Fortificacion moderna }\end{array}$ & Spain \\
\hline 1660 & $\begin{array}{l}\text { João Nunes } \\
\text { Tinoco }\end{array}$ & $1610-1689$ & $\begin{array}{c}\text { Taboadas gerais para } . . . \\
\text { obra do officio de } \\
\text { pedreiro }\end{array}$ & Portugal \\
\hline 1664 & $\begin{array}{l}\text { Vicente Mut } \\
\text { Armengol }\end{array}$ & $1614-1685$ & Arquitectura militar & Spain \\
\hline 1669 & $\begin{array}{l}\text { Alonso de } \\
\text { Zepeda y } \\
\text { Adrada }\end{array}$ & $?$ & $\begin{array}{c}\text { Epitome de la fortificación } \\
\text { moderna ... }\end{array}$ & Spain \\
\hline 1671 & $\begin{array}{l}\text { Pedro Antonio } \\
\text { Ramón Folch de } \\
\text { Cardona }\end{array}$ & $1611-1690$ & Geometria Militar & Spain \\
\hline 1673 & \multirow{3}{*}{ Georg Rimpler } & \multirow{3}{*}{$1636-1683$} & $\begin{array}{l}\text { Neü-herfürgegebene } \\
\text { Kriegsarchitectur }\end{array}$ & \multirow{3}{*}{ Germany } \\
\hline 1673 & & & $\begin{array}{l}\text { Ein dreyfacher Tractat } \\
\text { von den Festungen }\end{array}$ & \\
\hline 1674 & & & $\begin{array}{l}\text { Befestigte Festung, } \\
\text { Artillerie und Infanterie } \\
\text { mit drei Treffen ... }\end{array}$ & \\
\hline 1676 & $\begin{array}{l}\text { Annibale } \\
\text { Marchese } \\
\text { Porroni }\end{array}$ & $1623-1684$ & $\begin{array}{l}\text { Trattato universale } \\
\text { militare moderno }\end{array}$ & Italy \\
\hline 1675 & $\begin{array}{l}\text { Francesco } \\
\text { Eschinardi } \\
\text { (Costanzo } \\
\text { Amichevoli) }\end{array}$ & $1623-1703$ & $\begin{array}{c}\text { Architettura militare } \\
\text { ridotta a metodo facile e } \\
\text { breve }\end{array}$ & Italy \\
\hline 1677 & $\begin{array}{l}\text { Johann-Frantz } \\
\text { Griendel Von } \\
\text { Ach }\end{array}$ & $1631-1687$ & $\begin{array}{l}\text { Nova architectura } \\
\text { militaris }\end{array}$ & Germany \\
\hline 1678 & Donato Rossetti & 1633-1706 & Fortificazioni a rovescio & Italy \\
\hline 1682 & \multirow{2}{*}{$\begin{array}{l}\text { Menno van } \\
\text { Coehoorn }\end{array}$} & \multirow[t]{2}{*}{ 1641-1704 } & $\begin{array}{c}\text { Versterchinge de } \\
\text { Vijfhoeks met alle syne } \\
\text { Buytenwerken }\end{array}$ & \multirow{2}{*}{$\begin{array}{l}\text { The } \\
\text { Nether- } \\
\text { lands }\end{array}$} \\
\hline 1685 & & & $\begin{array}{l}\text { Nieuwe Vestingbouw op } \\
\text { een natte of lage horisont }\end{array}$ & \\
\hline 1683 & $\begin{array}{l}\text { Jean-François } \\
\text { Bernard }\end{array}$ & & $\begin{array}{l}\text { Nouvelle manière de } \\
\text { fortifier les places }\end{array}$ & France \\
\hline
\end{tabular}


Table 1. Cont.

\begin{tabular}{|c|c|c|c|c|}
\hline Publication & Author & Author's Life & Title of the Book & Country \\
\hline 1685 & \multirow{2}{*}{$\begin{array}{l}\text { Sébastien Le } \\
\text { Prestre de } \\
\text { Vauban }\end{array}$} & \multirow{2}{*}{$1635-1707$} & $\begin{array}{l}\text { Le Directeur général des } \\
\text { fortifications }\end{array}$ & \multirow{2}{*}{ France } \\
\hline 1704 & & & $\begin{array}{l}\text { Traité des sièges et de } \\
\text { l'attaque des places }\end{array}$ & \\
\hline ca.. 1690 & $\begin{array}{l}\text { Manuel Pinto de } \\
\text { Vilalobos }\end{array}$ & $?-1734$ & $\begin{array}{l}\text { Tractado do uzo do } \\
\text { pantometra de desenhar } \\
\text { as forteficasoins }\end{array}$ & Portugal \\
\hline 1693 & Jose Chafrion & $1653-1698$ & $\begin{array}{l}\text { Escuela de Palas ò sea } \\
\text { curso mathematico }\end{array}$ & Spain \\
\hline 1694 & $\begin{array}{l}\text { Giusto Emilio } \\
\text { Alberghetti }\end{array}$ & $1666-1755$ & $\begin{array}{l}\text { Compendio della } \\
\text { fortificatione }\end{array}$ & Italy \\
\hline 1696 & $\begin{array}{c}\text { Alain Manesson } \\
\text { Mallet }\end{array}$ & 1630-1706 & $\begin{array}{l}\text { Les Travaux de Mars ou } \\
\text { l'Art de la Guerre }\end{array}$ & France \\
\hline ca.. 1705 & Pêro Araújo & ?-1715 & $\begin{array}{l}\text { Tratado de Arquitectura } \\
\text { Política e Militar }\end{array}$ & Portugal \\
\hline
\end{tabular}

Table 2. First main European academies.

\begin{tabular}{ccc}
\hline Year & City-Country & Name \\
\hline 1583 & Madrid-Spain & Royal Mathematica Academy \\
1593 & Rome-Italy & Academy of San Luca \\
1603 & Rome-Italy & Linceana Academy \\
1648 & Paris-France & Royal Academy of Painting and Sculpture \\
ca. 1657 & Florence-Italy & Experiment Academy \\
1662 & Lonson-England & Royal Society of London for Improving \\
1666 & Paris-France & Natural Knowledge \\
1666 & Rome-Italy & Sciences Academy \\
1727 & Cortona-Italy & French Academy in Rome \\
1738 & Madrid-Spain & Etrusian Academy \\
1740 & Vatinan & Royal Academy of History \\
1752 & Madrid-Spain & Royal Academy of Fine Arts of San Fernando \\
1755 & Naples-Italy & Royal Herculanense Academy \\
1768 & Lonson-England & Royal Academy of Arts \\
1779 & Lisbon-Portugal & Lisbon Academy of Sciences \\
1808 & Munich-Germany & Royal Academy of Fine Arts \\
1808 & Amsterdam-The & Royal Netherlands Academy of Arts \\
1836 & Netherlands & and Sciences \\
& Lisbom-Portugal & National Academy of Fine Arts \\
\hline
\end{tabular}

The dimension stones were not exclusively destined to religious buildings (Figure 10) in this time. The powerful families built great palaces throughout Europe where the building stones acquired a marked decorative character in addition to their structural function. Spanish architect Diego de Sagredo (1490-1528) wrote Las Medidas del Romano (The Roman Measurements) in 1526, the first book on architecture and building materials printed in Spanish and the first original text published on the subject outside of Italy during the Renaissance. This book was translated into French and published in many countries. 

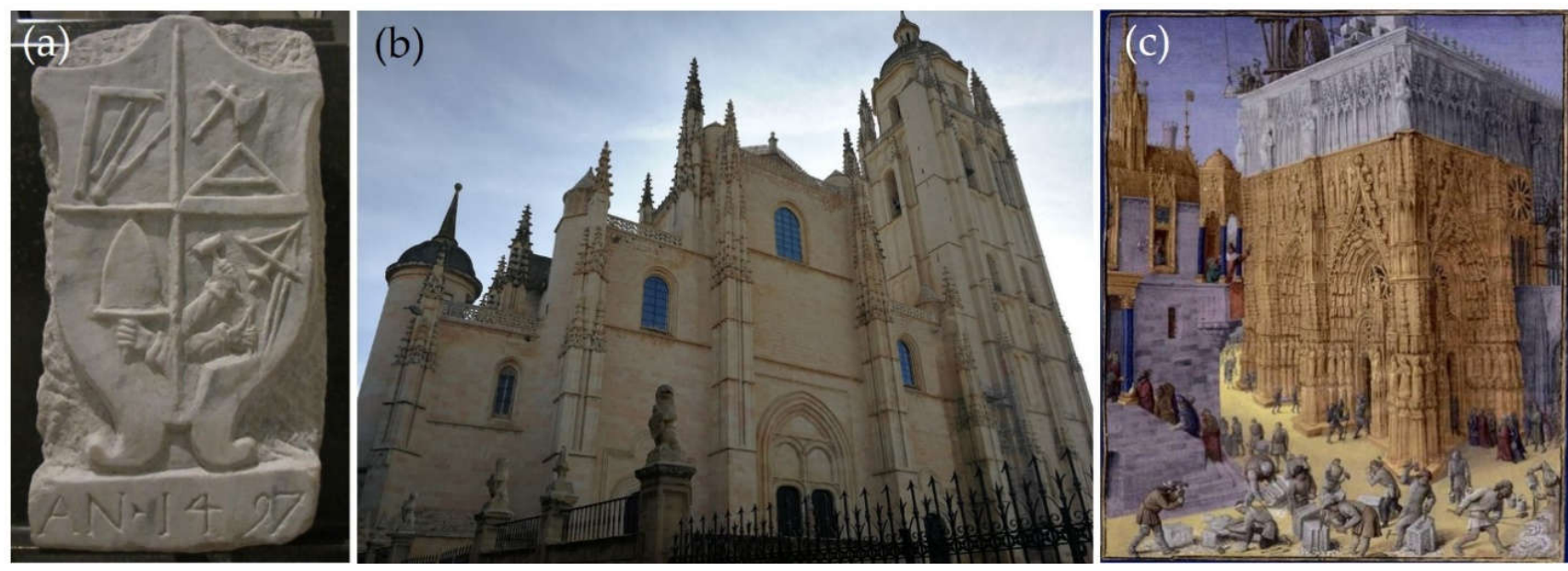

Figure 10. (a) Coat of arms of the brotherhood of bricklayers (1497) of the church of Santa María Magdalena, preserved in the Cathedral of Capua, Chapel of the Body of Christ, Diocesan museum (Italy); (b) Cathedral of Segovia (Spain) built in a Gothic style in the mid-16th century; (c) miniature representing the construction of the temple of Jerusalem-Fouquet Jean (1470-75) National Library of Paris (Richelieu).

Andrés de Vandelvira (ca. 1505-1575) was a Spanish architect and stonemason, he read the most famous treatises of his time and has a forerunner of the handkerchief vault and other architectural solutions. His son, Alonso de Vandelvira y Luna (1544-1626), wrote Tratado de arquitectura sobre el arte de cortar la Piedra (Architectural Treatise on the Art of Cutting Stone) between 1575 and 1591. It synthesized the stonework and constructive knowledge of his family.

The construction of San Lorenzo de El Escorial Royal Monastery (Madrid, Spain) between 1563 and 1584 constituted a milestone in the exploration and evaluation of building stones in Spain [134]. For its ornamentation, Spanish marbles were used and to cut them, a stonecutting workshop was built below the Monastery orchard [153,154]. Felipe II hired Flemish and French experts to locate the slate quarries used on the roof of the monastery. In the same period, "Opificio delle Pietre Dure" was an established in Florence (Italy) in 1588. It was a workshop dedicated to the development of the Byzantine technique called "commesso"; similar to mosaics, it consists of the inlaying of thin stone plates selected for their colour, opacity, gloss and grain to create elaborate decorative effects like those of the Cappella dei Principi (Chapel of the prince) (1602) in San Lorenzo basilica of Florence.

\subsubsection{First "Scientific Investigations"}

The French writer and poet François de Belleforest (1530-1583) wrote Cosmography, printed in 1575. It describes the figures of Rouffignac Cave (Dordogne, France), being the first reference to rock cave painting. A few years later, the Swiss jurist and art collector Basilius Amerbach (1533-1591) and Andreas Ryff (1550-1603) participated in the archaeological excavations of the Augusta Raurica theater (Switzerland) between 1588 and 1591. The Italian Michele Mercati (1541-1593) wrote Metallotheca Vaticana, published in 1719. This book described the stones and fossils of the Vatican museum, indicating that the "lightning stones" or "cerauni", considered to be of celestial origin until that time, could have been manufactured by humans. The Bolognese Ferdinando Cospi (1606-1686) published a full description of the "Cospiano Museum", a cabinet of curiosities, in five volumes in 1667. The first two described the natural history specimens, and the last three covered the archaeological objects. Although lacking a fully technical and methodological approach, these firsts studies show a systematic way of proceeding, and therefore, can already be considered "scientific" in analyzing the archaeological sites and heritage stones.

The French Bernard de Jussieu (1699-1777) published Origin and Uses of the Lightning Stone in 1723 to defend the human origin of arrowheads, axes and other tools. Seven years later, the also French Nicolas Mahudel (1673-1747), a physician, antiquarian and 
numismatist, tried to demonstrate the same to the Academy of Inscriptions and Fine Letters of France, referring to Michele Mercati and comparing tools from one side of the Atlantic Ocean to the other. Nicolas Mahudel also suggested, in 1734, that prehistoric tools changed from being made of stone to being made of bronze and iron.

\subsubsection{The "Archaeologist" King}

King Carlos VII of Naples (1734-1759), future King Carlos III of Spain (1759-1788) sponsored and closely followed the studies of the Spanish engineer Roque Joaquín de Alcubierre (1702-1780), who began the excavation and exploration of Herculaneum (Italy) in 1738 and of Pompeii (Italy) in 1748. The king created a museum in the Royal Villa of Portici in 1751 and the Regale Accademia Ercolanense in 1755, both in Naples, where specialists in restoration of marbles, bronzes and papyri studied the discovered pieces. The eight volumes of Le antichità di Ercolano Esposte (Herculaneum's Antiquity Exposed) began to be published in 1753, reproducing engravings of the recovered pieces, as well as plans and designs of the buildings that were coming to light [155]. King Carlos III also sponsored studies on "verracos", zoomorphic sculptures carved in granite around the 5th century BC, phoenician antiquities in Malaga and the Tránsito Synagogue in Toledo (Spain). He commissioned the architect Juan de Villanueva to build the Royal Cabinet of Natural History in Madrid (today Prado museum), founded in 1771 and which would house an important collection of lapidaries from all over the Spanish geography. Carlos III also and initiated the first archaeological program carried out in Mexico, the excavation of the city of Palenque [156].

King Carlos III hired Italian experts to continue with the exploration of ornamental stone quarries for the Royal Palace of Madrid (Spain) in 1761. Their reports were scientifically rigorous and included information about stones, such as their color, rarity, prevailing quality, and abundance, as well as the distance of their quarries to Madrid [157]. Carlos III approved the Instruction for the New Paving and Cleaning of the Streets of Madrid, which Substantially Contains the Don Francisco Sabatini Project. The Italian architect Francisco Sabatini planned to experimentally pave a central street in Madrid. One half would be paved with flint, a material with which the streets of Madrid had been paved since Arab times, and the other half with granite slabs, in the manner of the basalt slabs used in Naples (Italy). The durability and maintenance cost of both construction materials would be compared with this instruction. This represented the first modern tests of durability and quality of construction materials in Spain.

\subsubsection{The "Grand Tour"}

The term "Grand Tour" appeared written for the first time in 1670, in Voyage d'Italie (Trip to Italy) by Richard Lassels (ca. 1603-1668). It refers to the scholarly and leisure trip that young aristocrats, mainly British, made through Europe. The scientific advances and growing interest in heritage stones, contributed to including France, Spain and Italy in it. This trip became fashionable among the upper classes of Europe in the second half of the 18th century. The Grand Tour would foster interest in the research on ancient buildings and their stones. The publications of William Bromley, 1702 [158]; Juan Álvarez de Colmenar, 1707 [159]; Francis Carter, 1777 [160]; Richard Twiss, 1775 [161]; Henry Swinburne, 1779 [162]; Maurice Margarot, 1780 [163]; John Talbot Dillon, 1782 [164]; Joseph Townsend, 1791 [165]; Jean-Marie-Jérôme Fleuriot de Langle, 1785 [166]; and George Graydon [167] are some examples of the description of trips made in Spain. Explorers such as the Germans Alexander von Humboldt (1769-1859) and Maximilian Zu Wied-Neuwied (1782-1867) explored America [168]. The reading of historical documents reveals important data on heritage stones and the location of historical quarries. For example, the book Viaje de España (Travel through Spain) by Antonio Ponz, 1781 [169] facilitated the rediscovery of the historical quarries of the four fountains of the Paseo del Prado of Madrid (Spain) in 2017 [170]. 
King Gustav III of Sweden (1746-1792) made a trip in 1783-84 to Italy where he purchased classical sculptures that are today in the Gustav III's museum of Antiquities of Sweden. He also visited the stone industry in Florence. Most likely this visit inspired him to support the establishment of porphyry manufacturing operations in Älvdalen (Sweden) with the help of Swedish scientists, such as the naturist Carolus Linnæus (1707-1778) and the mineralogist Axel Fredrik Cronstedt (1722-1765). A huge grinding house was built in 1796-97, driven by waterpower to facilitate porphyry production [31].

\subsection{Late Modern Period}

The Industrial Revolution marked a milestone in the use of building stones. The invention of the steam engine, in addition to the invention of new scientific instruments [171] at the end of the 18th century, led to a spectacular increase in the construction of factories and generalised the consumption of all kinds of products, services and natural resources. On one hand, this economic boom encouraged the founding of the most important societies and museums (Table 3). On the other hand, improvements in transportation and communication routes made it easier for travellers with cultural concerns and merchandise to travel further and further.

Table 3. First main museums.

\begin{tabular}{|c|c|c|}
\hline Year & City-Country & Name Translated into English \\
\hline $1568-1571$ & Munich-Germany & Antiquarium of Munich Residence \\
\hline 1671 & Basel-Switzerland & Amerbach Cabinet \\
\hline 1683 & Oxfort-England & $\begin{array}{l}\text { Ashmolean Museum of Art and } \\
\text { Archaeology }\end{array}$ \\
\hline 1694 & Besancon-France & Museum of Fine Arts and Archeology \\
\hline 1727 & Saint Petersburg-Russia & $\begin{array}{l}\text { The Kunstkammer (Museum of } \\
\text { Anthropology and Ethnography) }\end{array}$ \\
\hline 1734 & Rome-Italy & Capitoline Museums \\
\hline 1740 & Vatican & $\begin{array}{c}\text { Reorganization the Sacred and Profane } \\
\text { Museums (Vatican) }\end{array}$ \\
\hline 1758 & Naples-Italy & Herculanense Museum \\
\hline 1759 & London-England & British Museum \\
\hline 1763 & Madrid-Spain & Antique Cabinet \\
\hline 1764 & Saint Petersburg-Russia & State Hermitage Museum \\
\hline 1769 & Florence-Italy & Uffizi Gallery \\
\hline 1769-1799 & Vatican & Vatican Museums \\
\hline 1773 & Charleston-USA & Charleston Museum \\
\hline 1777 & Naples-Italy & $\begin{array}{c}\text { National Archaeological Museum of } \\
\text { Naples }\end{array}$ \\
\hline 1784 & Haarlem-The Netherlands & Teylers Museum \\
\hline 1793 & París-France & Louvre Museum \\
\hline 1814 & Calcutta-India & Calcutta Museum \\
\hline 1819 & Madrid-Spain & Prado Museum \\
\hline 1848 & Warrington-England & Warrington Museum \\
\hline
\end{tabular}

\subsubsection{Pioneers of Archaeology as a Science}

The main precursors of heritage stone research are shown in Figures 11-17. The Englishman Richard Pococke (1704-1765) travelled to Egypt, Palestine, Jerusalem, Baalbek, Syria and Mesopotamia. He wrote and published A Description of the East and Some other Countries in 1743 [172]. This author was a forerunner of anthropologists and Egyptologists. Later, the military campaign of Egypt and Syria (1798-1801) carried out by the French general Napoleon Bonaparte (1769-1821) also had scientific purposes and constituted the first systematic exploration of Egypt, discovering the Rosetta Stone in 1799. Description de l'Égypte (Description of Egypt) was published between 1809 and 1822, in 24 volumes [173]. The Italians Bernardino Drovetti (1776-1852) and Giovanni Battista Belzoni (1778-1823), together with the English Henry Salt (1780-1827), were the first expedition members to 
collect Egyptian antiquities for collectors and museums such as the Louvre museum, the British museum, the Berlin museum, and the Egyptian museum of Turin.

(a)

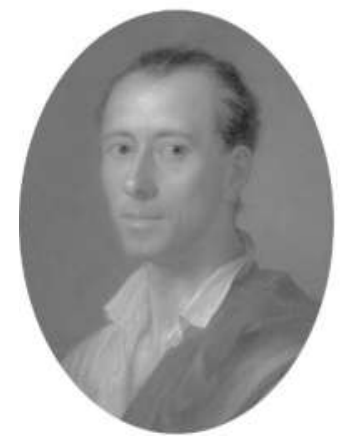

(b)

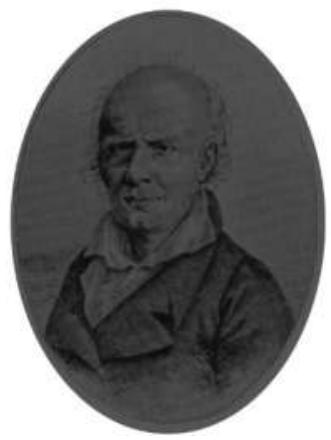

(c)

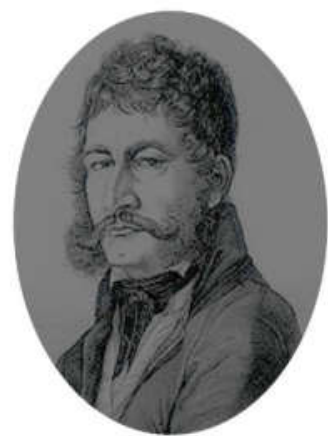

(d)

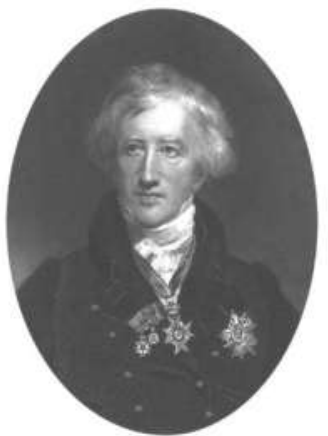

(e)

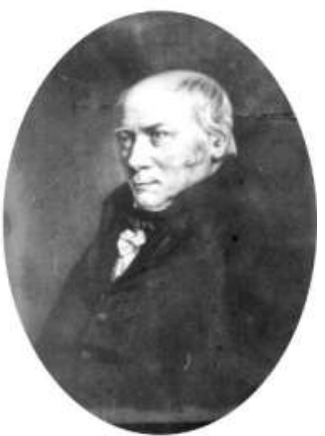

Figure 11. (a) Johann Joachim Winckelmann (1717-1768); (b) Jean-Baptiste Rondelet (1743-1829); (c) Bernardino Drovetti (1776-1852); (d) Baron Cuvier (1769-1832); (e) William Smith (1769-1839).

(a)

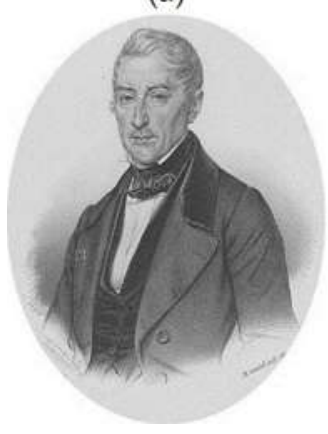

(b)

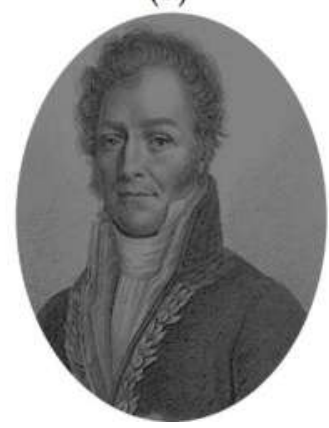

(c)

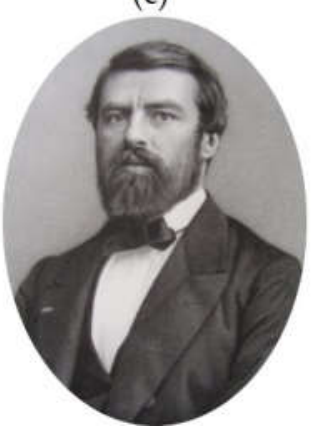

(d)

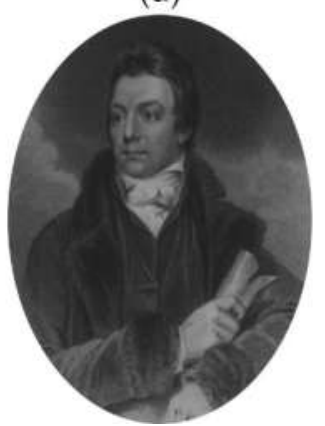

(e)

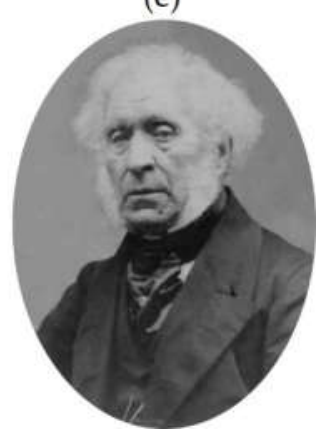

Figure 12. (a) Louis-Étienne François Héricart-Ferrand, vicomte de Thury (1776-1854); (b) Louis Jacques Thénard (17771857); (c) Giovanni Battista Belzoni (1778-1823); (d) Henry Salt (1780-1827); (e) David Brewster (1781-1868).

(a)

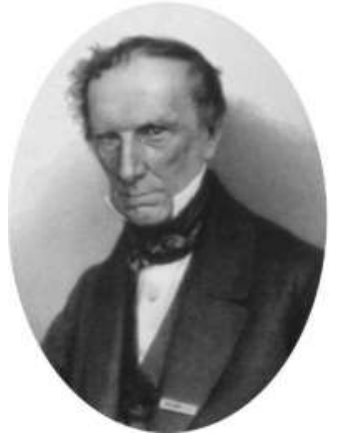

(b)

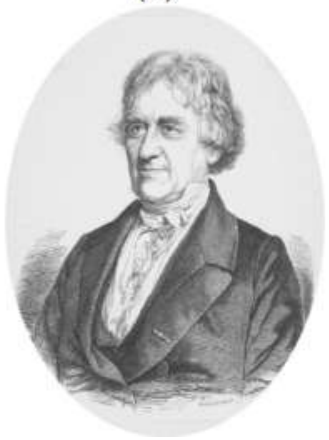

(c)

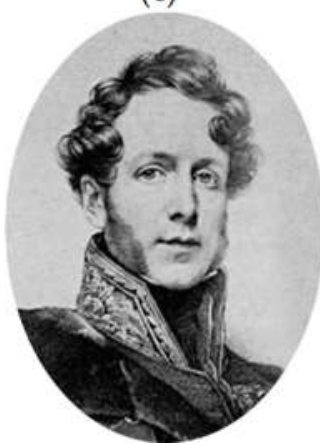

(d)

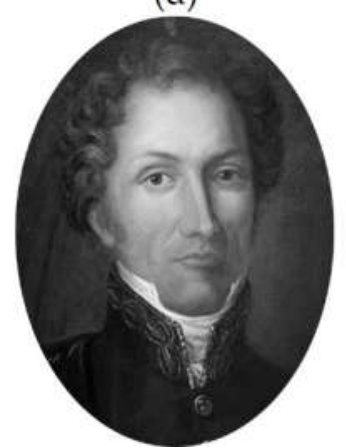

(e)

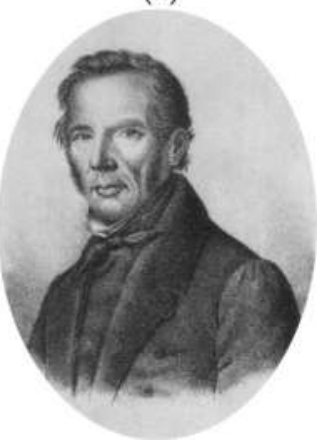

Figure 13. (a) Giovanni Battista Amici (1786-1863); (b) Christian Jürgensen Thomsen (1788-1865); (c) Jacques Boucher de Perthes (1788-1868); (d) Johan Gustaf Liljegren (1791-1837); (e) Carl Georg Brunius (1793-1869). 
(a)

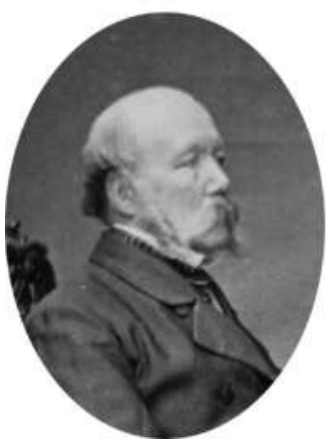

(b)

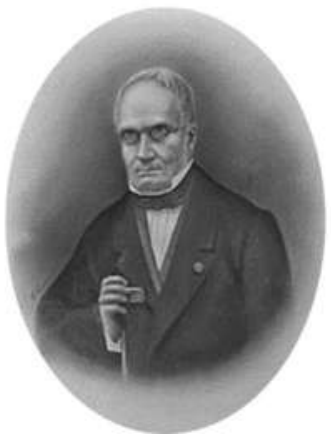

(c)

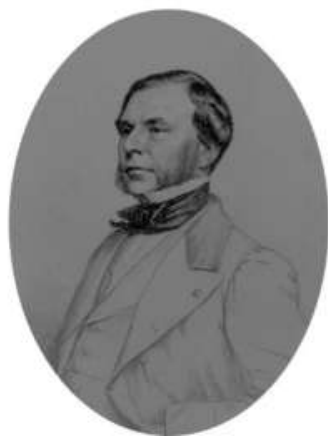

(d)

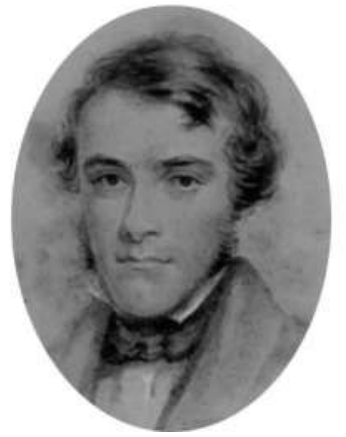

(e)

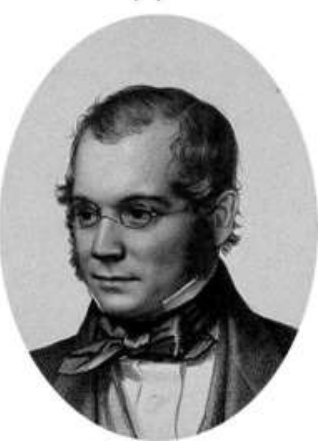

Figure 14. (a) John Gardner Wilkinson (1797-1875); (b) Edouard Lartet (1801-1871); (c) Léon Vaudoyer (1803-1872); (d) Henry Christy (1810-1865); (e) David Thomas Ansted (1814-1880).

(a)

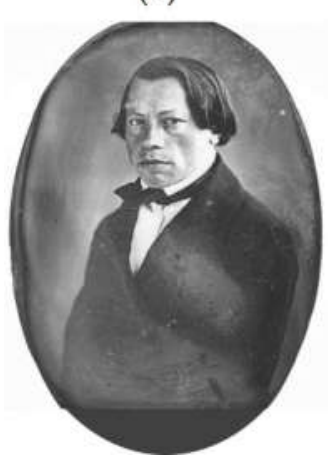

(b)

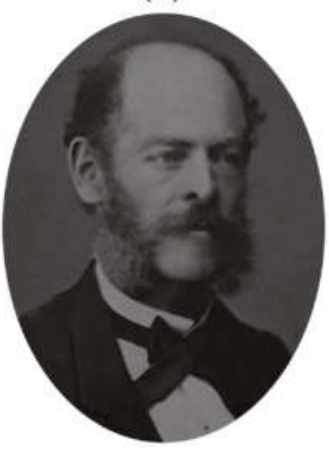

(c)

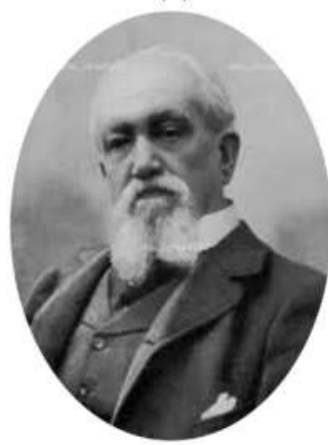

(d)

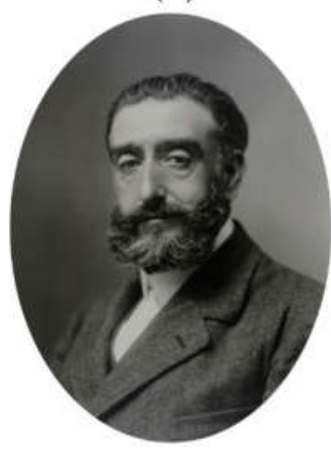

(e)

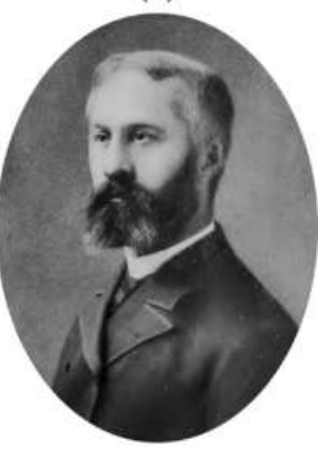

Figure 15. (a) Johannes Menge (1826-1852); (b) Marcelino Sanz de Sautuola (1831-1888); (c) Camilo Boito (1836-1914); (d) José Villa-Amil y Castro (1838-1910); (e) Alexis A. Julien (1840-1919).

(a)

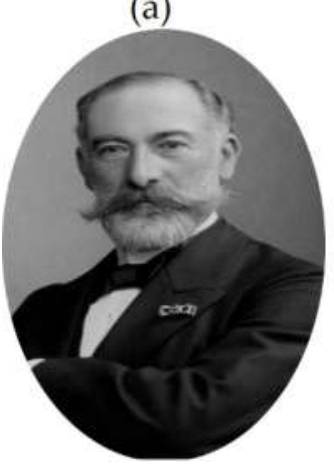

(b)

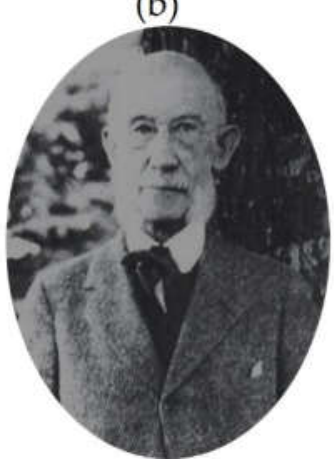

(c)

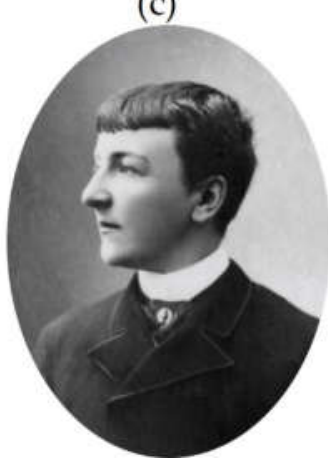

(d)

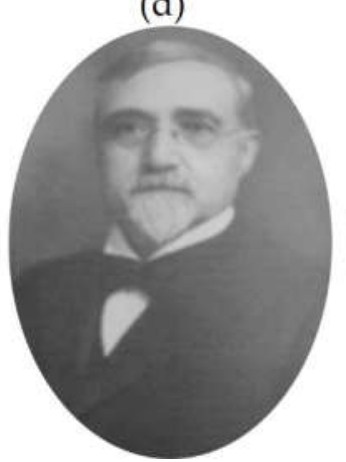

(e)

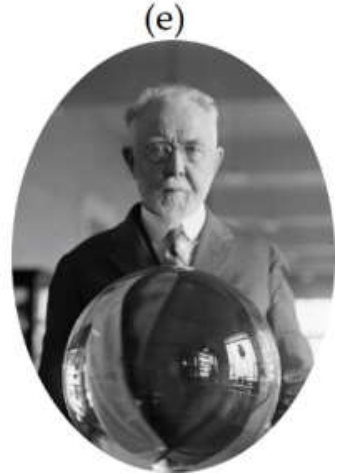

Figure 16. (a) Julius Hirschwald (1845-1928); (b) T. Nelson Dale (1841-1937); (c) Jane Dieulafoy (1851-1916); (d) Ira Osborn Baker (1853-1925); (e) George Perkins Merrill (1854-1929). 
(a)

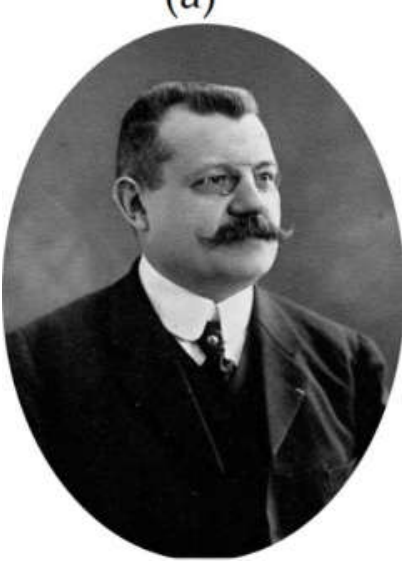

(b)

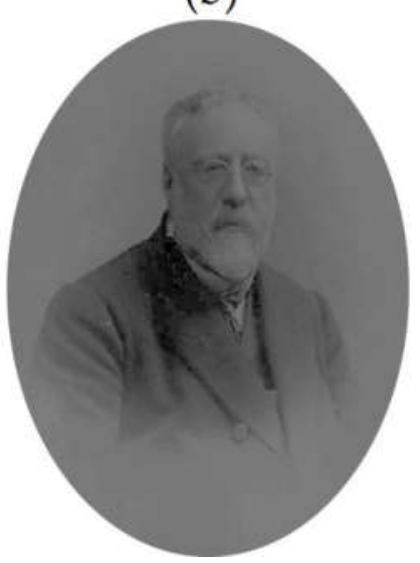

(c)

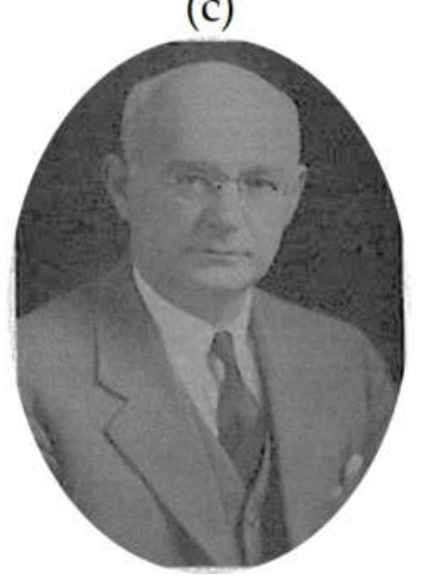

(d)

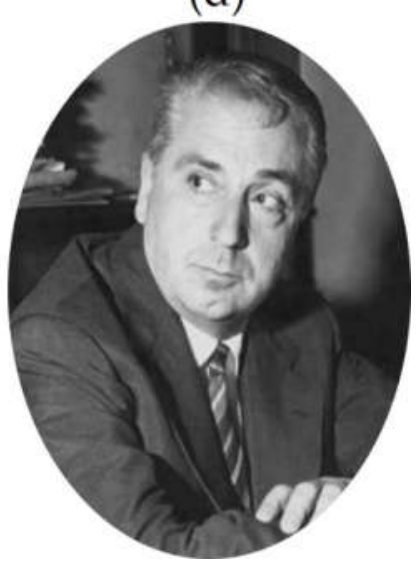

Figure 17. (a) Joseph Déchelette (1862-1914); (b) Hernández-Pacheco y Esteban (1872-1965); (c) Gerald Francis Loughlin (1880-1946); (d) Cesare Brandi (1906-1988).

Archaeology was starting to be a science, and the German archaeologist and art historian Johann Joachim Winckelmann (1717-1768), was one of the founders of history of art and archaeology as a modern discipline. He published Gedanken über die Nachahmung der griechischen Werke in der Malerei und Bildhauerkunst (Reflections on the Imitation of Greek Works in Painting and Sculpture) in 1755 [174]. The Swedish art historian, archaeologist and architect Carl Georg Brunius (1793-1869), together with professor Johan Gustaf Liljegren (1791-1837), wrote the first part of Nordiska Fornlemningar (Nordic Antiquities) published in 1819 as well as a work on petroglyphs [175]. Brunius later produced the first systematic investigation of medieval art and architecture in current Sweden, writing substantial works on the history of art in Scania and in the island of Gotland.

The Danish archaeologist Christian Jürgensen Thomsen (1788-1865) established the system of the three ages (Stone, Bronze and Iron Age), emphasizing the importance of stones in the development of humanity. His results were published in Ledetraad til Nordisk Oldkyndighed (Guide to Scandinavian Antiquity) in 1836 [176]. The excavation of a burial mound in 1837 served to corroborate his hypothesis. At the same time, the English Sir John Gardner Wilkinson (1797-1875) published Manners and Customs of the Ancient Egyptians in three volumes, an exhaustive study collecting twelve years of works in Egypt and Nubia [177].

The French Louis-Jacques-Mandé Daguerre (1787-1851) patented the daguerreotype (the first photographic machine) in 1839. From then on, photographic cameras were perfected, creating stereoscopic cameras for three-dimensional visualization, widely used to photograph monuments. From this discovery, the evolution of important stone-built monuments has been recorded [178]. The French geologist Jacques Boucher de Perthes (1788-1868) began to write Antiquités celtiques et antediluviennes (Celtic and Antediluvian Antiquities) in 1847. He was one of the first persons to postulate the existence of the human being during the final stage of the Pleistocene. His work and findings were not ratified until years later by Jean Paul Rigollot in 1855 and Joseph Prestwich in 1859. The Spanish historian José Villa-Amil y Castro (1838-1910) relates geology with archaeological findings in his work entitled Antigüedades Prehistóricas y Célticas de Galicia (Prehistoric and Celtic Antiquities of Galicia) (1873) [179]. The French archaeologist, novelist, journalist and photographer Jane Dieulafoy (1851-1916), together with her husband, began the first French excavations in Susa (Persia) between 1885 and 1886, and they documented their discoveries with photographs. The French geologist and prehistorian Edouard Lartet (1801-1871) and the English archaeologist Henry Christy (1810-1865) published Reliquiae Aquitanicae. Being Contributions to the Archaeology and Palaeontology of Périgord and the Adjoining Provinces of Southern France in 1866 [180], a description of the portable art that was known in France at that time. The French archaeologist Joseph Déchelette (1862-1914) was one of the 
forerunners of ancient ceramology and published the Manuel D'archéologie Préhistorique, Celtique et Gallo-Romaine (Handbook of Prehistoric, Celtic and Gallo-Roman Archeology) in 1908 [181]. The Spanish naturalist and prehistoric Marcelino Sanz de Sautuola (1831-1888) published Breves apuntes sobre algunos objetos prehistóricos de la provincia de Santander (Brief Notes on Some Prehistoric Objects in the Province of Santander) (1879), where he described the findings of parietal art in the Altamira cave (Spain) and the Spanish geologist Eduardo Hernández-Pacheco y Esteban (1872-1965) published The Prehistoric Paintings of Peña Tu (Asturias) in 1914 [182]. This geologist delved into the study of prehistoric heritage and natural monuments throughout his long career [183-186].

\subsubsection{Beginnings in Restoration}

The restoration of buildings and conservation of heritage stones, as we know today, was also born in the contemporary age. An interest in the restoration of medieval buildings developed in France in the early 1830s. Three main theories of restoration existed. William Morris (1834-1896) argued that restoration damages the buildings. He defined restoration as "stripping from a building". Anything which looks artistic is worth protecting, so he argued for "Protection" instead of "Restoration". John Ruskin (1819-1900) argued that it is not possible to restore a building and reach its most beautiful version because the eye of the original workman who built the monument cannot be mimicked. He defended that if the buildings are cared for, they do not need to be restored. He thought that the buildings belong to the person who built them, and restorers do not have the right to intervene. Another important architect was Eugène Viollet-le-Duc (1814-1879). He thought in a different way than the other two and defended restoration. He explains restoration as re-establishing a building in a finished state. Viollet-le-Duc said that the architect can make some additions to the building according to the era that he is restoring. This architect restored the Romanesque abbey of Vézelay, Notre Dame de Paris and various buildings in Mont Saint-Michel, Carcassonne, Roquetaillade and Pierrefonds (France).

The Italian architect Camilo Boito (1836-1914), wrote the first restoration letter at the Third Congress of Italian Engineers and Architects in 1883, and around this time the "Opificio delle Pietre Dure" of Florence stopped the production of works of art and began the study of stone restoration. The Italian architect Gustavo Giovannoni (1873-1947) proposed the "Restoration Theory" in 1912. Giovannoni promoted the consolidation and maintenance works using modern techniques. He rejected reconstruction and admitted so-called renovation restorations.

The First World War (1914-1918) caused damage to the towns closest to the battlefields; the use of long-range cannons ruined much of the minor architecture, church towers and supply areas of the armed forces. After the First World War the value of minor architecture was recognized and with it a heritage was identified in cities and villages. Post-war reconstruction practices led to specific interventions, in which the restoration had to be carried out in identical conditions to the original through procedures such as anastylosis. That is, the re-composition of existing but dismembered parts. This principle was very favourable because it restored the image of buildings and towns to the one they had before the war. A new current of thought, exemplified by Gustavo Giovannoni, began to propose that urban settlements contained, like architecture, use and museum values, which led to the inclusion of the concepts of urban heritage and protection of historic city centers during their reconstruction. Gustavo Giovannoni headed the Italian delegation in 1931 at the First International Congress of Architects and Technicians of Historic Monuments in Athens, spreading the doctrines of Camilo Boito, and was met with great reception. The Athens Charter for the Restoration of Historic Monuments was accepted internationally in 1931, and from this time on, a debate was created about how to conduct restorations and what materials to use. The Charter of Athens, as the first international document related to the protection of ancient structures and monuments highlighted the necessity of the production of inventories and cataloguing of goods that enriched the history of Europe. The Italian Restoration Charter was drawn up in 1932 by the Consiglio Superiore per le 
Antichità e Belle Arti (Superior Council of Antiquities and Fine Arts), and set limits on reconstructions, recommending conservation work over restoration work.

The Second World War (1939-1945) paralyzed research on building materials, and scientific research was mainly focused on the War [187]. It seriously damaged the European Built Heritage, aerial bombardments destroyed entire cities. The reconstructions were done mainly with natural stone and Portland cement. To obtain natural stone, historical quarries were exploited, and new ones were opened. After the War, UNESCO (United Nations Educational, Scientific and Cultural Organization) was founded in 1945.

The Italian Institute Centrale del Restauro (ICR) was created in 1939. Its first director, the art historian Cesare Brandi (1906-1988), initiated a work methodology like that of the Fogg Art museum Restoration Laboratory in United States of America, beginning to study heritage from a more scientific point of view.

The First Congress of Architects and Specialists of Historic Buildings (Paris, 1957) recommended that the countries lacking a central organisation for the protection of historic buildings provide for the establishment of such an authority. In addition, that all member join the International Centre for the Study of the Preservation and Restoration of Cultural Property (ICCROM), based in Rome.

The Second Congress of Architects and Specialists of Historic Buildings (Venice, 1964) adopted several resolutions, the first one being the International Restoration Charter, better known as the Venice Charter, and the second one, put forward by UNESCO, provided for the creation of the International Council on Monuments and Sites (ICOMOS-ISCS).

Concepts such as "systematic maintenance" were introduced as a fundamental operation for the conservation and "in situ conservation" of excavated remains. It also excluded "a priori, any reconstruction work", accepted only anastylosis, and urged that the material used for the intervention should be fully recognisable.

The Italian Charter for the Restoration of Historic Monuments or "Carta del Restauro", signed in 1972 [188] defined the concepts of conservation, restoration, safeguarding and reversibility, as well as giving precise instructions for the custody of heritage. That same year, the UNESCO convention for the protection of world heritage was signed, which defined the kind of natural or cultural sites which can be considered for inscription on the World Heritage List.

The European Charter of Architectural Historical Heritage (Amsterdam Charter) was signed in 1975, which states: "The European architectural heritage consists not only of our most important monuments: it also includes the groups of lesser buildings in our old towns and characteristic villages in their natural or manmade settings" [189].

\subsubsection{Pioneers in Scientific Research on Building Stones}

Regarding the investigation on properties of building materials, the French architects Jean-Baptiste Rondelet (1743-1829) and Léon Vaudoyer (1803-1873) calculated the mechanical resistance and water absorption respectively of the most used building stones in the late 18th and early 19th centuries. David Brewster (1781-1868) was a British scientist and inventor who worked in physical optics, studied the polarisation of light, and discovered Brewster's angle. Contemporarily, Scottish physicist and geologist William Nicol (1768-1851) developed a method for preparing thin sections for microscopical study in 1815. Nicol also invented the prism that bears his name in 1828 . The first fully polarising microscope was built in 1830 by the Italian astronomer and optician Giovanni Battista Amici (1786-1863). These scientific-technological advances were fundamental for the scientific study of construction materials as it is understood nowadays.

The French naturalist Baron Cuvier (1769-1832) published between 1816 and 1845, 61 volumes of the Dictionnaire des Sciences Naturelles (Dictionary of Natural Sciences) with important contributions to the subject of building materials, such as the description of the making of bricks (Volume II), in addition to other books on geology. The Italian archivist and politician Luigi Bossi Visconti (1758-1835) published Dizionario Portatile di Geologia, Litologia e Mineralogia (Portable Dictionary of Geology, Lithology and Mineralogy) [190] in 
1819, and James Mitchell published A Dictionary of Chemistry, Mineralogy, and Geology: in Accordance with the Present State of those Sciences in 1823 [191].

The French mining engineer Louis-Étienne Héricart de Thury (1776-1854) developed frost durability tests in 1828 [192-194], and the French chemist Louis Jacques Thénard (1777-1857) conducted tests of durability on plaster bas-reliefs to study their dissolution.

The English geologist William Smith (1769-1839) was part of a commission to select replacement ashlars in the restoration of the Palace of Westminster in London in 1838 [195] where the durability of the material played a fundamental role in the choice of the stones. Around this time, the English were also exploring their South Pacific colonies. The planners of colonial South Australia appreciated the important potential of building stone resources, as evidenced by the appointment of the German Johannes Menge (1788-1852) as Geologist for the South Australian Company [196].

Portland cement was invented by the English builder Joseph Aspdin (1778-1855) in 1824. This invention, together with the development of the internal combustion engine, electric power and railway lines would revolutionise the trade and export of natural stone in the second half of 19th century [197]. Tests were frequently conducted prior to the opening of new quarries to evaluate the quality of construction stones [198]. The English professor of geology David Thomas. Ansted (1814-1880) published On the Decay and Preservation of Building Materials in 1860 [199], where he pointed out that all stones are altered or weathered on the topmost part of quarries or near the ground surface and studied the alteration of potassium feldspars.

The German geologist Julius Hirschwald (1845-1928) participated in the commission for the determination of a method for the study of the resistance of rocks against atmospheric phenomena in 1893 and defined a coefficient based on the saturation kinetics of stones for the evaluation of their resistance to frost in 1908 [200]. He also published a guide to laboratory tests of building materials for engineers, architects and stonemasons in 1912. The English geologist John Allen Howe (1876-1959) published The Geology of Building Stones in 1910 [201] and Stones and Quarries in 1920 [202].

The International Union of Laboratories and Experts in Building Materials, Systems and Structures (RILEM, from its name in French) was created with the aim of promoting scientific cooperation in building materials and structures in 1947.

\subsubsection{First Research in the United States of America}

The Brownstone industry for the construction of buildings, monuments and stone walls in the United States developed in the 18th and 19th centuries. Brownstone is a brown Triassic-Jurassic sandstone used as building stone. There are five main types: Apostle Island brownstone from Basswood Island (Wisconsin); Hummelstown brownstone from Hummelstown, Pennsylvania. It was very popular along the East Coast of the United States. Delaware, New York, Maryland, Pennsylvania and West Virginia government buildings were faced entirely with this stone; Portland brownstone, from Portland, Connecticut, and nearby localities were used in a number of landmark buildings in Baltimore, Boston, Chicago, New York City, New Haven, Hartford, Philadelphia and Washington D.C.; New Jersey brownstone from the Passaic Formation in northern New Jersey supplied mainly New York City and the state of New Jersey; and South Wales brownstone was commonly used in Southern Wales [203].

The development in building stone research by North American scientists from the late nineteenth to the mid-twentieth century is noteworthy. The selection of the stone for the Lincoln Memorial was entrusted to the American geologist George Perkins Merrill (1854-1929), who published On the Collection of Maine Building Stones in the United States National Museum in 1883 [204]. The same author published the book entitled The Collection of Building and Ornamental Stones in the United States National museum in 1889 [205]. He also published Stones for Building and Decoration in 1891 [206] and Principles of Rock Weathering, an important article on the principles of decay in 1896. In 1897 he published A treatise on rocks, rock-weathering and soils [207]. The also American geologist Alexis A. 
Julien (1840-1919) wrote The Durability of Building Stones in New York City and Vicinity in 1884 [208].

The University of Illinois professor of civil engineering Ira Osborn Baker (1853-1925) published A Treatise on Masonry Construction in 1890 [209], where the durability of construction materials figures prominently. The geologist George Wesson Hawes (1848-1882) started a collection of building stones for the National museum in Washington D.C, (now the Smithsonian Institution's National museum of Natural History), where petrophysical and petrological studies were carried out. Additionally, in the United States of America, the "National Bureau of Standards" (NBS) was founded in 1901, which began the study of building stones in 1912, with the publishing of many scientific articles in "Technological Papers". The American geologist Gerald Francis Loughlin (1880-1946) wrote his thesis on Boston building stones in 1903 and a year later, together with his colleague William Otis Crosby (1850-1925), they published an article on the subject [210]. That same year, the American geologist, T. Nelson Dale (1841-1937) published an important work on the construction granite of Massachusetts, New Hampshire, and Rhode Island [211] and on New England building granite with studies of quarrying and durability in 1923 [212]. The American geologist Edwin Clarence Eckel (1875-1941) published Building Stones and Clays in 1912 [213].

The American Daniel W. Kessler, of the National Bureau of Standards (Figure 18), published a treatise on physical and chemical tests on building materials in 1919 [214]. In 1927, he also published a book on the physical properties of the main commercial limestones used for the construction of buildings in the United States of America [215]. Some of this work culminated in the publication of several American Society for Testing and Materials (ASTM) standards on dimension stones which are still in use today. Mr. Kessler was honored by ASTM in 1950 with the ASTM Award of Merit. His compatriot, the American geologist Stephen Taber published works on the mechanisms of ice crystallization in stones in 1929 and 1930 [216,217], and the American Robert John Schaffer published Weathering of Natural Building Stones in 1932 [218].
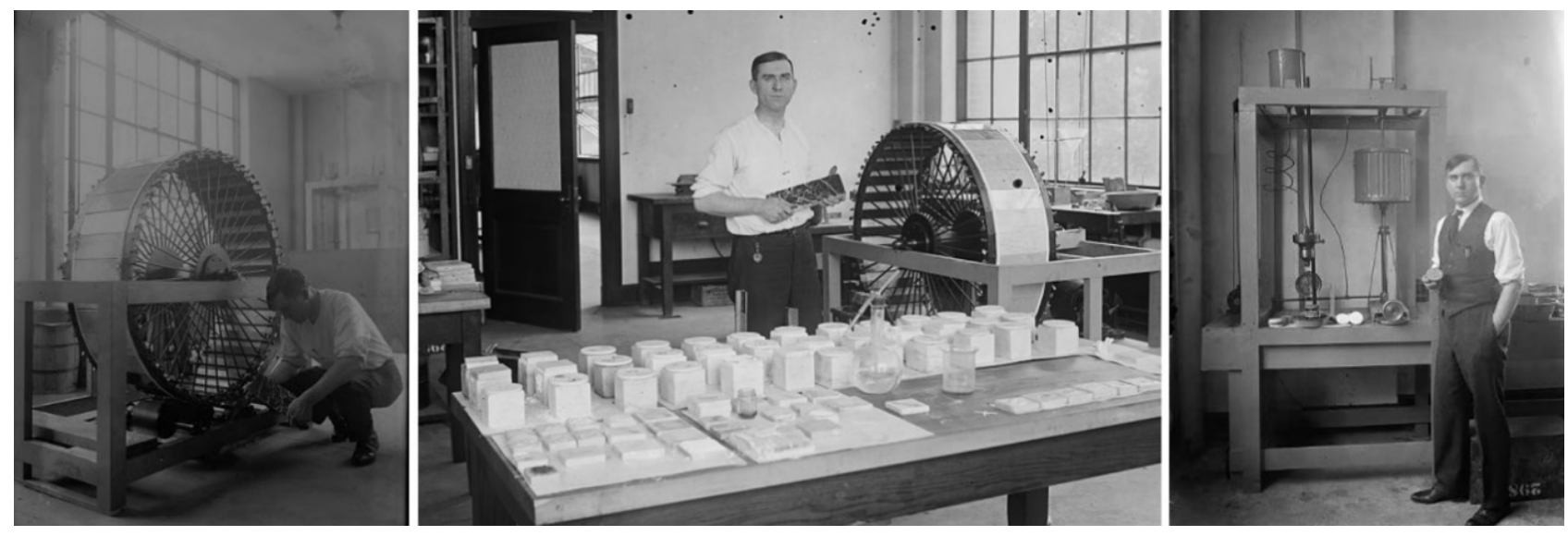

Figure 18. Daniel W. Kessler, 1925. National Bureau of Standards (USA).

\section{Recent Works}

Erhard M. Winkler (1921-2005), professor of University of Notre Dame (Indiana, USA) wrote in 1973 Stone. Properties, Durability in Man's Environment, and Stone in Architecture Properties, Durability in 1997, among other publications [219-222]. In addition, Joseth T. Hannibal published many books on the subject about building stones from the 1980s onwards [223,224] and Doehne and Price [225] published a magnificent compilation of articles on stone conservation in 2010. The past few decades has seen an unprecedented level of research activity in this area, the results of which are summarized in the latest edition of Stone in Architecture Properties, Durability by Siegfried Siegesmund and Rolf 
Snethlage [226]. It is a currently reference book in this field and it is a revision of the first editions by Erhard M. Winkler.

In this time, there is a growing interest in the investigation of deterioration and conservation of building stones [227-234]. The development of analytical techniques allows scientists to quickly obtain increasingly reliable results and non-destructive and portable diagnostic techniques have improved considerably and play an important role in the conservation of built heritage [235-237]. However, we scientists must not forget that each heritage stone is a remains of the past, unique and unrepeatable. The traditional techniques and the conditions in which the heritage stones have been extracted from the historical quarry, carved and placed in the monument are key to understanding its deterioration and conservation. The challenges of heritage stone research in the 21st century are: climate change mitigation, monitoring, remote sensing [238-241], adaptation [242], restoring after conflicts, disasters, or pandemics, the achievement of sustainable development goals [243249], and most importantly, the preservation of a legacy for the future.

Funding: This study was supported by Portuguese funds by FUNDAÇÃO PARA A CIÊNCIA E A TECNOLOGIA, I.P. (PORTUGAL) in the frame of the UIDB/00073/2020 project of the I \& D unit Geosciences Center (CGEO) and Stimulus of Scientific Employment, Individual Support 2017. CEECIND/03568/2017.

Institutional Review Board Statement: Not applicable.

Informed Consent Statement: Not applicable.

Conflicts of Interest: The authors declare no conflict of interest.

\section{References}

1. Gori, S.; Falcucci, E.; Galadini, F.; Moro, M.; Saroli, M.; Ceccaroni, E. Geoarchaeology and paleoseismology blend to define the Fucino active normal fault slip history, central Italy. Quat. Int. 2017, 451, 114-128. [CrossRef]

2. Smith, B.D.; Zeder, M.A. The onset of the Anthropocene. Anthropocene 2013, 4, 8-13. [CrossRef]

3. Finney, S.C. The 'Anthropocene' as a ratified unit in the ICS International Chronostratigraphic Chart: Fundamental issues that must be addressed by the Task Group. In A Stratigraphical Basis for the Anthropocene; Waters, C.N., Zalasiewic, J.A., Williams, M., Ellis, M.A., Snelling, A.M., Eds.; Geological Society: London, UK, 2014; Volume 395, pp. 23-28. [CrossRef]

4. Ruddiman, W.F.; Ellis, E.C.; Kaplan, J.O.; Fuller, D.Q. Defining the epoch we live in. Science 2015, 348, 38-39. [CrossRef] [PubMed]

5. Knutsson, H.; Knutsson, K.; Molin, F.; Zetterlun, P. From flint to quartz: Organization of lithic technology in relation to raw material availability during the pioneer process of Scandinavia. Quat. Int. 2016, 424, 32-57. [CrossRef]

6. Banegas, A.; Goye, M.S. Spatial and temporal variability in the use of lithic raw materials for flaked stone technology in northeast Chubut Province (North Patagonia) during the Late Holocene. Quat. Int. 2015, 373, 55-62. [CrossRef]

7. Garrido Cordero, J.A. El uso del cuarzo y el cristal de roca en la prehistoria reciente andaluza. Estado de la cuestión y análisis de un fenómeno cultural. Rev. Atl. Mediterr. 2015, 17, 187-200. [CrossRef]

8. Watchman, A.; Taçon, P.; Aubert, M. Correspondence on "Erosion rates and weathering history of rock surfaces associated with Aboriginal rock art engravings (petroglyphs). In Burrup Peninsula, Western Australia, from cosmogenic nuclide measurements by Brad Pillans and Keith Fifield. Quat. Sci. Rev. 2014, 69, 98-106. [CrossRef]

9. Fauzi, M.R.; Intan, F.S.; Wibowo, A.S. Newly discovered cave art sites from Bukit Bulan, Sumatra: Aligning prehistoric symbolic behavior in Indonesian prehistory. J. Archaeol. Sci. Rep. 2019, 24, 166-174. [CrossRef]

10. Loendorf, C.; Blikre, L.; Bryce, W.D.; Oliver, T.J.; Denoyer, A.; Wermers, G. Raw material impact strength and flaked stone projectile point performance. J. Archaeol. Sci. 2018, 90, 50-61. [CrossRef]

11. Harper, K.; McCormick, M.; Hamilton, M.; Peiffert, C.; Michels, R.; Engel, M. Establishing the provenance of the Nazareth Inscription: Using stable isotopes to resolve a historic controversy and trace ancient marble production. J. Archaeol. Sci. Rep. 2020, 30, 102228. [CrossRef]

12. Johnson, K.M.; Ouimet, W.B. Physical properties and spatial controls of stone walls in the northeastern USA: Implications for Anthropocene studies of 17th to early 20th century agriculture. Anthropocene 2016, 15, 22-36. [CrossRef]

13. Autelitano, F.; Garilli, E.; Giuliani, F. Criteria for the selection and design of joints for street pavements in natural stone. Constr. Build. Mater. 2020, 259, 119722. [CrossRef]

14. Siegesmund, S.; Török, A. Building stones. In Stone in Architecture—Properties, Durability, 4th ed.; Siegesmund, S., Snethlage, R., Eds.; Springer: Berlin/Heidelberg, Germany, 2011; pp. 11-96.

15. Elsen, J.; Brutsaert, A.; Deckers, M.; Brulet, R. Microscopical study of ancient mortars from Tournai (Belgium). Mater. Charact. 2004, 53, 289-294. [CrossRef] 
16. La Russa, M.F.; Rovella, N.; Pelosi, C.; Rossi, D.; Benucci, M.; Romagnoli, G.; Selva Bonino, V.E.; Casoli, A.; Ruffolo, S.A. A multianalytical approach applied to the archaeometric study of mortars from the Forty Martyrs rupestrian complex in Cappadocia (Turkey). Microchem. J. 2016, 125, 34-42. [CrossRef]

17. Delgado Rodrigues, J.; Costa, D. A new interpretation methodology for microdrilling data from soft mortars. J. Cult. Herit. 2016, 22, 951-955. [CrossRef]

18. Kay, A.U.; Kaplan, J.O. Human subsistence and land use in sub-Saharan Africa, 1000 BC to AD 1500: A review, quantification, and classification. Anthropocene 2015, 9, 14-32. [CrossRef]

19. Mendes, M.T.; Esteves, L.; Ferreira, T.A.; Candeias, A.; Tennent, N.H.; Delgado Rodrigues, J.; Pereira, S. Lacunae infills for in situ treatment of historic glazed tiles. Appl. Phys. A 2016, 122, 547. [CrossRef]

20. Pérez, N.A.; Bucio, L.; Lima, E.; Soto, E.; Cedillo, C. Identification of allophane and other semi-crystalline and amorphous phases on pre-Hispanic Mexican adobe earth bricks from Cholula, Mexico. Microchem. J. 2016, 126, 349-358. [CrossRef]

21. Martínez, P.; Soto, M.; Zunino, F.; Stuckrath, C.; Lopez, M. Effectiveness of tetra-ethyl-ortho-silicate (TEOS) consolidation of fired-clay bricks manufactured with different calcination temperatures. Constr. Build. Mater. 2016, 106, 209-217. [CrossRef]

22. Županek, B.; Lesar Kikelj, M.; Žagar, M.; Kramar, S. A new lightweight support for the restoration and presentation of a large Roman mosaic. J. Cult. Herit. 2016, 19, 477-485. [CrossRef]

23. Erlandson, J.; Braje, T.J. Archeology and the Anthropocene. Anthropocene 2013, 4, 1-7. [CrossRef]

24. Myriounis, C.; Varras, G.; Tsirogiannis, I.; Pavlidis, V. Usage of stone materials in natural and human environment, case study in Epirus, Greece. Agric. Agric. Sci. Procedia 2015, 4, 431-439. [CrossRef]

25. Uchida, E.; Shimoda, I. Quarries and transportation routes of Angkor monument sandstone blocks. J. Archaeol. Sci. 2013, 40, 1158-1164. [CrossRef]

26. Galetakis, M.; Soultana, A. A review on the utilisation of quarry and ornamental stone industry fine by-products in the construction sector. Constr. Build. Mater. 2016, 102, 769-781. [CrossRef]

27. Darras, V.; Mireles, C.; Siebe, C.; Quezada, O.; Castañeda, A.; Reyes-Guzmán, N. The other stone. Dacite quarries and workshops in the prehispanic Tarascan territory, Michoacán, Mexico. J. Archaeol. Sci. Rep. 2017, 12, 219-231. [CrossRef]

28. Harrell, J.A. Ancient Egyptian limestone quarries: A petrological survey. Archaeometry 1992, 34, 195-212. [CrossRef]

29. De Laet, V.; van Loon, G.; van der Perre, A.; Deliver, I.; Willems, H. Integrated remote sensing investigations of ancient quarries and road systems in the Greater Dayr al-Barsha Region, Middle Egypt: A study of logistics. J. Archaeol. Sci. 2015, 55, 286-300. [CrossRef]

30. Quinn, T. About Museums, Culture, and Justice to Explore in Your Classroom, 1st ed.; Ayers, W., Ed.; Columbia University: New York, NY, USA, 2020; p. 95.

31. Wikström, A.; Pereira, D.; Lundquvist, T.; Cooper, B. The Dala (Älvdalen) porphyries from Sweden. Episodes 2015, 38, 79-84. [CrossRef]

32. Bulakh, A. "Porphyries" from Russia and Sweden used in St Petersburg and Russian "porphyry" used in Paris: Misuse of a geological term for some possible candidate as a global Heritage Stone Resource. Episodes 2015, 38, 114-117. [CrossRef]

33. Pereira, D.; Tourneur, F.; Bernáldez, L.; García Blázquez, A. Petit Granit: A Belgian limestone used in heritage, construction and sculpture. Episodes 2015, 38, 85-90. [CrossRef]

34. De Kock, T.; Boone, M.; Dewanckele, J.; De Ceukelaire, M.; Cnudde, V. Lede Stone: A potential "Global Heritage Stone resource" From Belgium. Episodes 2015, 38, 91-96. [CrossRef] [PubMed]

35. Cardenes, V.; Cnudde, V.; Cnudde, J.P. Iberian roofing slate as a global Heritage Stone Province Resource. Episodes 2015, 38, 97-105. [CrossRef]

36. Marker, B.R. Bath Stone and Purbeck Stone: A comparison in terms of criteria for Global Heritage Stone Resource Designation. Episodes 2015, 38, 118-123. [CrossRef] [PubMed]

37. Cooper, B.J.; Branagan, D.F.; Franklin, B.; Ray, H. Sydney sandstone: Proposed "Global Heritage Stone Resource" from Australia. Episodes 2015, 38, 124-131. [CrossRef] [PubMed]

38. Freire-Lista, D.M.; Fort, R. The Piedra Berroqueña region: Candidacy for Global Heritage Stone Province status. Geosci. Can. 2016, 43, 43-52. [CrossRef]

39. Freire-Lista, D.M.; Fort, R.; Varas-Muriel, M.J. Alpedrete granite (Spain). A nomination for the "Global Heritage Stone Resource" designation. Episodes 2015, 38, 106-113. [CrossRef]

40. Freire-Lista, D.M.; Fort, R. Cadalso de los Vidrios leucogranite "Blanco Cristal", a widely used heritage stone. J. Geol. Soc. Lond. Spec. Publ. 2019, 486, 53-65. [CrossRef]

41. Bams, V.; Dewaele, S. Staining of white marble. Mater. Charact. 2007, 58, 1052-1062. [CrossRef]

42. Murru, A.; Freire-Lista, D.M.; Fort, R.; Varas-Muriel, M.J.; Meloni, P. Evaluation of Post-thermal Shock Effects in Carrara Marble and Santa Caterina di Pittinuri limestone. Constr. Build. Mater. 2018, 186, 1200-1211. [CrossRef]

43. Siegesmund, S.; Snethlage, R. Stone in Architecture, 5th ed.; Springer: Berlin/Heidelberg, Germany, 2014; p. 550. [CrossRef]

44. Berge, B. The Ecology of Building Materials, 2nd ed.; Architectural Press: Oxford, UK, 2009; p. 412.

45. Dietz, R.S.; McHone, J. Kaaba Stone: Not A Meteorite, Probably an AGATE. Meteoritics 1974, 9, 173-179. [CrossRef]

46. Thomsen, E. New light on the origin of the holy black stone of the Ka'ba. Meteoritics 1980, 15, 87-91. [CrossRef]

47. Axon, H.J. The black stone of the Ka'ba: Suggestions as to its constitution. J. Mater. Sci. Lett. 1982, 1, 10-12. [CrossRef] 
48. Manrique-Ortega, M.D.; Casanova-González, M.A.; Jiménez-Galindo, E.; Ruvalcaba-Sil, L.A. Methodology for the non-destructive characterization of jadeite-jade for archaeological studies. Spectrochim. Acta Part A 2019, 217, 294-309. [CrossRef] [PubMed]

49. Huang, X.; Chen, M.; Yang, Q.; Zhu, Y.; Wang, K.; Fang, X.; Hu, D. Natural reinforcing effect of inorganic colloids on excavated ancient jades. J. Cult. Herit. 2020, 46, 52-60. [CrossRef]

50. Harlow, G.E.; Berman, M.J.; Cárdenas Párraga, J.; Hertwig, A.; García-Casco, A.; Gnivecki, P.L. Pre-Columbian jadeitite artifacts from San Salvador Island, Bahamas and comparison with jades of the eastern Caribbean and jadeitites of the greater Caribbean region. J. Archaeol. Sci. Rep. 2019, 26, 101830. [CrossRef]

51. Tardy, N.; Vosges, J.; Varoutsikos, B. Micro-blade production on hyaline quartz during the Late Neolithic of northern Greece (5400-4600 cal. B.C.): Examples from Dikili Tash and Promachonas-Topolniča. Quat. Int. 2016, 424, 212-231. [CrossRef]

52. Evans, J.D. The Prehistoric Antiquities of the Maltese islands: A Survey, 1st ed.; Athelone Press of the University of London: London, UK, 1971; p. 404.

53. De Franceschini, M. Equinoctial Orientations in the Mediterranean Sea: The Prehistoric Temple of Mnajdra and the Mausoleo degli Equinozi in Rome. In Proceedings of the Seminario di Archeoastronomia ALSSA, Genova, Italy, $24-25$ March 2012 ; p. 14.

54. Robinson, M.G.P.; Porter, A.; Figueira, W.; Fletcher, R. Neolithic Temples of Malta: 3D analysis points to novel roof reconstruction. Digit. Appl. Archaeol. Cult. Herit. 2019, 13, e00095. [CrossRef]

55. Grøntoft, T.; Cassar, J. An assessment of the contribution of air pollution to the weathering of limestone heritage in Malta. Environ. Earth Sci. 2020, 79, 288. [CrossRef]

56. Bradley, S.; Cummings, V.; Baker, M.J. Sources of flint in Britain and Ireland: A quantitative assessment of geochemical characterisation using acid digestion inductively coupled plasma-mass spectrometry (ICP-MS). J. Archaeol. Sci. Rep. 2020, 31, 102281. [CrossRef]

57. Cartailhac, E. La France Préhistorique. d'Après les Sépultures et les; Alcan, F., Ed.; Ancienne Librairie Germer Baillière: Paris, France, 1889.

58. Lhote, M. Au sujet des haches polies de petites dimensions. Bull. Soc. Préhist. Frangaise 1952, 49, 524-528. [CrossRef]

59. Wadsworth, J.; Lesuer, D.R. Ancient and modern laminated composites Đ from the Great Pyramid of Gizeh to Y2K. Mater. Charact. 2000, 45, 289-313. [CrossRef]

60. Comelli, D.; D’Orazio, M.; Folco, L.; El-Halwagy, M.; Frizzi, T.; Alberti, R.; Capogrosso, V.; Elnaggar, A.; Hassan, H.; Nevin, A.; et al. The meteoritic origin of Tutankhamun's iron dagger blade. Meteorit. Planet. Sci. 2016, 51, 1301-1309. [CrossRef]

61. Finkel, M.; Gopher, A.; Agam, A. Excavating tailing piles at Kakal Spur (Kerem Ben Zimra) locality in the Nahal Dishon prehistoric flint extraction and reduction complex, northern Galilee, Israel. Archaeol. Res. Asia 2020, 23, 100207. [CrossRef]

62. Sánchez Yustos, P.; Diez-Martín, F.; Díaz, I.M.; Duque, J.; Fraile, C.; Domínguez, M. Production and use of percussive stone tools in the Early Stone Age: Experimental approach to the lithic record of Olduvai Gorge, Tanzania. J. Archaeol. Sci. Rep. 2015, 2, 367-383. [CrossRef]

63. Dietrich, L.; Götting-Martin, E.; Hertzog, J.; Schmitt-Kopplin, P.; McGovern, P.E.; Hall, G.R.; Christian Petersen, W.; Zarnkow, M.; Hutzler, M.; Jacob, F.; et al. Investigating the function of Pre-Pottery Neolithic stone troughs from Göbekli Tepe-An integrated approach. J. Archaeol. Sci. Rep. 2020, 34, 102618. [CrossRef]

64. Peña-Chocarro, L.; Pérez-Jordà, G.; Morales, J. Crops of the first farming communities in the Iberian Peninsula. Quat. Int. 2018, 470, 369-382. [CrossRef]

65. Kedar, Y.; Kedar, G.; Barkai, R. Setting fire in a Paleolithic Cave: The influence of cave dimensions on smoke dispersal. J. Archaeol. Sci. Rep. 2020, 29, 102112. [CrossRef]

66. Rosado, L.; Van Pevenage, J.; Vandenabeele, P.; Candeias, A.; Lopes, M.C.; Tavares, D.; Alfenim, R.; Schiavon, N.; Mirão, J. Multi-analytical study of ceramic pigments application in the study of Iron Age decorated pottery from SW Iberia. Measurement 2018, 118, 262-274. [CrossRef]

67. Sotiropoulou, S.; Karapanagiotis, I.; Andrikopoulos, K.S.; Marketou, T.; Birtacha, K.; Marthari, M. Review and New Evidence on the Molluscan Purple Pigment Used in the Early Late Bronze Age Aegean Wall Paintings. Heritage 2021, 4, 171-187. [CrossRef]

68. Izzo, F.; Arizzi, A.; Cappelletti, P.; Cultrone, G.; De Bonis, A.; Germinario, C.; Graziano, S.F.; Grifa, C.; Guarino, V.; Mercurio, M.; et al. The art of building in the Roman period (89 B.C.-79 A.D.): Mortars, plasters and mosaic floors from ancient Stabiae (Naples, Italy). Constr. Build. Mater. 2016, 117, 129-143. [CrossRef]

69. Guilaine, J.; Briois, F.; Vigne, J.D.; Carrère, I. Découverte d'un Néolithique précéramique ancien chypriote (fin 9e, début 8e millénaires cal. BC), apparenté au PPNB ancien/moyen du Levant nord. Comptes Rendus Acad. Sci. Ser. IIA Earth Planet. Sci. 2000, 330, 75-82. [CrossRef]

70. Moutsiou, T.; Kassianidou, V. Geochemical characterisation of carnelian beads from Aceramic Neolithic Cyprus using portable X-ray Fluorescence Spectrometry (pXRF). J. Archaeol. Sci. Rep. 2019, 25, 257-265. [CrossRef]

71. Wadsworth, F.B.; Heap, M.J.; Dingwell, D.B. Friendly fire: Engineering a fort wall in the Iron Age. J. Archaeol. Sci. 2016, 67, 7-13. [CrossRef]

72. Berrocal-Rangel, L.; García, R.; Ruano, L.; Vigil de la Villa Mencía, R. Vitrified Walls in the Iron Age of Western Iberia: New Research from an Archaeometric Perspective. Eur. J. Archaeol. 2019, 22, 185-209. [CrossRef]

73. McCloy, J.S.; Marcial, J.; Clarke, J.S.; Ahmadzadeh, M.; Wolff, J.A.; Vicenzi, E.P.; Bollinger, D.L.; Ogenhall, E.; Englund, M.; Pearce, C.I.; et al. Reproduction of melting behavior for vitrified hillforts based on amphibolite, granite, and basalt lithologies. Sci. Rep. 2021, 11, 1272. [CrossRef] [PubMed] 
74. Freire-Lista, D.M.; Fort, R. Exfoliation microcracks in building granite. Implications for anisotropy. Eng. Geol. 2017, 220 , 85-93. [CrossRef]

75. Christison, D. The Prehistoric Forts of Peeblesshire. With Plans and Sketches. Proc. Soc. Soc. Antiqu. Scotl. 1887, $21,13-82$. Available online: http://journals.socantscot.org/index.php/psas/article/view/6225 (accessed on 3 July 2021).

76. Camino Mayor, J. Las murallas compartimentadas en los castros de Asturias: Bases para un debate. Arch. Español Arqueol. 2000, 73, 27-42. [CrossRef]

77. Zhang, S.; Yang, Y.; Storozum, M.J.; Li, H.; Cui, Y.; Dong, G. Copper smelting and sediment pollution in Bronze Age China: A case study in the Hexi corridor, Northwest China. Catena 2017, 156, 92-101. [CrossRef]

78. Sanjurjo-Sánchez, J.; Montero Fenollós, J.L. Chronology during the Bronze Age in the archaeological site Tell Qubr Abu al-`Atiq, Syria. J. Archaeol. Sci. 2012, 39, 163-174. [CrossRef]

79. Sanjurjo-Sánchez, J.; Montero Fenollós, J.L.; Prudêncio, M.I.; Barrientos, V.; Marques, R.; Dias, M.I. Geochemical study of beveled rim bowls from the Middle Syrian Euphrates sites. J. Archaeol. Sci. Rep. 2016, 7, 808-818. [CrossRef]

80. Frahm, E. Buying local or ancient outsourcing? Locating production of prismatic obsidian blades in Bronze-Age Northern Mesopotamia. J. Archaeol. Sci. 2014, 41, 605-621. [CrossRef]

81. Lewis, M.P.; Quinn, P.S.; Carter, R. Uruk expansion or integrated development? A petrographic and geochemical perspective from Gurga Chiya, Iraqi Kurdistan. J. Archaeol. Sci. Rep. 2020, 33, 102516. [CrossRef]

82. Britishmuseum. Available online: https://www.britishmuseum.org/collection/object/W_1892-1213-9 (accessed on 25 June 2021).

83. Kohlmeyer, K. The Temple of the Storm God in Aleppo during the Late Bronze and Early Iron Ages. Near East. Archaeol. 2009, 72, 4. [CrossRef]

84. Emami, M.; Razani, M.; Alidadi Soleimani, A.N.; Madjidzadeh, Y. New insights into the characterization and provenance of chlorite objects from the Jiroft civilization in Iran. J. Archaeol. Sci. Rep. 2017, 16, 194-204. [CrossRef]

85. Rabbani, M.A. The typology, production and adornment of Gandharan beads during the mid-3rd century BCE-1st century CE: Preliminary results from Barikot, Swat, Pakistan. Archaeol. Res. Asia 2020, 24, 100228. [CrossRef]

86. Mutin, B.; Garazhian, O.; Shakooie, M. The Neolithic regional settlement of Darestan, Southern Lut Desert, Iran. Archaeol. Res. Asia 2020, 24, 100230. [CrossRef]

87. Fagan, B.M. A Little History of Archaeology; Yale Univerity Press: New Haven, CT, USA, 2018.

88. Ismael, I.S.; Hassan, M.S. Characterization of some Egyptian serpentinites used as ornamental stones. Chin. J. Geochem. 2008, 27, 140-149. [CrossRef]

89. Harrell, J.A.; Storemyr, P. Ancient Egyptian quarries-An illustrated overview. In QuarryScapes: Ancient Stone Quarry Landscapes in the Eastern Mediterranean; Abu-Jaber, N., Bloxam, E.G., Degryse, P., Heldal, T., Eds.; Geological Survey of Norway: Trondheim, Norway, 2009; Volume 12, pp. 7-50. 20p, Available online: https:/ /www.ngu.no/upload/Publikasjoner/Special\%20publication/ SP12_s7-50.pdf (accessed on 3 July 2021).

90. Harrell, J.A. Amarna gypsite: A new source of gypsum for ancient Egypt. J. Archaeol. Sci. Rep. 2017, 11, 536-545. [CrossRef]

91. Gouda Temraz, M.; Khallaf, M.K. Weathering behavior investigations and treatment of Kom Ombo temple sandstone, EgyptBased on their sedimentological and petrogaphical information. J. Afr. Earth Sci. 2016, 113, 194-204. [CrossRef]

92. Klemm, D.D.; Klemm, R. The building stones of ancient Egypt-A gift of its geology. J. Afr. Earth Sci. 2001, 33, 631-642. [CrossRef]

93. Park, H.D.; Shin, G.H. Geotechnical and geological properties of Mokattam limestones: Implications for conservation strategies for ancient Egyptian stone monuments. Eng. Geol. 2009, 104, 190-199. [CrossRef]

94. Braekmans, D.; Boschloos, V.; Hameeuw, H.; Van der Perre, A. Tracing the provenance of unfired ancient Egyptian clay figurines from Saqqara through non-destructive X-ray fluorescence spectrometry. Microchem. J. 2019, 145, 1207-1217. [CrossRef]

95. Madkour, F.S.; Khallaf, M.K. Degradation Processes of Egyptian Faience Tiles in the Step Pyramid at Saqqara. Procedia Soc. Behav. Sci. 2012, 68, 63-76. [CrossRef]

96. Flouda, G.; Philippidis, A.; Mikallou, A.; Anglos, D. Materials analyses of stone artifacts from the EBA to MBA Minoan Tholos tomb P at Porti, Greece (Crete), by means of Raman spectroscopy: Results and a critical assessment of the method. J. Archaeol. Sci. Rep. 2020, 32, 102436. [CrossRef]

97. Brilli, M.; Cavazzini, G.; Turi, B. New data of $87 \mathrm{Sr} / 86 \mathrm{Sr}$ ratio in classical marble: An initial database for marble provenance determination. J. Archaeol. Sci. 2005, 32, 1543-1551. [CrossRef]

98. Melfos, V. Mineralogical and Stable Isotopic Study of Ancient White Marble Quarries in Larisa, Thessaly, Greece. Bull. Geol. Soc. Greece 2004, 36, 1164-1172. [CrossRef]

99. Klein-Franke, F. The Knowledge of Aristotle's Lapidary during the Latin Middle Ages. Ambix 1970, 17, 137-142. [CrossRef]

100. Caley, E.R.; Richards, J.F.C. Theophrastus on Stones: Introduction, Greek Text, English Translation, and Commentary; Ohio State University: Columbus, OH, USA, 1956; p. 238.

101. Theoulakis, P.; Bardanis, M. The stone of Piraeus at the monuments of the Acropolis of Athens. In Proceedings of the 9th International Congress on Deterioration and Conservation of Stone Venice, Venice, Italy, 19-24 June 2000; pp. $255-263$.

102. Vaccari, E. Judging by Color in the Early History of Geology and Paleontology. Palaeogeogr. Palaeoclimatol. Palaeoecol. 2012, 367, 147-152. [CrossRef]

103. Piovesan, R.; Maritan, L.; Meneghin, G.; Previato, C.; Baklouti, S.; Sassi, R.; Mazzoli, C. Stones of the façade of the Sarno Baths, Pompeii: A mindful construction choice. J. Cult. Herit. 2019, 40, 255-264. [CrossRef] 
104. Maxfield, V.; Peacock, D. The Roman Imperial Quarries-Survey and Excavation at Mons Porphyrites 1994-1998; Topography and Quarries, Egypt Exploration Society: London, UK, 2001; Volume 1, p. 339.

105. Germinario, L.; Zara, A.; Maritan, L.; Bonetto, J.; Hanchar, J.M.; Sassi, R.; Mazzoli, C. Tracking trachyte on the Roman routes: Provenance study of Roman infrastructure and insights into ancient trades in northern Italy. Geoarchaeology 2018, 33, 417-429. [CrossRef]

106. Vidal Álvarez, S.; García-Entero, V. Marmora from the Roman Site of Carranque (Toledo, Spain). Marmora Int. J. Archaeol. Hist. Archaeom. Marbles Stone 2007, 3, 1-17. [CrossRef]

107. Kramar, S.; Zalar, V.; Urosevic, M.; Körner, W.; Mauko, A.; Mirtič, B.; Lux, J.; Mladenović, A. Mineralogical and microstructural studies of mortars from the bath complex of the Roman villa rustica near Mošnje (Slovenia). Mater. Charact. 2011, 62, $1042-1057$. [CrossRef]

108. Capedri, S.; Venturelli, G.; De Maria, S.; Mantovani Uguzzonia, M.P.; Pancotti, G. Characterisation and provenance of stones used in the mosaics of the domus dei Coiedii at Roman Suasa (Ancona, Italy). J. Cult. Herit. 2001, 2, 7-22. [CrossRef]

109. Antonelli, F.; Lazzarini, L. Mediterranean trade of the most widespread Roman volcanic millstones from Italy and petrochemical markers of their raw materials. J. Archaeol. Sci. 2010, 37, 2081-2092. [CrossRef]

110. Fabbri, S.; Chiarini, V.; Ercolani, M.; Sansavini, G.; Santagata, T.; De Waele, J. Terrestrial laser scanning, geomorphology and archaeology of a Roman gypsum quarry (Vena del Gesso Romagnola area, Northern Apennines, Italy). J. Archaeol. Sci. Rep. 2021, 36, 102810. [CrossRef]

111. Ontiveros-Ortega, E.; Beltrán Fortes, J.; Loza Azuaga, M.L. Mineralogical petrographic and geochemical characterization of marmora from the Roman quarries of Cabra (Córdoba) and Antequera (Málaga), External Sector Areas of the Betic Chain, Spain. J. Archaeol. Sci. Rep. 2019, 27, 101956. [CrossRef]

112. Green, C.; Jones, M.; Lovell, B.; Tubb, J. Discovery of a second Roman quarry in Hertfordshire for manufacture of querns from Paleogene Hertfordshire Puddingstone siliceous concretions. Proc. Geol. Assoc. 2016, 127, 359-362. [CrossRef]

113. Antonioli, F.; Mourtzas, N.; Anzidei, M.; Auriemma, R.; Galili, E.; Kolaiti, E.; Lo Presti, V.; Mastronuzzi, G.; Scicchitano, G.; Spampinato, C.; et al. Millstone quarries along the Mediterranean coast: Chronology, morphological variability and relationships with past sea levels. Quat. Int. 2017, 439, 102-116. [CrossRef]

114. Wirsching, A. How the obelisks reached Rome: Evidence of Roman double-ships. Int. J. Naut. Archaeol. 2000, $29,273-283$. [CrossRef]

115. Hällström, J.; Barup, K.; Grönlund, R.; Johansson, A.; Svanberg, S.; Palombi, L.; Lognoli, D.; Raimondi, V.; Cecchi, G.; Conti, C. Documentation of soiled and biodeteriorated façades: A case study on the Coliseum, Rome, using hyperspectral imaging fluorescence lidars. J. Cult. Herit. 2009, 10, 106-115. [CrossRef]

116. Coli, M.; Rosati, G.; Pini, G.; Baldi, M. The Roman quarries at Antinoopolis (Egypt): Development and techniques. J. Archaeol. Sci. 2011, 38, 2696-2707. [CrossRef]

117. Grewe, K. Als das Wasser laufen lernte. Die Eifel-ein technikgeschichtliches Freilichtmuseum. In Endlich Eifel-Wasser der Eifel; Eifelbildverlag: Daun, Germany, 2021; pp. 24-27.

118. Columbu, S.; Lisci, C.; Sitzia, F.; Lorenzetti, G.; Lezzerini, M.; Pagnotta, S.; Raneri, S.; Legnaioli, S.; Palleschi, V.; Gallello, G.; et al. Mineralogical, Petrographic and Physical-Mechanical Study of Roman Construction Materials from the Maritime Theatre of Hadrian's Villa (Rome, Italy). Measurement 2018, 127, 264-276. [CrossRef]

119. Oliver Domingo, J.L. Los Diez Libros de Arquitectura. Traducción de la Obra Original de Marco Vitruvio Polion; Alianza Editorial: Madrid, Spain, 1997; p. 400.

120. Carey, S. Pliny's Catalogue of Culture: Art and Empire in the Natural History; Oxford University Press: Oxford, UK, 2003.

121. Seabra, L.; Tereso, J.; Bettencourt, A.M.S.; Dinis, A. Crop diversity and storage structures in the settlement of Crastoeiro (Northwest Iberia): New approaches. Trab. Prehist. 2018, 75, 361-378. [CrossRef]

122. Nicoll, K.; Zerboni, A. Is the past key to the present? Observations of cultural continuity and resilience reconstructed from geoarchaeological records. Quat. Int. 2020, 545, 119-127. [CrossRef]

123. Sanjurjo-Sánchez, J.; Arce Chamorro, C.; Alves, C.; Sánchez-Pardo, J.C.; Blanco-Rotea, R.; Costa-García, J.M. Using in situ gamma ray spectrometry (GRS) exploration of buried archaeological structures: A case study from NW Spain. J. Cult. Herit. 2018, 34, 247-254. [CrossRef]

124. Pini, R.; Siano, S.; Salimbeni, R.; Piazza, V.; Giamello, M.; Sabatini, G.; Bevilacqua, F. Application of a new laser cleaning procedure to the mausoleum of Theodoric. J. Cult. Herit. 2000, 1, 93-97. [CrossRef]

125. Sanjurjo-Sánchez, J.; Blanco-Rotea, R.; Sánchez-Pardo, J.C. An Interdisciplinary Study of Early Mediaeval Churches in NorthWestern Spain (Galicia). Heritage 2019, 2, 599-610. [CrossRef]

126. Taranto, M.; Barba, L.; Blancas, J.; Bloise, A.; Cappa, M.; Chiaravalloti, F.; Crisci, G.M.; Cura, M.; De Angelis, D.; De Luca, R.; et al. The bricks of Hagia Sophia (Istanbul, Turkey): A new hypothesis to explain their compositional difference. J. Cult. Herit. 2019, 38, 136-146. [CrossRef]

127. Çelik, M.Y.; Sert, M. The importance of "Pavonazzetto marble" (Docimium-Phrygia/Iscehisar-Turkey) since ancient times and its properties as a global heritage stone resource. Environ. Earth Sci. 2020, 79, 201. [CrossRef]

128. Abu El-Enen, M.M.; Lorenz, J.; Ali, K.A.; von Seckendorff, V.; Okrusch, M.; Schussler, U.; Bratz, H.; Schmitt, R.-T. A new look on Imperial Porphyry: A famous ancient dimension stone from the Eastern Desert of Egypt—petrogenesis and cultural relevance. Int. J. Earth Sci. 2018, 107, 2393-2408. [CrossRef] 
129. Melfos, V. Green Thessalian Stone: The Byzantine Quarries and the Use of a Unique Architectural Material from the Larisa Area, Greece. Petrographic and Geochemical Characterization. Oxf. J. Archaeol. 2008, 27, 387-405. [CrossRef]

130. Al-Bashaireh, K. Ancient white marble trade and its provenance determination. J. Archaeol. Sci. Rep. 2021, 35, 102777. [CrossRef]

131. Attanasio, D.; Brilli, M.; Bruno, M. Properties and identification of marble from Proconnesos (Marmara Island, Turkey): A New Database Including Isotopic, EPR and Petrographic Data. Archaeometry 2008, 50, 747-774. [CrossRef]

132. Moropoulou, A.; Christaras, B.; Lavas, G.; Penelis, G.; Zias, N.; Biscontin, G.; Kolliasf, E.; Paisios, A.; Theoulakis, P.; Bisbikou, K.; et al. Weathering Phenomena on the Hagia Sophia Basilica Konstantinople. WIT Trans. Built Environ. 1993, 3, 923-942. [CrossRef]

133. Mensing, S.; Tunno, I.; Cifani, G.; Passigli, S.; Noble, P.; Archer, C.; Piovesan, G. Human and climatically induced environmental change in the Mediterranean during the Medieval Climate Anomaly and Little Ice Age: A case from central Italy. Anthropocene 2016, 15, 49-59. [CrossRef]

134. Sánchez-Pardo, J.; Blanco-Rotea, R.; Sanjurjo-Sánchez, J. The church of Santa Comba de Bande and early medieval Iberian architecture: New chronological results. Antiquity 2017, 91, 1011-1026. [CrossRef]

135. Morris, C.D. Viking Orkney: A survey. In The Prehistory of Orkney; Renfrew, C., Ed.; Edinburgh University Press: Edinburgh, UK, 1985.

136. Polovodova, I.; Nordberg, K.; Filipsson, H.L. The benthic foraminiferal record of the Medieval Warm Period and the recent warming in the Gullmar Fjord, Swedish west coast. Mar. Micropaleontol. 2011, 81, 95-106. [CrossRef]

137. Ježek, M. Touchstones of archaeology. J. Anthropol. Archaeol. 2013, 32, 713-731. [CrossRef]

138. Ježek, M.; Hansen, S.C.J. Symbols missing a cause: The testimony of touchstones from Viking Age Iceland. Archaeol. Anthropol. Sci. 2019, 11, 3423-3434. [CrossRef]

139. Sanjurjo-Sánchez, J.; Pérez Mato, M. Delimiting the urban growth of Santiago de Compostela (NW Spain) by OSL dating of medieval anthropogenic sediments. Mediterr. Archaeol. Archaeom. 2013, 13, 163-173.

140. Rivas, T.; Pozo-Antonio, J.S.; Ramil, A. López; A.J. Influence of the weathering rate on the response of granite to nanosecond UV laser irradiation. Sci. Total Environ. 2020, 706, 135999. [CrossRef]

141. Buringh, E.; Campbell, B.M.S.; Rijpma, A.; van Zanden, J.L. Church building and the economy during Europe's 'Age of the Cathedrals', 700-1500 CE. Explor. Econ. Hist. 2020, 76, 101316. [CrossRef]

142. Coccia, S.; Como, M.; Di Carlo, F. Wind strength of Gothic Cathedrals. Eng. Fail. Anal. 2015, 55, 1-25. [CrossRef]

143. Ruffolo, S.A.; Comite, V.; La Russa, M.F.; Belfiore, C.M.; Barca, D.; Bonazza, A.; Crisci, G.M.; Pezzino, A.; Sabbioni, C. An analysis of the black crusts from the Seville Cathedral: A challenge to deepen the understanding of the relationships among microstructure, microchemical features and pollution sources. Sci. Total Environ. 2015, 502, 157-166. [CrossRef] [PubMed]

144. Shuriye, A.O.; Danzomo, B.A. The Contribution of Al-Khazini in the Development of Hydrostatic Balance and Its Functionality, in Contributions of Early Muslim Scientists to Engineering Sciences and Related Studies; International Islamic University Malaysia (IIUM) Press: Kuala Lumpur, Malaysia, 2011; p. 170.

145. Shafik Ramzy, N. Concept cathedral and "squaring the circle": Interpreting the Gothic cathedral of Notre Dame de Paris as a standing hymn. Front. Archit. Res. 2021, 10, 369-393. [CrossRef]

146. Kozak-Holland, M.; Procter, C. Florence Duomo project (1420-1436): Learning best project management practice from history. Int. J. Proj. Manag. 2014, 32, 242-255. [CrossRef]

147. Foraboschi, P. The central role played by structural design in enabling the construction of buildings that advanced and revolutionized architecture. Constr. Build. Mater. 2016, 114, 956-976. [CrossRef]

148. Pascale, G.; Lolli, A. Crack assessment in marble sculptures using ultrasonic measurements: Laboratory tests and application on the statue of David by Michelangelo. J. Cult. Herit. 2015, 16, 813-821. [CrossRef]

149. Germinario, L.; Török, Á. Surface Weathering of Tuffs: Compositional and Microstructural Changes in the Building Stones of the Medieval Castles of Hungary. Minerals 2020, 10, 376. [CrossRef]

150. Suchodoletz, H.; Gärtner, A.; Zielhofer, C.; Faust, D. Eemian and post-Eemian fluvial dynamics in the Lesser Caucasus. Quat. Sci. Rev. 2018, 191, 189-203. [CrossRef]

151. Mariani, G.S.; Compostella, C.; Trombino, L. Complex climate-induced changes in soil development as markers for the Little Ice Age in the Northern Apennines (Italy). Catena 2019, 181, 104074. [CrossRef]

152. Galindo Díaz, J.A. El Conocimiento Constructivo de los Ingenieros Militares Españoles del Siglo XVIII. Un Estudio Sobre la Formalización del Saber Técnico a Través de los Tratados de Arquitectura Militar. Ph.D. Thesis, UPC, Departament de Construccions Arquitectòniques I, Barcelona, Spain, 1996. Available online: http://hdl.handle.net/2117/93417 (accessed on 3 July 2021).

153. Freire-Lista, D.M.; Fort, R.; Varas-Muriel, M.J. Nomination of Zarzalejo granite, a Spanish heritage building stone, as a “Global Heritage Stone Resource". Energy Procedia 2015, 76, 642-651. [CrossRef]

154. Sánchez Martínez, F.V.; Arenas Reina, J.M.; Recio Díaz, M.M.; Horcajo de Frutos, R. Marble cutting processing used in 16th century for building the "El Escorial" monastery altarpiece. Procedia Manuf. 2017, 13, 1381-1388. [CrossRef]

155. Leone, G.; De Vita, A.; Magnani, A.; Rossi, C. Characterization of archaeological mortars from Herculaneum. Thermochim. Acta 2016, 624, 86-94. [CrossRef]

156. Manrique-Ortega, M.D.; Casanova-González, E.; Mitrani, A.; González-Cruz, A.; Cuevas-García, M.; Ruvalcaba-Sil, J.L. Spectroscopic examination of Red Queen's funerary mask and her green stone offering from the Mayan site of Palenque, Mexico. Spectrochim. Acta Part A 2020, 234, 118205. [CrossRef] 
157. Freire-Lista, D.M.; Fort, R.; Varas-Muriel, M.J. Thermal shock-induced microcracking in building granite. Eng. Geol. 2016, 203, 83-93. [CrossRef]

158. Bromley, W. Several Years Travels Through Portugal, Spain, Italy, Germany, Prussia, Sweden, Denmark and the United Provinces. Performed by a Gentleman; Eighteenth-Century Britain: London, UK, 2018.

159. Álvarez de Colmenar, J. Les Delices de l'Espagne \& du Portugal: Où l'on Voit une Description Exacte des Antiquitez, des Provinieses; Chez Pierre Vander Aa: Leide, The Netherlands, 1707.

160. Carter, F. A Journey from Gibraltar to Malaga: With a View of that Garrison and Its Environs; A Particular Account of the Towns in the Hoya of Malaga and Thirteen Plates Engraved from Original Drawings, Taken in the Year. Nichols, F., Ed.; London, UK. 1772. Available online: http:/ / bdh-rd.bne.es/viewer.vm?id=0000000789 (accessed on 4 July 2021).

161. Twiss, R. Travels Through Portugal and Spain, in 1772 and 1773. with Copper-Plates, and an Appendix; Eighteenth-Century Britain: London, UK, 1775.

162. Swinburne, H. Travels Through Spain, in the Years 1775 and 1776: In Which Several Monuments of Roman and Moorish Architecture are Illustrated by Accurate Drawings Taken on the Spot; Elmsly, P., Ed.; TheClassics: London, UK, 2013; p. 427.

163. Margarot, M. Histoire ou Relation d'un Voyage qui a duré près de cinq ans; Pendant Lequel l'Auteur a Parcouru une Partie de l'Angleterre, la France, l'Espagne. Par Mr. Maurice Margarot, le Pêre; 1780; Eighteenth-Century Britain: London, UK, 2010.

164. Dillon, J.T. Travels Through Spain with a View to Illustrate the Natural History and Physical Geography of that Kingdom, in a Series of Interspersed with Historical Anecdotes; Balwin, R., Ed.; Pearson and Rollason: Birmingham, UK, 1780; p. 459.

165. Townsend, J. A Journey through Spain: In the Years 1786 and 1787 and Remarks in Passing through a Part of France; in Three Volumes. Dilly, C., Ed.; London, UK. 1791. Available online: http://www.bibliotecavirtualdeandalucia.es/catalogo/es/consulta/ registro.cmd?id=6069 (accessed on 4 July 2021).

166. De Langle, J.M.-J.F. Voyage en Espagne-Cinquieme Edition. Avec Figures et Carte Géographique; Lucet, J.J., Ed.; Chez Blanchon: Paris, France, $1796 ; 262 \mathrm{p}$.

167. Vaccari, E.; Wyse Jackson, P.N. The fossil fishes of Bolca and the travels in Italy of the Irish cleric George Graydon in 1791. Museol. Sci. 1995, 4, 57-81.

168. Hannibal, J.T.; Thomas, S.F.; Noll, M.G. Maximilian, Prince of Wied's Trip Along the Ohio \& Erie Canal in 1834: An Annotated New Translation. Ohio Hist. 2009, 116, 5-25.

169. Ponz, A. Viage de España, or Cartas, en que se da Noticia de las Cosas más Apreciables, y Dignas de Saberse que hay en Ella; Joaquin Ibarra: Madrid, Spain, 1781; p. 57.

170. Freire-Lista, D.M.; Fort, R. Historical Quarries, Decay and Petrophysical Properties of Carbonate Stones Used in the Historical Center of Madrid (Spain). AIMS Geosci. 2017, 3, 284-302. [CrossRef]

171. Carneiro, A.; Klemun, M. Instruments of science-Instruments of geology; Introduction to seeing and measuring, constructing and judging: Instruments in the history of the earth sciences. Centaurus 2011, 53, 77-85. [CrossRef]

172. Pococke, R. A Description of the East and Some Other Countries Volume 1. Printed for the Autor Richard Pococke. Bowyer, 1743. Egypt. p. 310. Available online: https://gallica.bnf.fr/ark:/12148/bpt6k850870q/f7.item (accessed on 3 July 2021).

173. Panckoucke. Description de l'Égypte. Taschen 1994. p. 1007. Available online: https://gallica.bnf.fr/ark:/12148/bpt6k27999f. texteImage (accessed on 4 July 2021).

174. Winckelmann, J.J. Gedanken über die Nachahmung der griechischen Werke in der Malerei und Bildhauerkunst. In Deutsche Literaturdenkmale des 18. und 19. Jahrhunderts in Neudr; De Gruyter: Berlin, Germany, 1885; Available online: https://www. degruyter.com/document/doi/10.1515/9783112367124/html (accessed on 3 July 2021). [CrossRef]

175. Liljegren, J.G.; Brunius, C.G. Nordiska Fornlemningar; Haeggström: Stockholm, Sweden, 1823.

176. Thomsen, C.J. Ledetraad til Nordisk Oldkyndighed. Møllers, S.L., Ed.; Bogtr. 1836. 100p. Available online: https: / /books.google.pt/books?id=IWTGAAAAIAAJ\&printsec=frontcover\&dq=Ledetraad+til+Nordisk+Oldkyndighed\&hl=es\& sa=X\&ved=2ahUKEwjWoKTD8LzvAhXrDWMBHaNUCSYQ6AEwAHoECAEQAg\#v=onepage\&q=Ledetraad\%20til\%20 NorNord\%20Oldkyndighed\&f=false (accessed on 3 July 2021).

177. Gardner Wilkinson, J. Manners and Customs of the Ancient Egyptians; Murray, J., Ed.; Cambridge University Press: London, UK, 1841; Available online: https://books.google.pt/books?id=Z3wOAAAAQAAJ\&printsec=frontcover\&dq=Manners+and+Customs+ of+the+Ancient+EgyEgypti\&hl=es\&sa=X\&ved=2ahUKEwjmuZD18bzvAhUoA2MBHSdHDmUQ6AEwAHoECAMQAg\#v= onepage\&q=Manners $\% 20$ and $\% 20$ Customs $\% 20$ of $\% 20$ the $\% 20$ Ancient $\% 20$ Egyptians $\& f=$ false (accessed on 3 July 2021).

178. Freire-Lista, D.M. Geotourism from Fuente de Cibeles of Madrid. History, Building Stones and Quarries. Cadernos do Laboratorio Xeolóxico de Laxe. Rev. Xeol. Galega Hercínico Penins. 2020, 42, 69-94. [CrossRef]

179. Villa-Amil y Castro, J. Antigüedades Prehistóricas y Célticas de Galicia. Freire, S., Ed.. 1873. Available online: https: / / books.google.pt/books?id=mHSvXAsL8FAC\&printsec=frontcover\&dq=Antig $\%$ C3\%BCedades+Prehist $\%$ C3 $\%$ B3ricas $+y+$ $\mathrm{C} \% \mathrm{C} 3 \%$ A9lticas+de+Galicia\&hl=es\&sa=X\&ved=2ahUKEwimjaTy8rzvAhWOoBQKHRaCCwoQ6AEwAHoECAEQAg\#v= onepone\&q=Antig $\%$ C3\%BCedades $\% 20$ Prehist $\%$ C3\%B3ricas $\% 20 y \% 20$ C $\% 3 \%$ A9lticas $\% 20 \mathrm{de} \% 20$ Galicia\&f=false (accessed on 3 July 2021). 
180. Lartet, E.; Christy, H. Reliquiae Aquitanicæ: Being Contributions to the Archæology and Palæontology of Perigord and the Adjoining Provinces of Southern France. Geol. Mag. 1866, 3, 76-77. Available online: https://books.google. pt/books?id=mvVmAAAAcAAJ\&printsec=frontcover\&dq=Reliquiae+Aquitanicae\&hl=es\&sa=X\&ved=2aahUKEwix8TY8 7zvAhUJoRQKHYxgA3MQ6AEwAHoECAEQAg\#v=onepage\&q=Reliquiae\%20Aquitanicae\&f=false (accessed on 3 July 2021). [CrossRef]

181. Déchelette, J. Manuel D’archéologie Préhistorique Celtique et Gallo-Romaine. Picard, A., Ed.; et fils. 1931. Available online: https://books.google.pt/books?id=Z-1XAAAAYAAJ\&q=Manuel+D\%27arch\%C3\%A9ologie+Pr\%C3\%A9historique, + Celtique+et+Gallo-Romaine\&dq=Manuel+D\%27arch\%C3\%A9ologie+Pr\%C3\%A9historique,+Celtique+et+Gallo-Romaine\& $\mathrm{hl}=\mathrm{es} \& \mathrm{sa}=X \& v e d=2 \mathrm{ahUKEwjVtauT9LzvAhVGCWMBHXTyAyAQ6AEwAHoECAAQAg}$ (accessed on 3 July 2021).

182. Hernández-Pacheco y Esteban, E. Las pinturas prehistóricas de Peña Tu (Asturias). In Comisión de Investigaciones Paleontológicas y Prehistóricas; Museo de Ciencias Naturales: Madrid, Spain, 1914; Volume 2, p. 35.

183. Hernández-Pacheco, E.E. El Museo de Ciencias Naturales y sus naturalistas de los siglos XVIII y XIX. In Publicaciones del Museo Nacional de Ciencias Naturales; Museo de Ciencias Naturales: Madrid, Spain, 1944; p. 81.

184. Hernández-Pacheco y Esteban, E. El solar en la Historia Hispana. Memorias de la Real Academia de Ciencias Exactas, Físicas y Naturales. In Serie de Ciencias Naturales; La Gaceta de Madrid: Madrid, Spain, 1952; Volume 15, p. 766.

185. Hernández-Pacheco y Esteban, E. Fisiografía del Solar Hispano. Memorias de la Real Academia de Ciencias Exactas, Físicas y Naturales. In Serie de Ciencias Naturales; La Gaceta de Madrid: Madrid, Spain, 1955; Volume 2, pp. 665-793.

186. Hernández-Pacheco y Esteban, E. Prehistoria del Solar Hispano. Memorias de la Real Academia de Ciencias Exactas, Físicas y Naturales, Serie de Ciencias Naturales; Real Academia de Ciencias Exactas, Físicas y Naturales: Madrid, Spain, 1959; p. 20.

187. Kölbl-Ebert, M. German petroleum geologists and World War II. J. Geol. Soc. Lond. Spec. Publ. 2018, 465, 391-407. [CrossRef]

188. Brandi, C. Teoría de la Restauración; Alianza Forma: Madrid, Spain, 1977; p. 149.

189. Freire-Lista, D.M.; Fort, R. Historical City Centres and Traditional Building Stones as Heritage: The Barrio de las Letras, Madrid (Spain). Geoheritage 2019, 11, 71-85. [CrossRef]

190. Bossi, L. Dizionario Portatile di Geologia, Litologia e Mineralogia; Giegler: Milano, Italy, 1819; p. 428.

191. Mitchell, J. A Dictionary of Chemistry, Mineralogy, and Geology: In Accordance with the Present State of those Sciences; Phillips: London, UK, 1823; p. 630.

192. De Thury, H. On the method proposed by Mr Brard for the immediate detection of stones unable to resist the action of frost. Ann. Chem. Phys. 1828, 38, 160-192.

193. Freire-Lista, D.M.; Fort, R.; Varas-Muriel, M.J. Freeze-thaw fracturing in building granites. Cold Reg. Sci. Technol. $2015,113,40-51$. [CrossRef]

194. Silva, B.; Ferreira Pinto, A.P.; Gomes, A.; Candeias, A. Admixtures potential role on the improvement of the freeze-thaw resistance of lime mortars. J. Build. Eng. 2021, 35, 101977. [CrossRef]

195. Gómez-Heras, M. Procesos y Formas de Deterioro Térmico en Piedra Natural del Patrimonio Arquitectónico. Ph.D. Thesis, Universidad Autónoma de Madrid, Madrid, Spain, 2006; p. 367.

196. Cooper, B. The Historic Use and Trading of Building Stone in South Australia, and Support for the Associated Industry. S. Aust. Geogr. J. 2011, 110, 5-29.

197. Fort, R.; Álvarez de Buergo, M.; Pérez-Monserrat, E.; Gómez-Heras, M.; Varas, M.J.; Freire-Lista, D.M. Evolution in the use of natural building stone in Madrid, Spain. Q. J. Eng. Geol. Hydrogeol. 2013, 46, 421-429. [CrossRef]

198. Marcos y Bausa, R. Manual del Albañil, 3rd ed.; Tipografia Editorial G. Estrada: Madrid, Spain, 1879; Available online: https: / / issuu.com/anarchitect/docs/name386364. (accessed on 3 July 2021).

199. Ansted, D.A. On the Decay and Preservation of Building Materials. J. Frankl. Inst. 1860, 70, 217-223. [CrossRef]

200. Hirschwald, J. Die Prufung der Naturlichen Bausteine auf ihre Wetterbestandigkeit; Ernst and Sohn: Berlin, Germany, 1908.

201. Howe, J.; Allen, J.A. The Geology of Building Stones; Arnold, E., Ed.; Edward Arnold: London, UK, $1910 ;$ p. 455.

202. Howe, J.; Allen, J.A. Stones and Quarries; Sir I. Pitman \& Sons, Ltd.: London, UK; New York, NY, USA, 1920.

203. Mahan, S.A.; Donlan, R.A.; Kardos, B. Luminiscence dating of anthopogenic features of the San Luis Valley, Colorado: From Stone huts to Stone walls. Quat. Int. 2015, 362, 50-62. [CrossRef]

204. Merrill, G.P. On the collection of Maine building stones in the United States National Museum. Proc. U. S. Natl. Mus. 1883, 6, 165-177. Available online: https:/ / repository.si.edu/bitstream/handle/10088/12560/USNMP-6_365_1883.pdf?sequence=1\& isAllowed $=y$ (accessed on 3 July 2021). [CrossRef]

205. Merrill, G.P. The Collection of Building and Ornamental Stones in the United States National Museum. 1889. Available online: https:// archive.org/details/collectionbuild00merrgoog/page/n4/mode/2up?ref=ol\&view=theater. (accessed on 3 July 2021).

206. Merrill, G.P. Stones for Building and Decoration; John Wiley and Sons: New York, NY, USA, 1891; p. 453.

207. Merrill, G.P. A treatise on Rocks, Rock-Weathering and Soils; Macmillan Co.: New York, NY, USA; London, UK, 1897 ; p. 411.

208. Julien, A.A. The durability of building stones in New York City and vicinity. In US Tenth Census, Building Stones and the Quarry Industry; Bureau of Census: Washington, DC, USA, 1884; pp. 364-393.

209. Baker, I.O. A Treatise on Masonry Construction; John Wiley and Sons: New York, NY, USA, 1890; p. 598.

210. Crosby, W.O.; Loughin, G.F. The Building Stones of Boston and Vicinity. Ph.D. Thesis, Massachusetts Institute of Technology, Cambridge, MA, USA, 1904. Volume 17. pp. 165-185. Available online: http://hdl.handle.net/1721.1/10043 (accessed on 3 July 2021). 
211. Dale, T.N. Chief commercial granites of Massachusetts, New Hampshire and Rhode Island. U. S. Geol. Survey. Bull. 1908, 354, 228.

212. Dale, T.N. The commercial granites of New England. U.S. Geol. Survey. Bul. 1923, 521. [CrossRef]

213. Eckel, E.C. Building Stones and Clays; John Wiley and Sons: New York, NY, USA, 1912; p. 465.

214. Kessler, D.W. Physical and chemical tests on the commercial marbles of the United States. J. Frankl. Inst. 1919, 187, 631-632. [CrossRef]

215. Currier, L.W. Geologic appraisal of dimension-stone deposits. U.S. Geol. Survey. Bull. 1960, 78. [CrossRef]

216. Taber, S. Frost heaving. J. Geol. 1929, 37, 428-461. [CrossRef]

217. Taber, S. The mechanics of frost heaving. J. Geol. 1930, 38, 303-317. [CrossRef]

218. Schaffer, R.J. Weathering of natural building stones. Department of Scientific and Industrial Research. In Building Research Special Report 18; H.M. Stationery Office: London, UK, 1932.

219. Winkler, E.M. Weathering rates as exemplified by Cleopatra's Needle in New York City. J. Geol. Educ. 1965, 13, 50-52. [CrossRef]

220. Winkler, E.M. Important agents of weathering for building and monumental stone. Eng. Geol. 1966, 1, 381-400. [CrossRef]

221. Winkler, E.M. The importance of air pollution in the corrosion of stone and metals. Eng. Geol. 1970, 4, 327-334. [CrossRef]

222. Winkler, E.M. Stone. Properties, Durability in Man's Environment; Springer: New York, NY, USA, 1973.

223. Hannibal, J.T. Downtown Cleveland Rocks: A Look at Rock Types Used for Buildings and Monuments in Downtown Cleveland; Wade Oval, University Circle: Cleveland, OH, USA, 1987.

224. Hannibal, J.T. Guide to Stones Used for Houses of Worship in Northeastern Ohio; Urban Center, Maxine Goodman Levin College of Urban Affairs, Cleveland State University: Cleveland, OH, USA, 1990.

225. Doehne, E.; Price, C.A. Stone Conservation: An Overview of Current Research, 2nd ed.; The Getty Conservation Institute: Los Angeles, CA, USA, 2010; p. 159.

226. Siegesmund, S.; Snethlage, R. Stone in Architecture Properties, Durability; Springer: Berlin, Germany, $2014 ;$ p. 347.

227. Zalooli, A.; Freire-Lista, D.M.; Khamehchiyan, M.; Reza Nikudel, M.; Fort, R.; Ghasemi, S. Ghaleh-khargushi rhyodacite and Gorid andesite from Iran, characterization, uses and durability. Environ. Earth Sci. 2018, 77, 315. [CrossRef]

228. Torabi-Kaveh, M.; Heidari, M.; Mohseni, H.; Ménendez, B. Role of petrography in durability of limestone used in construction of Persepolis complex subjected to artificial accelerated ageing tests. Environ. Earth Sci. 2019, 78, 1-18. [CrossRef]

229. Delgado Rodrigues, J.; Ferreira Pinto, A.P. Stone consolidation by biomineralisation. Contribution for a new conceptual and practical approach to consolidate soft decayed limestones. J. Cult. Herit. 2019, 39, 82-92. [CrossRef]

230. Godts, S.; Orr, S.A.; Desarnaud, J.; Steiger, M.; Wilhelm, K.; De Clercq, H.; Cnudde, V.; De Kock, T. NaCl-related weathering of stone: The importance of kinetics and salt mixtures in environmental risk assessment. Herit. Sci. 2021, 9, 44. [CrossRef]

231. Halbrucker, E.; Fiers, G.; Vandendriessche, H.; De Kock, T.; Cnudde, V.; Crombé, P. Burning flint: An experimental approach to study the effect of fire on flint tools. J. Archaeol. Sci. Rep. 2021, 36, 102854. [CrossRef]

232. Maritan, L.; Ganzarolli, G.; Antonelli, F.; Rigo, M.; Kapatza, A.; Bajnok, K.; Coletti, C.; Mazzoli, C.; Lazzarini, L.; Vedovetto, P.; et al. What kind of calcite? Disclosing the origin of sparry calcite temper in ancient ceramics. J. Archaeol. Sci. 2021, $129,105358$. [CrossRef]

233. Czinder, B.; Vásárhelyi, B.; Török, A. Long-term abrasion of rocks assessed by micro-Deval tests and estimation of the abrasion process of rock types based on strength parameters. Eng. Geol. 2021, 282, 105996. [CrossRef]

234. Přikryl, R.; Přikrylová, J.; Racek, M.; Weishauptova, Z.; Kreislova, K. Decay mechanism of indoor porous opuka stone: A case study from the main altar located in the St. Vitus Cathedral, Prague (Czech Republic). Environ. Earth Sci. 2017, 76, 290. [CrossRef]

235. Moropoulou, A.; Labropoulos, K.-C.; Delegou, E.T.; Karoglou, M.; Bakolas, A. Non-destructive techniques as a tool for the protection of built cultural heritage. Constr. Build. Mater. 2013, 48, 1222-1239. [CrossRef]

236. Freire-Lista, D.M.; Kahraman, G.; Carter, R. Multi-analysis Characterisation of a Vernacular House in Doha (Qatar). Minerals 2019, 4, 241. [CrossRef]

237. Freire-Lista, D.M.; Sousa, L.; Carter, R.; Al-Na'îmî, F. Petrographic and Petrophysical Characterisation of the Heritage Stones of Fuwairit Archaeological Site (NE Qatar) and their Historical Quarries: Implications for Heritage Conservation. Episodes 2021, 44, 43-58. [CrossRef]

238. Sileo, M.; Gizzi, F.T.; Donvito, A.; Lasaponara, R.; Fiore, F.; Masini, N. Multi-Scale Monitoring of Rupestrian Heritage: Methodological Approach and Application to a Case Study. Int. J. Archit. Herit. 2020, 1-16. [CrossRef]

239. Luo, L.; Wang, X.; Guo, H.; Lasaponara, R.; Zong, X.; Masini, N.; Wang, G.; Shi, P.; Khatteli, H.; Chen, F.; et al. Airborne and spaceborne remote sensing for archaeological and cultural heritage applications: A review of the century (1907-2017). Remote Sens. Environ. 2019, 232, 111280. [CrossRef]

240. Lapenna, V.; Soldovieri, F. Preface to the special issue on "integration of space and in-situ techniques: A new paradigm for the monitoring and surveillance". Remote Sens. Environ. 2021, 253, 112192. [CrossRef]

241. Chase, A.F.; Chase, D.Z.; Weishampel, J.F.; Drake, J.B.; Shrestha, R.L.; Slatton, K.C.; Awe, J.J.; Carter, W.E. Airborne LiDAR, archaeology, and the ancient Maya landscape at Caracol, Belize. J. Archaeol. Sci. 2011, 38, 387-398. [CrossRef]

242. Throsby, D. Investment in urban heritage conservation in developing countries: Concepts, methods and data. City Cult. Soc. 2016, 7, 81-86. [CrossRef]

243. Ergün Hatir, M.; İnce, I. Lithology mapping of stone heritage via state-of-the-art computer vision. J. Build. Eng. 2021, 34, 101921. [CrossRef] 
244. Dino, G.A.; Clemente, P.; Lasagna, M.; De Luca, D.A. Residual Sludge from Dimension Stones: Characterisation for their Exploitation in Civil and Environmental Applications. Energy Procedia 2013, 40, 507-514. [CrossRef]

245. Barroso, C.A.; Oliveira, D.V.; Ramos, L.F. Vernacular schist farm walls: Materials, construction techniques and sustainable rebuilding solutions. J. Build. Eng. 2018, 15, 334-352. [CrossRef]

246. Elert, K.; García Baños, E.; Ibañez Velasco, A.; Bel-Anzué, P. Traditional roofing with sandstone slabs: Implications for the safeguarding of vernacular architecture. J. Build. Eng. 2021, 33, 101857. [CrossRef]

247. Biró, A.; Hlavička, V.; Lublóy, E. Effect of fire-related temperatures on natural stones. Constr. Build. Mater. 2019, $212,92-101$. [CrossRef]

248. Fuentes, E.; Carballeira, R.; Prieto, B. Role of Exposure on the Microbial Consortiums on Historical Rural Granite Buildings. Appl. Sci. 2021, 11, 3786. [CrossRef]

249. Korobiichuk, V.; Shlapak, V.; Kryvoruchko, A.; Sobolevskyi, R. Analysis of Change in the Decorative Properties of Granites under Thermal Exposure. Mater. Sci. 2019, 2, 35-43. [CrossRef] 